\title{
Advance Data
}

\section{National Hospital Ambulatory Medical Care Survey: 2003 Emergency Department Summary}

\author{
by Linda F. McCaig, M.P.H., and Catharine W. Burt, Ed.D., Division of Health Care Statistics
}

\section{Abstract}

Objectives-This report describes ambulatory care visits to hospital emergency departments (EDs) in the United States. Statistics are presented on selected hospital, patient, and visit characteristics. Selected trends in ED utilization from 1993 to 2003 are also presented.

Methods - The data presented in this report were collected in the 2003 National Hospital Ambulatory Medical Care Survey (NHAMCS). NHAMCS is part of the ambulatory care component of the National Health Care Survey that measures health care utilization across various types of providers. NHAMCS is a national probability sample survey of visits to emergency and outpatient departments of non-Federal, short-stay, and general hospitals in the United States. Sample data are weighted to produce annual national estimates.

Results-During 2003, an estimated 113.9 million visits were made to hospital EDs, about 38.9 visits per 100 persons. From 1993 to 2003, increasing trends in ED utilization rates were observed for people ages 22-49 years, 50-64 years, and 65 years and over. Over 16 million patients arrived by ambulance (14.2 percent). At approximately 3 percent of visits, the patient had been seen in the ED within the past 72 hours. In 2003, abdominal pain, chest pain, fever, and cough were the leading patient complaints, accounting for nearly one-fifth of all visits. Acute upper respiratory infection was the leading illness-related diagnosis at ED visits. There were an estimated 40.2 million injury-related visits during 2003, or 14.1 visits per 100 persons. Diagnostic and screening services, procedures, and medications were provided at 89.7 percent, 45.4 percent, and 77.3 percent of visits, respectively. In 2003, approximately 14 percent of ED visits resulted in hospital admission. On average, patients spent 3.2 hours in the ED, of which 46.5 minutes were spent waiting to see a physician.

Keywords: emergency department visits $\bullet$ diagnoses $\bullet$ injury $\bullet$ medications $\bullet$ ICD-9-CM

\section{Introduction}

The National Hospital Ambulatory Medical Care Survey (NHAMCS) was inaugurated in 1992 to gather, analyze, and disseminate information about the health care provided by hospital emergency departments (EDs) and outpatient departments (OPDs). The NHAMCS is part of the ambulatory component of the National Health Care Survey, a family of surveys that measures health care utilization across various types of providers. More information about the National Health Care Survey can be found at the Centers for Disease Control and Prevention's National Center for Health Statistics (NCHS) Internet address: www.cdc.gov/ nchs.

Ambulatory medical care is the predominant method of providing health care services in the United States and occurs in a wide range of settings. The largest proportion of ambulatory care services occurs in physician offices (1). Since 1973, NCHS has collected data on patient visits to physicians' offices through the National Ambulatory

\section{Acknowledgments}

This report was prepared in the Division of Health Care Statistics. Dale Sanders in the Technical Services Branch generated the tables. Sarah Gousen in the Technical Services Branch contributed to the description of the sampling procedure and to the "Technical Notes." The report was edited by Klaudia M. Cox, typeset by Annette F. Holman, and the figures were produced by Jarmila Ogburn of the Office of Information Services, Information Design and Publishing Staff.

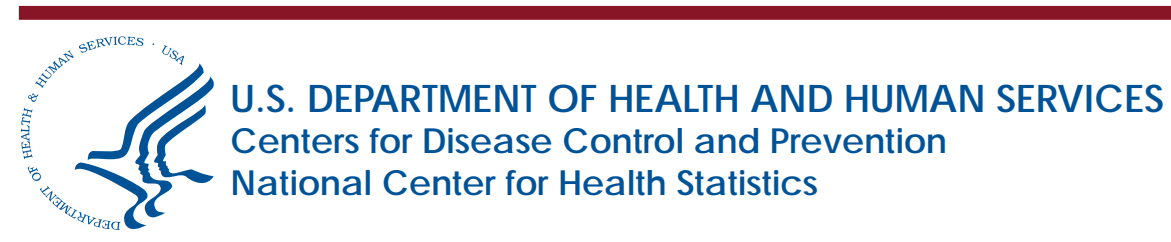


Medical Care Survey (NAMCS). However, visits to hospital EDs and OPDs, which represent a significant segment of ambulatory care visits, are not included in the NAMCS. EDs account for approximately 10 percent of all ambulatory medical care visits in the United States (1). Furthermore, hospital ambulatory patients are known to differ from office patients in certain demographic and medical characteristics (1).

The primary role of the ED is the treatment of seriously ill and injured patients. However, the ED provides a significant amount of unscheduled urgent care, often because there is inadequate capacity for this care in other parts of the health care system. The ED also serves as a referral site for other providers to evaluate and stabilize patients and admit them if necessary (2). ED administrators have reported being under increasing pressure to provide care for more patients, which has resulted in crowding (2). A recent report by the Government Accountability Office found three indicators that crowding is occurring: the number of hours that an ED is on ambulance diversion, the proportion of patients and length of time that patients "board" in the ED, and the proportion of patients who leave the ED before receiving a medical evaluation, usually because they tire of waiting. Another key indicator contributing to crowding is the inability to move patients out of EDs and into inpatient beds (3).

To reduce crowding, it is important for policymakers to understand the characteristics of frequent users. Most people do not use an ED in any given year. In 2003, 20.9 percent of children under 18 years of age and 20.0 percent of adults made one or more ED visits in the past 12 months and 7.0 percent of both children and adults made two or more visits (4). A Massachusetts study using data from fiscal year 2002 found that frequent ED users, defined as persons who made five or more visits in a year, represented 3.9 percent of all ED patients and accounted for 17.8 percent of all ED visits (5). Data from the Urban Institute's 1997 and 1999 National Surveys of America's Families showed that frequent ED users were more likely to be publicly insured, report worse health conditions, and have had more than three ambulatory care visits outside of the ED in the previous year. It appeared that frequent users did not substitute primary care visits for ED visits (6). To further examine the issues related to ED crowding and ambulance diversion in the United States, a supplement on this topic was added to the NHAMCS in 2003.

This report presents data from the 2003 NHAMCS on hospital, patient, and visit characteristics for EDs. New features in the 2003 report include data on selected ED utilization trends from 1993 to 2003, information on mode of arrival and waiting time (items absent from the survey since 2000), and more accurate estimates of proprietary hospitals as well as those not located in metropolitan statistical areas (MSAs). Finally, information on ED staffing and nursing triage levels is presented. Other Advance Data reports highlight visits to outpatient departments (7) and physician offices (8). More detailed information on the 1992-99 ED trend data has been published in a separate report (9).

\section{Data highlights}

\section{ED utilization}

- From 1993 to 2003, the number of ED visits increased from 90.3 million to 113.9 million visits annually (up 26 percent). This represents an average increase of more than 2 million visits per year. The number of hospital EDs in the United States decreased by about 12.3 percent during the same period.

- Approximately 58 percent of all hospital EDs were located in MSAs, but they represented 81.5 percent of the annual ED encounters.

- From 1993 to 2003, the overall ED utilization rate per population increased by 12 percent, from 35.5 percent to 39.9 percent.

- Infants under 12 months of age had a visit rate of 97.5 visits per 100 persons.

- Increasing trends in ED visit rates were found for persons ages 22-49 years (up by 19 percent), 50-64 years (up by 15 percent), and 65 years and over (up by 26 percent).

- Utilization rates were highest for Medicaid enrollees (81.0 visits per 100 persons) and lowest for patients with private insurance (21.5 visits per 100 persons).

- At 14.2 percent of visits, patients arrived at the ED by ambulance, representing over 16 million ambulance transports.

- The mean waiting time to see a physician was 46.5 minutes.

\section{Conditions seen}

- In nearly 1 out of 100 ED visits, the patient required immediate attention (e.g., unconscious or required resuscitation efforts).

- At about 3.3 million visits ( 2.8 percent), patients had been seen in the ED within the last 72 hours, and 5.5 percent of all visits were for followup of the same problem.

- Abdominal pain, chest pain, and fever were the most commonly recorded principal reasons for visit.

- The most frequently reported primary diagnoses were contusions, acute upper respiratory infections, abdominal pain, and chest pain. The leading diagnoses by age group were: infants and children (under 13 years of age)—acute respiratory infection, excluding pharyngitis; adolescents and young adults (13-21 years of age)—contusions; adults (22-49 years of age)—abdominal pain; middleaged persons (50-64 years of age) — chest pain; and seniors (65 years of age and over)-heart disease, excluding ischemic.

- Injury, poisoning, and adverse effects of medical treatment accounted for 35.3 percent of ED visits. Falls, being struck by or striking against, and motor vehicle traffic incidents were the leading causes of injuries presenting to the ED, accounting for about 41 percent of such visits.

- 1.7 million visits were for adverse effects of medical treatment.

\section{Services provided}

- Diagnostic and screening services and procedures were provided at 89.7 percent and 45.4 percent of visits, 
respectively. Imaging was provided at 42.9 percent of visits.

- Medications were provided at 77.3 percent of visits, and narcotic analgesics were the leading therapeutic class prescribed, at 14.1 percent of the medications mentioned.

- The duration for four-fifths of ED visits was between 1-6 hours. On average, patients spent 3.2 hours in the ED.

\section{Outcomes}

- About 15.8 million ED visits resulted in hospital admission, representing 13.9 percent of visits.

- After increasing for 10 years, the percentage of visits with no followup planned decreased to 6.3 percent.

- Over 2 million patients were transferred to other facilities (1.9 percent of visits), and 317,000 patients either were dead on arrival or died in the ED.

\section{ED-level estimates}

- Board-certified emergency medicine attending physicians and pediatric emergency medicine attending physicians were available at 63.5 percent and 18.1 percent of EDs, respectively.

\section{Methods}

The data presented in this report are from the 2003 NHAMCS, a national probability sample survey conducted by NCHS' Division of Health Care Statistics. The survey was conducted from December 30, 2002, through December 28, 2003.

The target universe of the NHAMCS is in-person visits made in the United States to EDs and OPDs of non-Federal, short-stay hospitals (hospitals with an average stay of less than 30 days) or those whose specialty is general (medical or surgical) or children's general. EDs that operate 24 hours a day are considered within the scope of the ED component; EDs that operate less than 24 hours are included in the OPD component of the NHAMCS. The hospital sampling frame consisted of hospitals listed in the 1991 Verispan Hospital Database (VHD) updated using the 2000 VHD to allow the inclusion of hospitals that opened or changed their eligibility status since the previous sample in 1991. The VHD was formerly known as the SMG Hospital Database. An additional sample of 66 hospitals (identified as located in non-MSAs or proprietary) was selected from the 2002 VHD to provide more reliable hospital estimates for these categories.

In 2003, a multistage probability sample was used to collect information on visits to emergency departments. The design included four stages for the majority of hospitals and three stages for an additional sample of 66 hospitals primarily located in rural areas. The NHAMCS has a four-stage design that involves a) geographic primary sampling units (PSUs), b) hospitals that have EDs or OPDs within PSUs, c) emergency service areas (ESAs) within EDs and clinics within OPDs, and d) patient visits within ESAs and clinics (10). The PSU sample consists of 112 PSUs that comprise a probability subsample of the PSUs used in the 1985-94 National Health Interview Survey. See the "Technical Notes" for more detail on the supplemental three-stage sample. Together, a sample of 546 hospitals was selected for the 2003 NHAMCS, of which 443 were in scope and had eligible EDs. A total of 475 ESAs from 406 EDs participated in the study. The overall unweighted two-stage sampling response rate was 85.3 percent, adjusted to exclude ESAs and EDs that participated at a minimal level (see "Technical Notes" for details). Hospital staff were asked to complete Patient Record forms (see figure I in the "Technical Notes") for a systematic random sample of patient visits occurring during a randomly assigned 4-week reporting period. The number of Patient Record forms completed for EDs was 40,253. Starting in 2001, sampling procedures were changed to target approximately 100 sample visits rather than 50 visits for each participating ED. The increased visit base led to increased precision for most visit estimates.
Because the estimates presented in this report are based on a sample rather than on the entire universe of ED visits, they are subject to sampling variability. The "Technical Notes" at the end of this report include an explanation of sampling errors with guidelines for judging the precision of the estimates. The standard errors reported here are calculated using Taylor approximations in SUDAAN, which take into account the complex sample design of the NHAMCS (11). Standard errors are provided in the text if the data are not presented in the tables. Data on selected ED utilization trends for 1993-2003 are presented. A weighted least-squares regression analysis was used to determine the significance of trends at the 0.05 level.

The U.S. Census Bureau was responsible for data collection. Data processing and medical coding were performed by Constella Group Inc., Durham, North Carolina. As part of the quality assurance procedure, a 10 percent quality control sample of survey records was independently keyed and coded. Coding error rates ranged between 0.0 and 0.8 percent for various survey items.

Several of the tables in this report present rates of ED visits per population. The population figures used in calculating these rates are based on Census Bureau monthly postcensal estimates of the civilian noninstitutional population of the United States as of July 1, 2003. These population estimates are based on postcensal estimates from Census 2000 and are available from the Census Bureau. For some rates, other denominators were used. See the "Technical Notes" for more detail on population figures and rate calculations. Estimates presented in the tables and figure for specific race categories reflect visits at which only a single race was reported. See the "Technical Notes" for more detail on race estimates.

In April 2003, the Privacy Rule of the Health Insurance Portability and Accountability Act (HIPAA) was implemented to establish minimum Federal standards for safeguarding the 

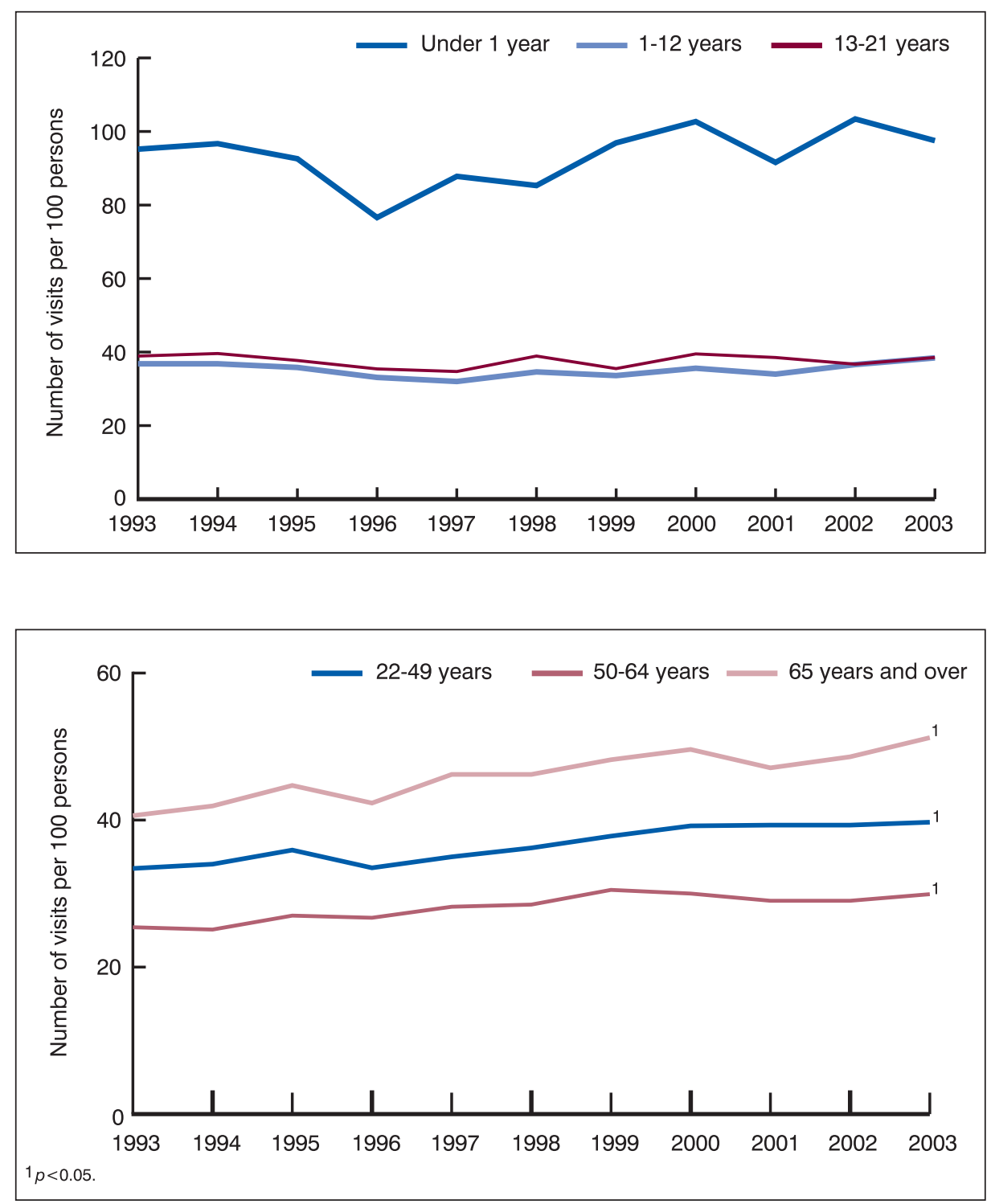

Figure 1. Trends in emergency department visit rates by age: United States, 1993-2003

privacy of individually identifiable health information. Therefore, the NHAMCS implemented additional data collection procedures to help providers assure patient confidentiality. See "Technical Notes" for more information.

\section{Results}

The ED utilization rate rose from 35.5 visits per 100 persons $(\mathrm{SE}=1.7)$ in 1993 to 39.9 visits per 100 persons in 2003 (up 12 percent) and was driven by increased use among adults (figure 1). The annual number of ED visits increased from 90.3 million to 113.9 million visits (up 26 percent). This represents an average increase of 2 million visits per year. On an average day in 2003, there were more than 312,000 visits to the ED. Because of hospitals either closing their EDs or going out of business, the number of operating EDs decreased by about 12.3 percent between 1993 and 2003 (12), resulting in a larger volume of visits to EDs remaining open. Increases in ED visits can lead to longer waiting times for nonurgent visits (13) and increased occurrence of ambulance diversion (14).

\section{Patient characteristics}

From 1993 to 2003, there was a 9-percent increase in the average age of patients seen in the ED, from 32.9 (SE $=0.5)$ to $35.9(\mathrm{SE}=0.5)$. ED visits by patient's age, sex, and race are shown in table 1 . Visit rates are presented by standard age categories in table 1 , showing variation across the age groups. In figure 1 , trends in visit rates by more specific age groups are displayed. From 1993 to 2003, increasing trends in visit rates were found for the following age groups: 65 years and over (up by 26 percent); 22-49 years (up by 19 percent); and 50-64 years (up by 15 percent) (figure 1 ). There was no difference in the visit rate by sex. About 48 percent of the visits being made by females were in the 25-64-year-old age group. The ED utilization rate for black or African American persons was 86 percent higher than for white persons (figure 2). Significant differences were observed by race in all age groups. ED visit rates for Asians were lower than for white persons or black or African American persons. Eleven percent $(\mathrm{SE}=$ 1.0) of visits were made by patients whose ethnicity was identified as Hispanic or Latino. Ethnicity was missing for 16.6 percent of records.

Patient residence-A relatively small proportion of the ED caseload (2.6 percent $[\mathrm{SE}=0.1]$ ) includes

\section{Differences in emergency department visit rates per 100 civilian persons by age and residence (noninstitutionalized compared with institutionalized)}

- Under 1 year (96.2 compared with estimate not reliable)

- 1-12 years (37.3 compared with estimate not reliable)

- 13-21 years (36.6 compared with 32.1)

- 22-49 years (37.5 compared with 24.8)

- 50-64 years (27.4 compared with 80.1)

- 65-74 years (33.6 compared with 164.0)

- 75-84 years (43.1 compared with 123.6)

- 85 years and over (55.4 compared with 124.0) 


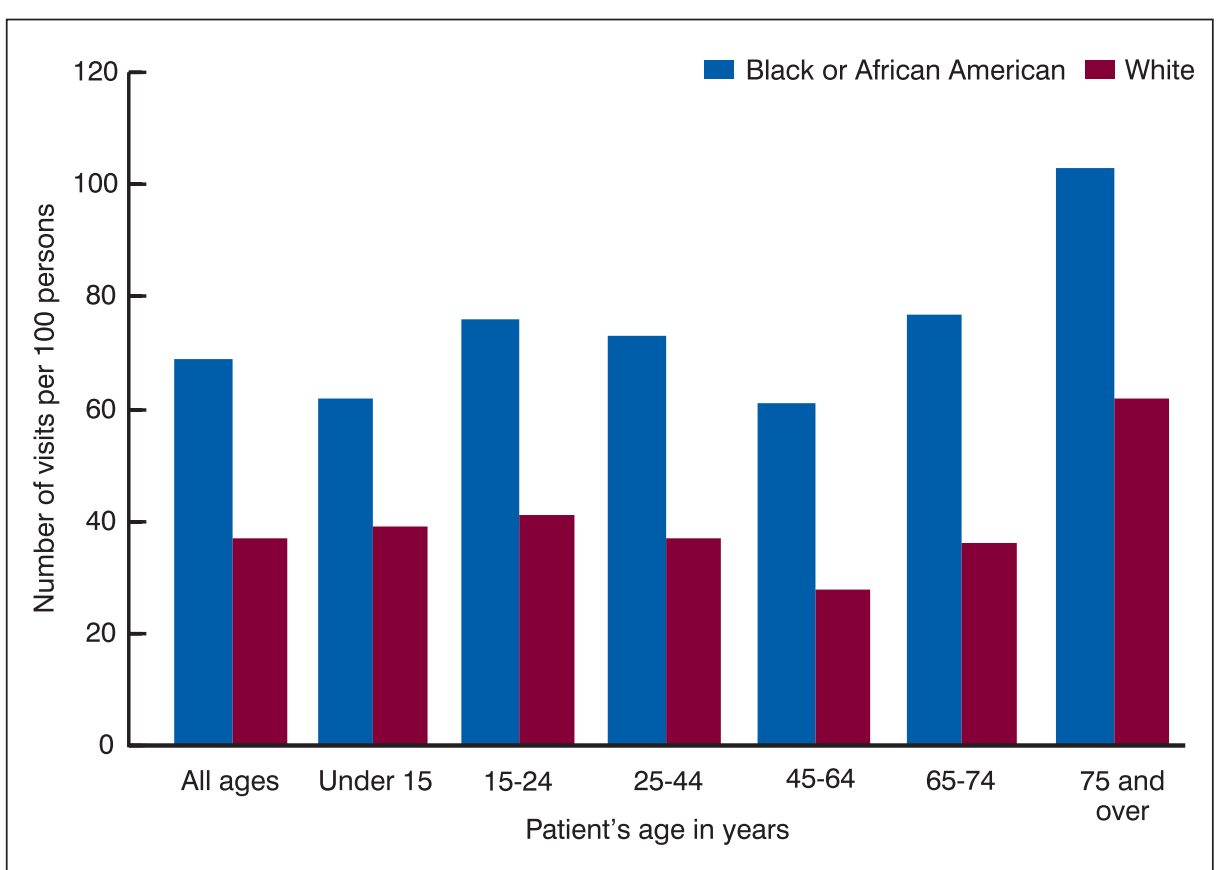

Figure 2. Annual rate of emergency department visits by patient's age and race: United States, 2003

persons living in institutions such as nursing homes or prisons; nevertheless, in 2003, they accounted for 2,913,000 ED visits. The average patient age for these visits was 71.1 years $(\mathrm{SE}=1.0)$ (data not shown). The text box shows visit rates for specific age groups by residence. For community-dwelling (noninstitutionalized) persons, infants had the highest visit rate (96.2 visits per 100 persons). For persons living in nursing homes or other institutions, persons 65-74 years of age had the highest rate (164.0 visits per 100 persons). Patient residence was missing for 5.3 percent of records, implying that the rates presented may be slight underestimates.

\section{Hospital characteristics}

Ownership-About 72 percent of ED visits were made to voluntary nonprofit hospitals (table 1). The percentage of visits made to non-Federal government (i.e., State, county, city) and proprietary hospitals was 18.5 percent and 9.3 percent, respectively.

Geographic location-Visit rates were higher in the Northeast (44.4 visits per 100 persons) and South (44.0 visits per 100 persons) than in the West (30.5 visits per 100 persons) (table 1). A higher proportion of ED visits occurred in the South (39.5 percent) than in the three other regions. The percentage of visits in the Midwest (22.1 percent) was greater than in the West (17.5 percent). About four out of five ED visits occurred in MSAs, but the population visit rates were not significantly different in MSAs and non-MSAs. See

"Technical Notes" for more detail about MSA visit rates.

\section{Visit characteristics}

Mode of arrival-At approximately 16 million visits (14.2 percent), the patient arrived at the ED by ambulance (either ground or air) (table 2). Over three-fourths of ED visits were made by patients who arrived at the ED by walking in, and 1.3 percent of arrivals were by public service (e.g., police or social services). For 5.4 percent of visits, mode of arrival was either recorded as "unknown" or left blank. Among persons 75 years of age and over, 40.9 percent arrived by ambulance. About 38 percent of visits $(\mathrm{SE}=1.2$ ) with ambulance recorded as the mode of arrival were made by persons 65 years of age and over (figure 3 ).

Primary expected source of payment-Private insurance was the most frequently recorded expected source of payment, accounting for 36.4 percent of ED visits (table 3 ). Medicaid or State Children's Health Insurance Program (SCHIP), Medicare, and self-payment (does not include patient copayments and deductibles) accounted for 21.4, 16.3, and 14.1 percent of visits, respectively. About 2 percent of ED visits cited Worker's Compensation as the primary expected source of payment. Payment mechanism varied by patient age, as shown in figure 4 . Private insurance was

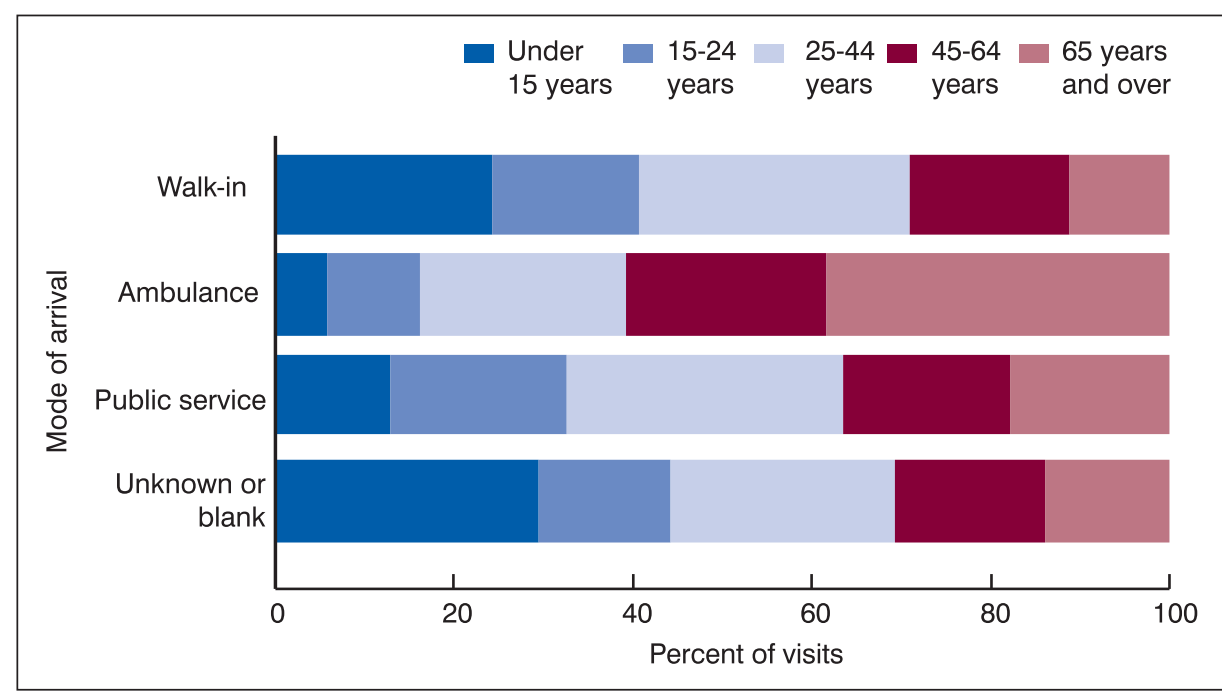

Figure 3. Percent distribution of emergency department visits by patient's age, according to mode of arrival: United States, 2003 


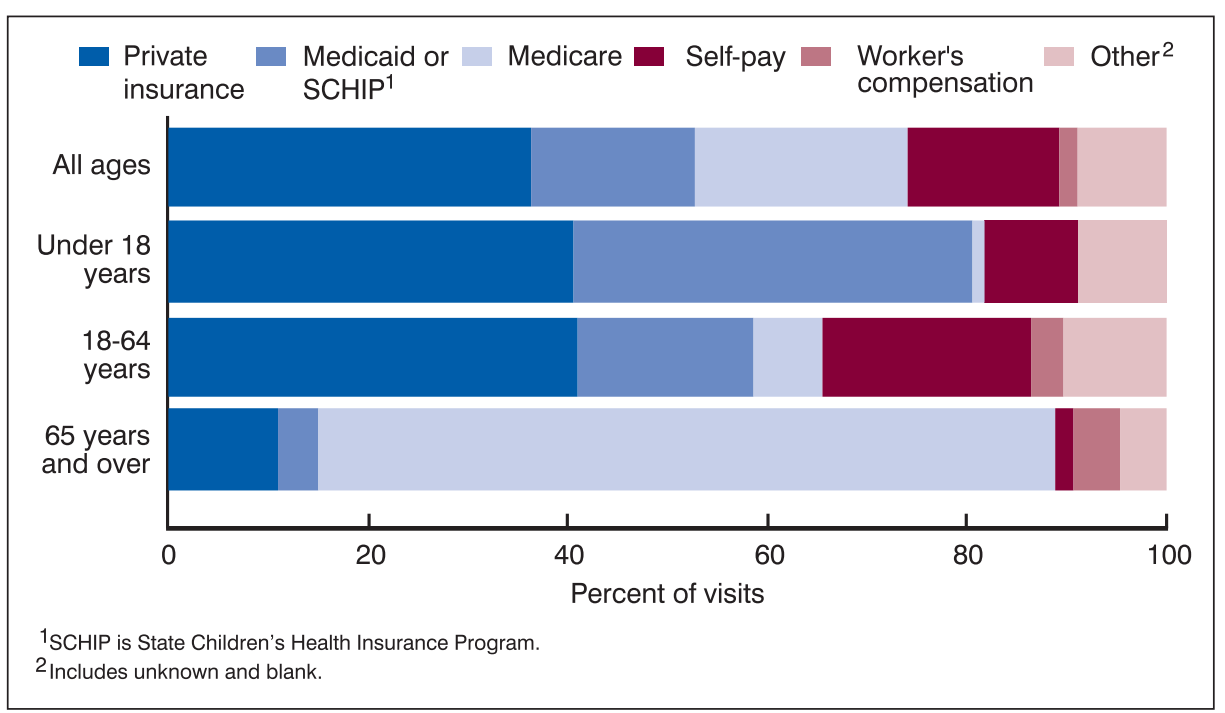

Figure 4. Percent distribution of emergency department visits by primary expected source of payment, according to patient's age: United States, 2003 the leading primary expected source of payment reported for persons under 65 years of age, and Medicare was most commonly recorded for persons aged 65 years and over. For 21.0 percent $(\mathrm{SE}=$ 1.0) of visits made by persons $18-64$ years of age, patients paid for ED services out of pocket. The visit rate for Medicaid patients (81.0 per 100 persons with Medicaid, $\mathrm{SE}=3.8$ ) was higher than those with Medicare (52.4 per 100 persons with Medicare, $\mathrm{SE}=2.5)$, no insurance (41.4 per 100 persons with no insurance, $\mathrm{SE}=2.4$ ), and private insurance (21.5 per 100 persons with private insurance, $\mathrm{SE}=1.0$ ) (data not shown).

\section{Immediacy with which patient} should be seen-The level of immediacy is assigned upon arrival at the ED by triage staff for EDs that conduct triage. The NHAMCS item

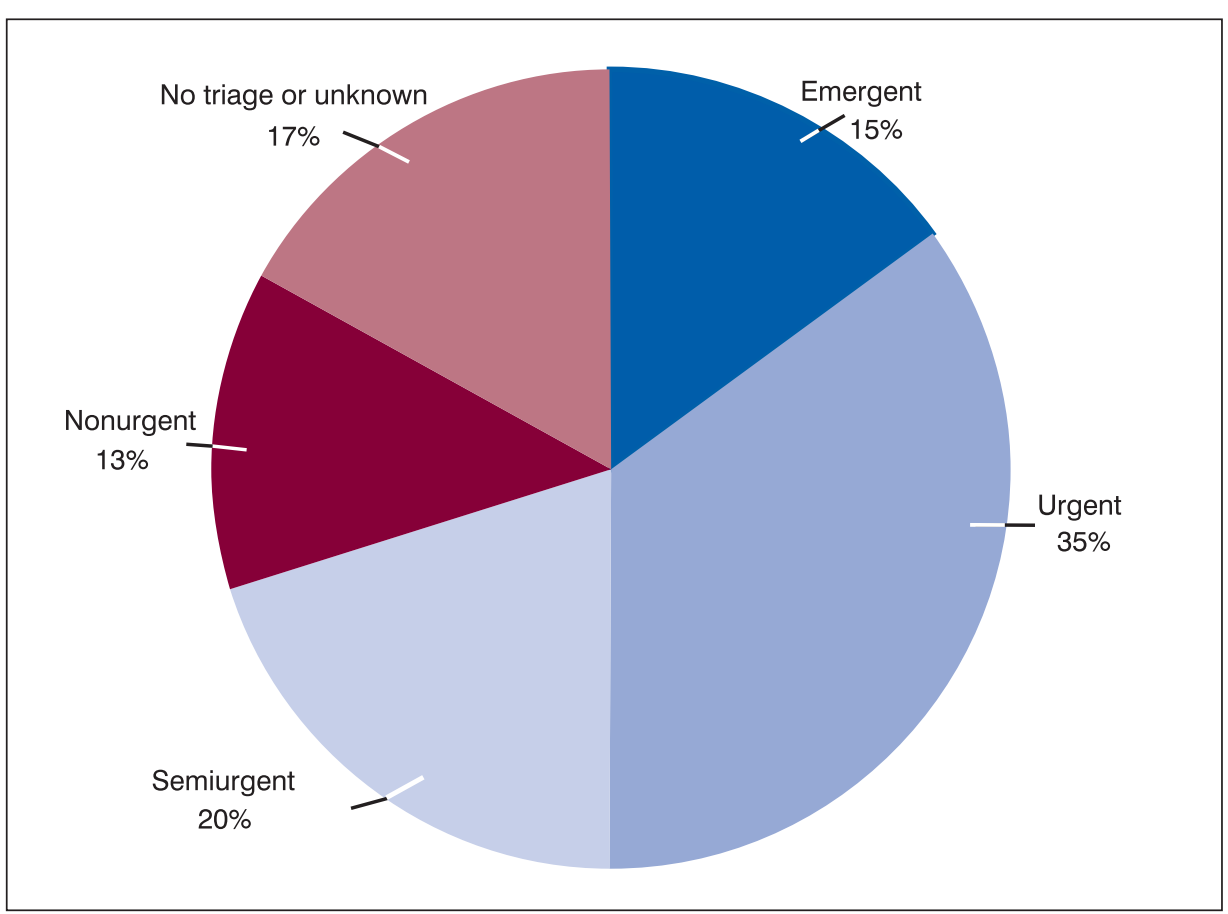

Figure 5. Percent distribution of emergency department visits by immediacy with which the patient should be seen: United States, 2003 categorized immediacy into five groups: emergent (less than 15 minutes), urgent (15-60 minutes), semiurgent (1-2 hours), nonurgent (2-24 hours), and "unknown or no triage." As shown in figure 5, 15 percent of ED visits were classified as emergent, 35 percent were urgent, 20 percent were semiurgent, 13 percent were nonurgent, and for 17 percent, the hospital staff recorded this item as "unknown or no triage." Data on urgency by patient and visit characteristics are presented in table 4. Persons 75 years of age and over had a higher proportion of emergent visits compared with all other age groups except persons 65-74 years of age. Together, emergent and urgent visits accounted for 50.4 percent of all ED visits. A higher proportion of Medicaid visits were triaged as nonurgent (15.5 percent) compared with Medicare visits (7.6 percent). There were $1,087,000$ visits $(1.0$ percent $[\mathrm{SE}=$ $0.06]$ ) made by patients who required immediate attention, defined as visits where the reason for visit was respiratory arrest, cardiac arrest, cardiopulmonary arrest, or unconsciousness; cardiopulmonary resuscitation or endotracheal intubation was performed; or the patient died in the ED (data not shown).

Arrival and waiting times and duration in the emergency departmentConsistent with previous years, the majority of patients arrived at the ED between 8 a.m. and 10 p.m., although older patients tended to arrive in the late morning and children tended to present in the early evening (15). No differences in arrival time were found for visits triaged as emergent compared with those that were nonurgent, although a higher proportion of older patients was triaged as emergent and arrived in the morning. The similarity of distributions of arrival times for nonurgent and emergent conditions may dispel the belief that less severe cases tend to present to the ED at the convenience of the patient's work schedule.

Waiting time to see the physician in the ED was determined by calculating the difference between time of arrival and time seen by the physician. Visits in which the patient did not see a physician were excluded from the 


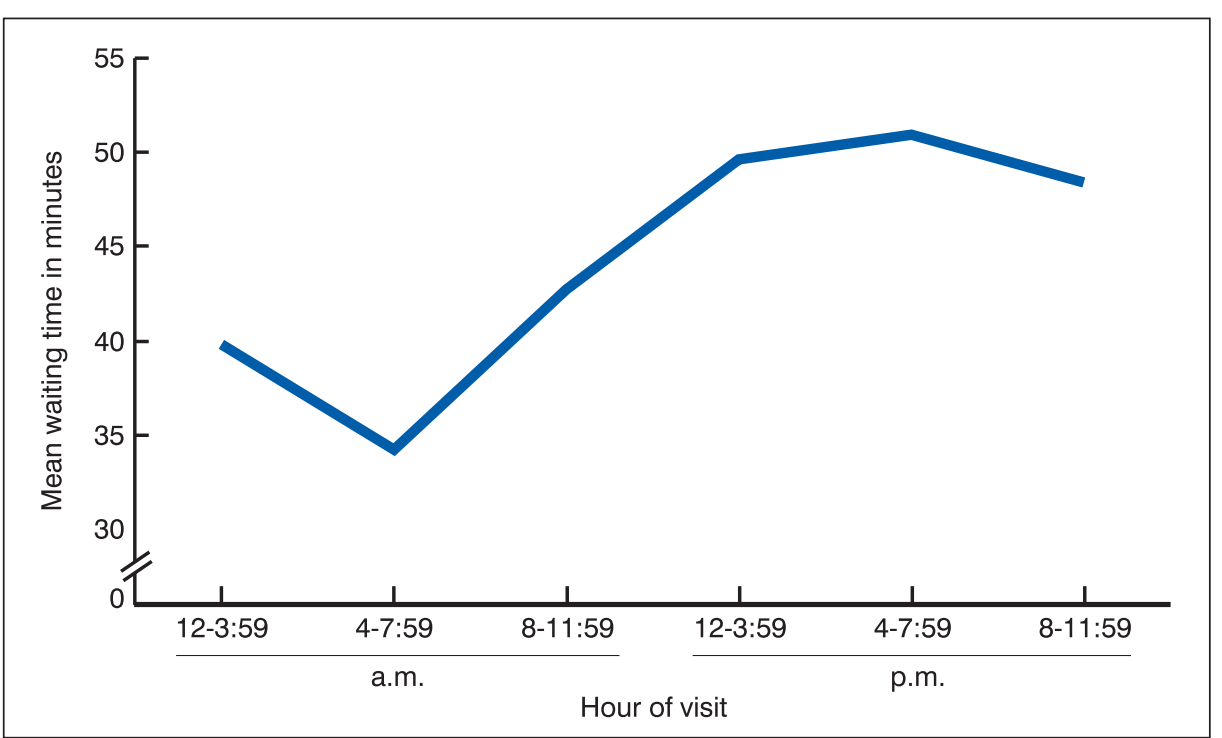

Figure 6. Mean waiting time to see a physician at emergency department visits by hour of visit: United States, 2003

analysis. About three-fifths of patients waited less than 1 hour to see a physician (table 5). On average, patients waited 46.5 minutes before seeing a physician (not significantly different from the observed average when last estimated in 2000 (45.1 minutes) (13). Figure 6 shows that mean waiting time varied by arrival time. Patients arriving between 4 a.m. and 8 a.m. waited the least amount of time, and patients arriving between noon and 8 p.m. waited the longest. Waiting time also varied by expected source of payment.
Visits by Medicare patients had a lower mean waiting time $(39.7, \mathrm{SE}=1.6)$ than those that were to be paid out-of-pocket (53.6, $\mathrm{SE}=4.3$ ) or by Medicaid (49.0, $\mathrm{SE}=2.0$ ) (figure 7). Waiting time was unknown for 17.6 percent of visits. Because Medicare patients are predominantly older and present with more severe conditions, it is expected that waiting times would be shorter compared with other visits by patients with other expected sources of payment. There was no difference between the mean waiting times for patients who

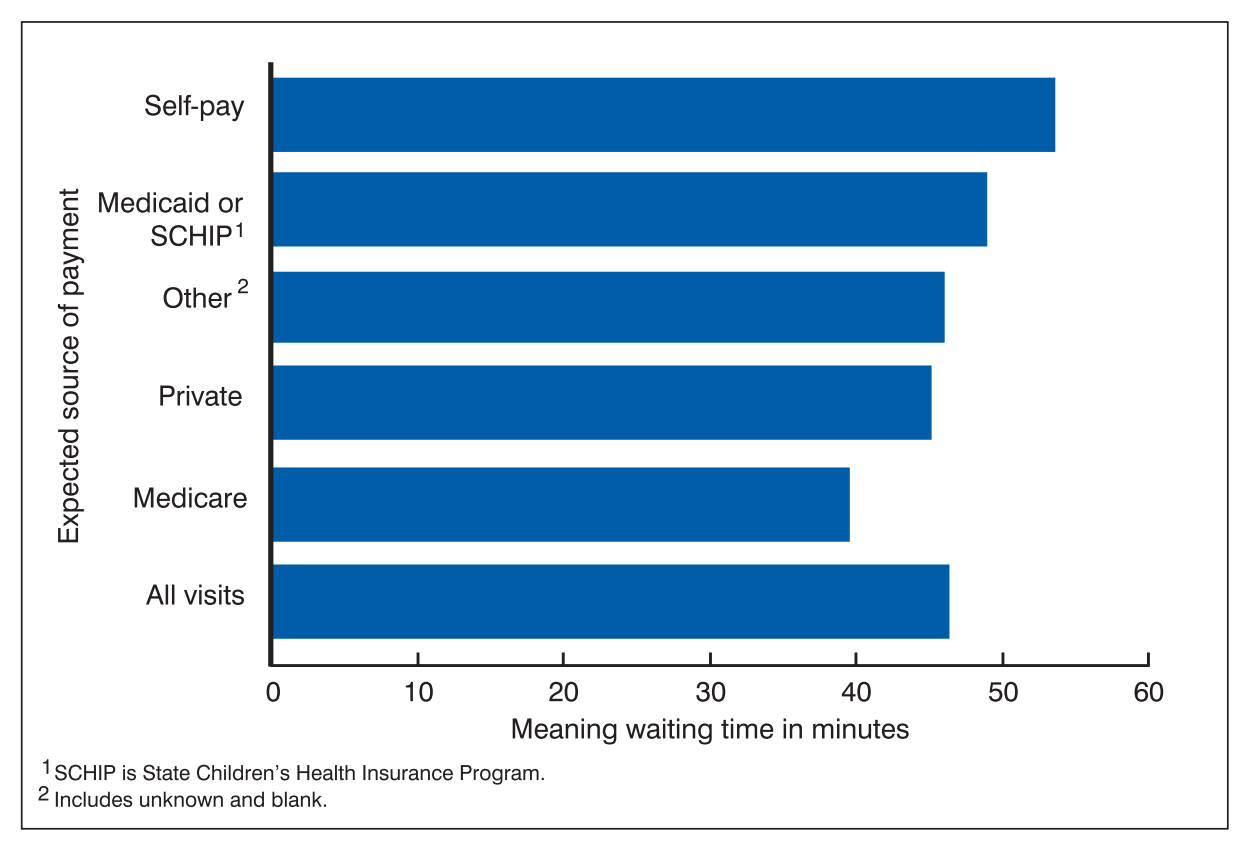

Figure 7. Mean waiting time to see a physician at emergency department visits by expected source of payment: United States, 2003 paid out-of-pocket compared with those who had private insurance $(45.2, \mathrm{SE}=$ 1.7).

Duration in the ED was determined by calculating the difference between time of arrival and time of discharge. The duration for 67.5 percent of ED visits was between 1 and 6 hours (table 5). On average, patients spent 3.2 hours $(\mathrm{SE}=0.1)$ in the emergency department.

Initial vital signs and orientationSince 2001, the NHAMCS has collected the patient's initial vital signs (i.e., temperature, blood pressure, and pulse). The mean temperature for patients complaining of fever was $100.6^{\circ} \mathrm{F}(\mathrm{SE}=$ $0.1)$ or $38.1^{\circ} \mathrm{C}$. At 4.6 percent $(\mathrm{SE}=$ 0.3 ) of illness visits, the patient had a fever of $102^{\circ} \mathrm{F} / 38.9^{\circ} \mathrm{C}$ or higher. When the patient's diagnosis was hypertension, the average measurements for systolic and diastolic blood pressures were 164.1 $(\mathrm{SE}=1.8)$ and $89.5 \mathrm{mmHg}(\mathrm{SE}=0.9)$, respectively. The mean pulse was 92.9 $(\mathrm{SE}=0.4)$ beats per minute (data not shown).

In 2003, a checkbox was added to determine whether the patient was oriented to time, place, and person. The patient was not oriented at 3.7 million visits, or 3.3 percent $(\mathrm{SE}=0.3)$ of all visits (data not shown). The patient was 85 years of age or over at 16.8 percent $(\mathrm{SE}=1.7)$ and was triaged as emergent at 8.8 percent $(\mathrm{SE}=0.8)$ of visits in which the patient was disoriented. Orientation was not indicated for 29.1 percent of all visits.

Patient's principal reason for visit-The principal reason for visit is the main complaint, symptom, or reason the patient came to the ED. Up to three reasons for visit were coded according to A Reason for Visit Classification for Ambulatory Care (RVC) (16). The RVC is a classification scheme developed by NCHS that has been used for over 20 years to code patients' complaints or reasons for seeking care. It is divided into eight modules or groups of reasons as shown in table 6 and includes all the reasons for which patients see their health care provider. This includes symptoms, followup for prior diagnoses, routine examinations and screening, treatment for conditions and operations, various therapies, and injuries. The 
symptom module is further divided into symptoms that refer to specific body systems, such as respiratory or cardiovascular and lymphatic. Each reason is assigned a three- or four-digit classification code (e.g., S260-

"Abnormal pulsations and palpitations," is further detailed to S260.1- "Increased heartbeat," S260.2- "Decreased heartbeat," and S260.3- "Irregular heartbeat").

In 2003, about 73 percent of ED visits were made for reasons classified in the symptom module. Within this module, general symptoms such as fever, fatigue, and pain accounted for 16.2 percent of the total (table 6). Digestive symptoms accounted for 13.3 percent of visits, and musculoskeletal and respiratory symptoms were recorded at 12.9 and 12.0 percent of visits, respectively. The 20 most frequently mentioned principal reasons for visit, representing almost one-half of all visits, are shown in table 7. Stomach and abdominal pain, cramps, and spasms were reported most frequently, accounting for 6.7 percent of all ED visits. Chest pain and fever accounted for 5.1 and 5.0 percent of visits, respectively. Laceration and cuts - upper extremity was the most frequently mentioned reason for visit in the injury module (1.9 percent). It should be noted that estimates differing in ranked order may not be significantly different from each other.

Alcohol-related visits-Although there is a separate item on the Patient Record form to indicate whether the visit was alcohol related, sometimes an alcohol-related reason for visit is specified or an alcohol-related diagnosis is rendered without the alcohol item being checked. Using the checkbox alone provides an estimate of about 2,535,000 visits (2.2 percent, $\mathrm{SE}=0.1$ ) related to the use of alcohol by the patient or another person or both (data not shown). The alcohol checkbox was marked "unknown" or left blank for 11.8 percent of visits. Using data from the checkbox, any alcohol-related reasons for visit, any alcohol-related external causes of injury, and any alcohol-related diagnoses produced an estimate of 4,003,000 visits (3.5 percent, $\mathrm{SE}=0.2$ ). This approach resulted in a better indication that the visit involved alcohol than using the reason-for-visit module (16), external cause of injury code developed by NCHS, International Classification of Diseases, 9th Revision Clinical Modification (ICD-9-CM) (17) diagnosis, or the unedited alcohol item alone. All of the estimates presented below are based on both the alcohol checkbox and the alcohol-related reason for visit, external cause of injury, and diagnosis items. The percent distribution of alcohol-related ED visits by age is as follows: 11.4 percent $(\mathrm{SE}=1.1$ ) for adolescents and young adults under 21 years of age; 53.2 percent $(\mathrm{SE}=1.8)$ for persons 21-44 years of age; 27.9 percent $(\mathrm{SE}=1.9)$ for middle-aged persons 45-64 years of age; and 7.5 percent (SE $=1.1$ ) for seniors 65 years of age and over. Among persons under 21 years of age, 1.3 percent $(\mathrm{SE}=0.1)$ of visits were alcohol related compared with 5.2 percent $(\mathrm{SE}=0.3)$ and 5.3 percent $(\mathrm{SE}=0.4)$ of visits made by persons 21-44 years of age and middle-aged adults 45-64 years of age, respectively. Of all alcohol-related visits, 66.7 percent ( $\mathrm{SE}=1.7)$ were injury related.

Work-related visits-In 2003, the definition of a work-related ED visit was expanded to include illnesses as well as injuries that happened while the patient was engaged in work activities occurring on or off the employer's premises. In previous reports, estimates of work-related ED visits have been presented for injury visits only. During 2003, there were 3,134,000 work-related $\mathrm{ED}$ visits, and 94.0 percent $(\mathrm{SE}=1.0)$ of these were related to injury (data not shown). Persons 18-64 years of age made 96.2 percent $(\mathrm{SE}=0.7)$ of the work-related visits, and 4.9 percent (SE $=0.2$ ) of their ED visits were work related. The work checkbox was marked "unknown" or left blank for 6.7 percent of all visits.

Prior ED visit-The survey collected two items designed to measure prior use of the ED-whether the patient had been seen in the ED within the past 72 hours and the episode of care (i.e., whether the sampled visit was for followup versus an initial visit for a problem). At 2.8 percent of visits, the patient had been seen in the ED within the last 72 hours (table 8). This percentage did not vary by age, race, sex, type of payment, injury or illness, or disposition. For about 89 percent of visits, this ED visit was the initial visit for the patient's problem, and for 5.5 percent it was a followup visit.

Primary diagnosis-Hospital staff was asked to record the primary diagnosis or problem associated with the patient's most important reason for the current visit and any other significant current diagnoses. Up to three diagnoses were coded according to the ICD-9-CM (17). Displayed in table 9 are ED visits by primary diagnosis, using the major disease categories specified by the ICD-9-CM. Injury and poisoning diagnoses accounted for 25.6 percent of all visits. Symptoms, signs, and ill-defined conditions and diseases of the respiratory system accounted for 18.7 percent and 12.3 percent, respectively. The most frequently reported primary diagnoses for 2003 are shown in table 10. Contusion leads the list (4.2 percent), followed by acute upper respiratory infection, excluding pharyngitis (4.0 percent); abdominal pain (3.9 percent); chest pain (3.7 percent); and open wound, excluding head (3.6 percent). The leading diagnosis by age group was as follows: infants and children (under 13 years of age) - acute respiratory infection, excluding pharyngitis; adolescents and young adults (13-21 years of age)—contusions; adults (22-49 years of age)_abdominal pain; middle-aged persons (50-64 years of age)_chest pain; and seniors (65 years of age and over)—-heart disease, excluding ischemic (table 11).

Injury-or poisoning-related visits - EDs are the primary health care setting where acute medical problems and severe injuries are treated; therefore, it is helpful to understand the relative burden of illness versus injury cases on overall utilization. Although there is a separate item on the Patient Record form to indicate whether the visit was for an injury or poisoning or adverse effect of medical treatment, sometimes an injury reason for visit is specified or an injury diagnosis is rendered without the injury item being checked. Therefore, the visit is counted as an injury visit and the checkbox is coded to 
"yes" if any of the three reasons for visit were in the injury module or any of the three diagnoses were in the injury or poisoning chapter of the ICD-9-CM (17). This provides a better indicator that the visit involves an injury than using the reason-for-visit module,

ICD-9-CM injury diagnosis, or unedited injury item alone.

Approximately 40.2 million ED visits were made for injury or poisoning, which represented 35.3 percent of all ED visits. There were 14.1 injury- or poisoning-related visits per 100 persons (table 12). About 70 percent of all injury visits occurred among persons younger than 45 years of age. Persons 15-24 years of age had the highest injuryrelated visit rate. Males had a higher injury-related visit rate than females overall and for the age groups under 15 years and 25-44 years. The injuryrelated visit rate for black or African American persons was higher than for white persons, with the overall rate for the former being driven by visits among persons 15-64 years of age. Rates were higher in the Northeast than in the West.

About 6.6 percent $(\mathrm{SE}=0.3)$ of all injury-related visits were alcohol related. Alcohol-related visits were assessed using the alcohol checkbox and alcohol-related reason for visit, cause of injury, and diagnosis items on the Patient Record form. For all injury visits, 7.3 percent $(\mathrm{SE}=0.4)$ were work related.

Intentionality of injury was collected using a checkbox item that indicated that 75.2 percent $(\mathrm{SE}=0.8)$ of injuries were unintentional; 7.9 percent $(\mathrm{SE}=0.4)$ were intentional; and for 17.0 percent $(\mathrm{SE}=0.8)$ of visits, intent was unknown (data not shown). Of the intentional injuries, 52.9 percent were the result of an assault, and 47.1 percent were self-inflicted.

Table 13 shows ED visits by the intent and mechanism of the first-listed external cause-of-injury codes (E-codes). Up to three external causes of injury were coded according to the Supplementary Classification of External Causes of Injury and Poisoning in the ICD-9-CM (17). External cause was not provided for 15.7 percent of injury visits. About 70 percent of injury-related visits were the result of an unintentional injury. The reader should keep in mind that the results regarding intentionality of the injury in table 13 will vary from those obtained by using the checkbox. Intentionality of the injury, as described in the previous paragraph, is based on responses to the checkbox item on the Patient Record form, rather than on the ICD-9-CM groupings used in table 13. Discrepancies may arise in respondent interpretation of intent. For example, in some cases, hospital staff checked the "assault" category for dog bite injuries. However, dog bites are an unintentional injury based on the ICD-9-CM E-codes. Also, the exact cause of injury is missing more frequently than the checkbox entry.

Unintentional injuries due to falls (19.9 percent), striking against or being struck accidentally by objects or persons (10.5 percent), and motor vehicle traffic-related injuries (10.4 percent) accounted for the largest proportion of injury-related ED visits. Four percent of injury-related ED visits were the result of assaults. An unarmed fight or brawl was the leading reason for assaultrelated injuries (2.6 percent). Selfinflicted injuries resulted in 438,000 ED visits (1.1 percent), with poisoning being the most frequent cause (0.8 percent). Approximately 1.7 million ED visits were for adverse effects of medical treatment and represented 4.2 percent of injury- or poisoning-related ED visits. This included adverse drug reactions and complications from surgical and medical procedures.

The Barell Injury Diagnosis Matrix: Classification of Region of Body and Nature of the Injury was used in table 14 to show the distribution of injury-related visits by body site of primary diagnosis (18). A detailed description of the ICD-9-CM codes used to create the body site of primary diagnosis reclassification coding is provided in the "Technical Notes." The most commonly mentioned body site was hand, wrist, and fingers (11.7 percent), followed by lower leg and ankle (4.7 percent) and face (4.6 percent).

Diagnostic and screening services-Diagnostic and screening services were ordered or provided by hospital staff at 89.7 percent of ED visits, up from 86.8 percent in 2002 (table 15). Frequently mentioned services included medical screening exam (68.5 percent), complete blood count (CBC) (33.0 percent), pulse oximetry (23.4 percent), chest $\mathrm{x}$ ray (19.3 percent), other blood tests (19.2 percent), and urinalysis (17.7 percent). Imaging was provided at 42.9 percent of visits. Blood tests and cultures were ordered at 36.2 percent and 9.7 percent of visits, respectively. Note that for items related to diagnostic and screening services, procedures, providers seen, and disposition, hospital staff was asked to check all of the applicable categories for each item. Therefore, multiple responses could be coded for each visit. About 9 percent of ED visits had no diagnostic or screening services. Figure 8 shows that the rate of diagnostic and screening services ordered or provided at ED visits increased with age.

Procedures-Procedures were provided at 45.4 percent of ED visits. For visits with procedures, 83.8 percent had only one procedure recorded. The most frequently mentioned procedures were the administration of intravenous fluids (21.8 percent), wound care (9.8 percent), and orthopedic care (5.4 percent) (table 16). Figure 8 shows that the rate of procedures provided at ED visits increased with age. Mentions of procedures were missing for 6.7 percent of visits.

Medication therapy-Hospital staff was instructed to record all new or continued medications ordered, supplied, or administered at the visit. This included prescription and nonprescription preparations, immunizations, desensitizing agents, and anesthetics. Starting in 2003, the NHAMCS collected up to eight medications (as opposed to six). In this survey, recorded medications are referred to as drug mentions and are coded according to a classification system developed at NCHS. A report describing the method and instruments used to collect and process drug information is available (19). As used in the NHAMCS, the term "drug" is interchangeable with the term "medication." The term "prescribing" is used broadly to mean ordering or providing any medication, whether 

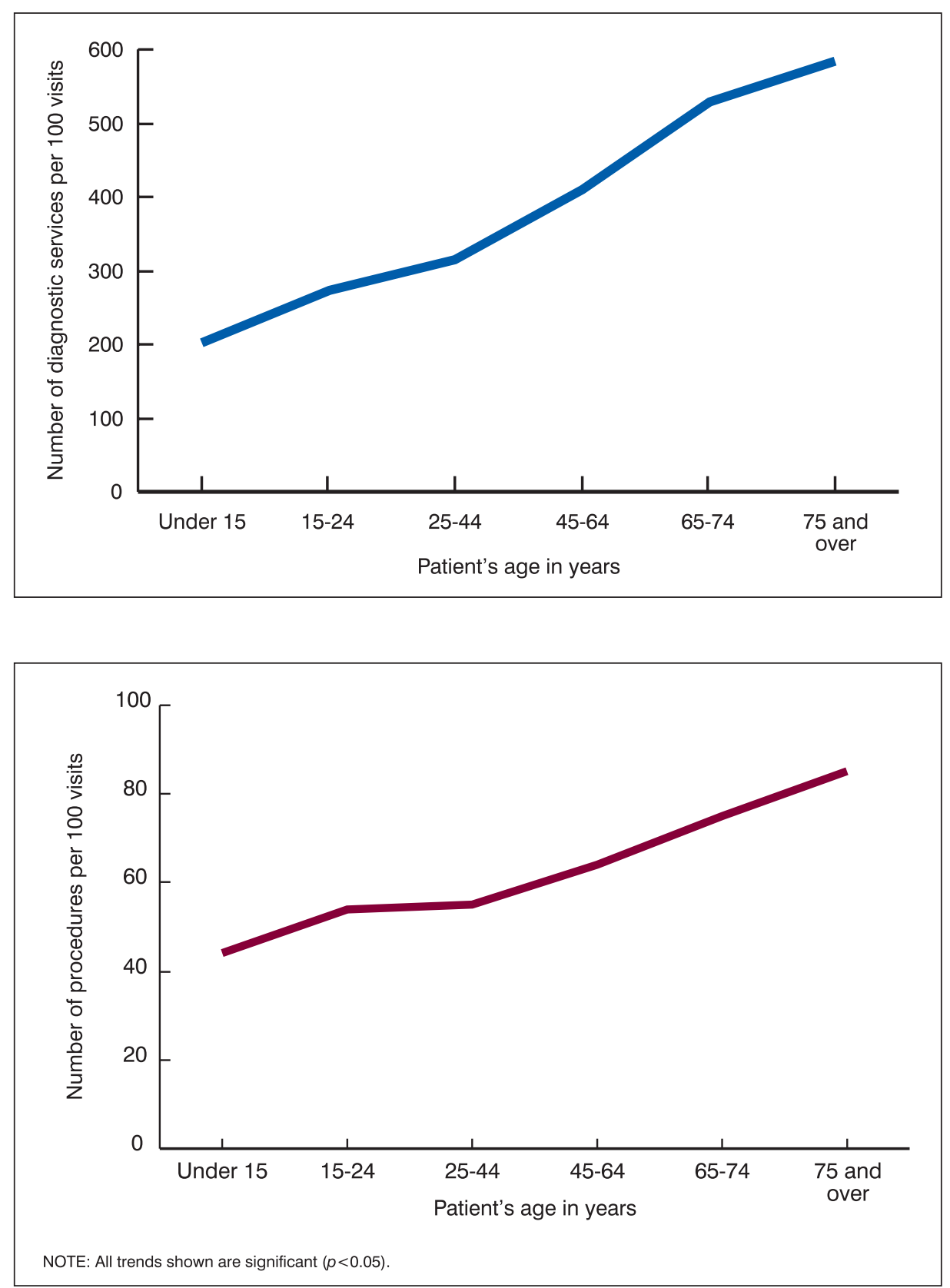

Figure 8. Diagnostic service and procedure rates at emergency department visits by age: United States, 2003

prescription or over-the-counter. Visits with one or more drug mentions are termed "drug visits" in the NHAMCS.

Medications were used at 77.3 percent $(\mathrm{SE}=0.8)$ of all $\mathrm{ED}$ visits, up by 4 percent since 1993 (table 17). There was an average of 1.9 drug mentions per ED visit. For visits where medications were mentioned, there was an average of 2.4 drugs provided per visit. About 2 percent of visits had as many as eight or more drugs prescribed. There were 212.2 million drug mentions at ED visits during 2003 (table 18 footnote).
Drug mentions are shown by therapeutic subclasses in table 18. This classification is based on the four-digit therapeutic categories used in the National Drug Code Directory, 1995 edition (20). Drugs may have more than one therapeutic application, and in the NHAMCS, up to three therapeutic drug classes are recorded for each drug. Prior to 2002, a drug was classified under its primary therapeutic use and data were presented for two-digit therapeutic classification codes. Beginning in 2002, drug data are being shown for up to three therapeutic subclassifications at the four-digit level. The leading drug subclasses in 2003 were narcotic analgesics (14.1 percent) and nonsteroidal anti-inflammatory agents (12.1 percent). The 20 most frequently used generic substances for 2003 are shown in table 19. Drug products containing more than one ingredient (combination products) are included in the data for each ingredient. For example, acetaminophen with codeine is included in both the count for acetaminophen and the count for codeine. The most frequently occurring generic substances in drugs mentioned at ED visits were acetaminophen, ibuprofen, promethazine, and ketorolac tromethamine.

The 20 most frequently mentioned medications are shown in table 20 according to the name written on the ED Patient Record form by hospital staff. This could be a brand name, generic name, or therapeutic effect. Tylenol, which is classified as a nonnarcotic analgesic and antipyretic, was the drug most frequently mentioned, accounting for 5.2 percent of all ED drug mentions. Motrin, which is classified as an NSAID and antiarthritic, was listed for 4.6 percent of ED drug mentions. Other frequent drug mentions were Phenergan (4.0 percent), Vicodin (3.3 percent), and Toradol (2.9 percent).

Providers seen-Staff was asked to check all of the providers seen during the visit. Multiple responses could be coded per visit. A physician was seen at 92.4 percent of visits, and a registered nurse attended the patient at 90.5 percent of ED visits (table 21). A resident and/or intern was seen at 8.4 percent of visits. For 8.7 percent of visits, a physician other than a staff physician or a resident and/or intern was seen. At 7.6 percent of visits, the patient was not attended by a physician. The provider item was not checked for 0.9 percent of visits.

Visit disposition-Staff was asked to record visit disposition and instructed that multiple responses could be coded for this item. Forty-seven percent of ED visits resulted in a referral to another physician or clinic for followup (table 22). For 41.3 percent of visits, patients were told to return to the ED if 
needed or by appointment. Patients were told to return to the referring physician at 13.9 percent of visits. About 14 percent of ED visits resulted in hospital admission. This included direct admission to the intensive care unit, critical care unit, or coronary care unit, which occurred in about 1 out of 10 admissions. Patients were admitted to a 23-hour observation unit at less than 1 percent of visits. For 6.3 percent of visits, no followup was planned. An increasing trend in this visit disposition was previously reported from 1992 through 1999 (9), and it continued through 2001 (up by 60.0 percent), when it peaked. For the first time, in 2003 the proportion of visits with this disposition decreased to the 1992 level. The patient left before being seen by a physician and against medical advice at 1.7 and 1.0 percent of visits, respectively. At less than 1 percent of visits, the patient was referred to an alcohol or drug treatment program or advised to return to nonphysician treatment or a support service.

\section{Hospital ED-level estimates}

In 2003, about 55 percent $(\mathrm{SE}=$ 2.3) of all hospital EDs had annual visit volumes less than 20,000 visits, and 11.7 percent $(\mathrm{SE}=1.3)$ had volumes greater than 50,000 visits (data not shown). Hospital ED-level estimates for 24-hour EDs are shown in table 23 by size of ED, as measured by annual visit volume (small, medium, and large). Approximately 58 percent of all hospital EDs were located in MSAs (table 23), but they represented 81.5 percent of the annual ED encounters (table 1).

Although EDs in MSAs tended to have a larger volume of visits than in non-MSAs, the ED utilization rate per 100 persons did not vary by the MSA status of the hospital (table 1). About 31.5 percent of EDs, compared with 42.4 percent of visits, were in hospitals affiliated with medical schools. Approximately 47 percent of hospitals with 24-hour EDs had a three-level nursing triage system, and 19.6 percent had four or five levels each. Boardcertified emergency medicine (EM) specialists were available 24 hours a day and 7 days a week in the majority of
EDs (63.5 percent), and pediatric EM specialists were only found in 18.1 percent of EDs. About 45 percent of all hospitals with 24-hour EDs also had OPD clinics offering physician services as opposed to OPDs that only offered ancillary services such as radiology, laboratory, and so on.

Forthcoming reports highlight findings from the special supplement surveys conducted during 2003 on topics ranging from ambulance diversions to preparedness of the hospitals to treat bioterrorism and mass casualty incidents and to treat pediatric emergencies. Additional information about ED utilization is available from the NCHS Ambulatory Health Care Web site: www.cdc.gov/nchs/about/major/ ahcd/ahcd1.htm.

Individual-year reports and public-use data files are available for download from the Web site. Data from the 2003 NHAMCS will also be available on CD-ROM. These and other products can be obtained by contacting the NCHS Ambulatory Care Statistics Branch at (301) 458-4600. Queries regarding NHAMCS data may be sent to NCHS at nchsquery@cdc.gov.

\section{References}

1. Burt CW, Schappert SM. Ambulatory care visits to physician offices, hospital outpatient departments, and emergency departments: United States: 1999-2000. National Center for Health Statistics. Vital Health Stat 13(157). 2004.

2. Asplin BR, Magid DJ, Rhodes KV, et al. A conceptual model of emergency department crowding. Ann Emerg Med. 42:173-80. 2003.

3. Yamane K. Hospital emergency departments-crowded conditions vary among hospitals and communities. GAO-03-460. Washington. U.S. General Accounting Office. 2003.

4. National Center for Health Statistics. Health, United States, 2005 With Chartbook on Trends in the Health of Americans. Hyattsville, MD. 2005. In preparation.

5. Fuda KK. Recent analysis of Massachusetts emergency department data. Presented at the National Association of Health Data
Organizations Annual Meeting. Washington. 2004.

6. Zuckerman S, Shen Y. Characteristics of occasional and frequent emergency department users. Medical Care. 42:176-82. 2004.

7. Middleton KR, Hing ES. National Hospital Ambulatory Medical Care Survey: 2003 outpatient department summary. Advance data from vital and health statistics. Hyattsville, MD: National Center for Health Statistics. 2005. In preparation.

8. Hing ES, Cherry D, Woodwell DA. National Ambulatory Medical Care Survey: 2003 summary. Advance data from vital and health statistics. Hyattsville, MD: National Center for Health Statistics. 2005. In preparation.

9. Burt CW, McCaig LF. Trends in hospital emergency department utilization: United States, 1992-99. National Center for Health Statistics. Vital Health Stat 13(150). 2001.

10. McCaig LF, McLemore T. Plan and operation of the National Hospital Ambulatory Medical Care Survey. National Center for Health Statistics. Vital Health Stat 1(34). 1994.

11. Research Triangle Institute (2004). SUDAAN (Release 9.0) [Computer Software]. Research Triangle Park, NC: Research Triangle Institute.

12. American Hospital Association (personal communication-Scott Bates). 2003.

13. McCaig LF, Ly N. National Hospital Ambulatory Medical Care Survey: 2000 emergency department summary. Advance data from vital and health statistics; no 326. Hyattsville, MD: National Center for Health Statistics. 2002.

14. Schafermeyer RW, Asplin BR. Hospital and emergency department overcrowding in the United States. Emerg Med. 15:22-27. 2003.

15. McCaig LF, Burt CW. National Hospital Ambulatory Medical Care Survey: 2001 emergency department summary. Advance data from vital and health statistics; no 335.

Hyattsville, Maryland: National Center for Health Statistics. 2003.

16. Schneider D, Appleton L, McLemore T. A reason for visit classification for ambulatory care. National Center for Health Statistics. Vital and Health Stat 2(78). 1979.

17. Public Health Service and Health Care Financing Administration. International Classification of 
Diseases, 9th Revision, Clinical

Modification, 6th ed., Washington. Public Health Service. 1998.

18. www.cdc.gov/nchs/about/otheract/ice/ barellmatrix.htm.

19. Koch H, Campbell W. The collection and processing of drug information: National Ambulatory Medical Care Survey, United States, 1980. National Center for Health Statistics. Vital Health Stat 2(90). 1982.

20. Food and Drug Administration. National Drug Code Directory, 1995 edition. Washington. Public Health Service. 1995.

21. National Center for Health Statistics. Public-Use Data File Documentation. 2003 National Hospital Ambulatory Medical Care Survey. Hyattsville, MD. 2005. In preparation.

22. Office of Management and Budget (1997), Statistical Policy Directive no. 15, Race and Ethnic Standards for Federal Statistics and Administrative Reporting, Federal Register; Part II:36873-946, July 7, 1997. 
Table 1. Number, percent distribution, and annual rate of emergency department visits with corresponding standard errors, by selected patient and hospital characteristics: United States, 2003

\begin{tabular}{|c|c|c|c|c|c|c|}
\hline Selected patient and hospital characteristics & $\begin{array}{l}\text { Number of } \\
\text { visits in } \\
\text { thousands }\end{array}$ & $\begin{array}{l}\text { Standard } \\
\text { error in } \\
\text { thousands }\end{array}$ & $\begin{array}{c}\text { Percent } \\
\text { distribution }\end{array}$ & $\begin{array}{c}\text { Standard } \\
\text { error of } \\
\text { percent }\end{array}$ & $\begin{array}{l}\text { Number of } \\
\text { visits per } \\
100 \text { persons } \\
\text { per year }^{1,2}\end{array}$ & $\begin{array}{l}\text { Standard } \\
\text { error of } \\
\text { rate }\end{array}$ \\
\hline All visits $\ldots \ldots \ldots \ldots \ldots \ldots \ldots$ & 113,903 & 4,094 & 100.0 & $\ldots$ & 39.9 & 1.4 \\
\hline \multicolumn{7}{|l|}{ Patient characteristics } \\
\hline \multicolumn{7}{|l|}{ Age: } \\
\hline Under 15 years $\ldots \ldots \ldots \ldots \ldots \ldots \ldots$ & 24,733 & 1,521 & 21.7 & 0.9 & 40.8 & 2.5 \\
\hline 15-24 years . . . . . . . . . . . . . . . & 17,731 & 703 & 15.6 & 0.3 & 44.2 & 1.8 \\
\hline $25-44$ years $\ldots \ldots \ldots \ldots \ldots \ldots$ & 32,906 & 1,274 & 28.9 & 0.5 & 40.0 & 1.5 \\
\hline $45-64$ years $\ldots \ldots \ldots \ldots \ldots \ldots$ & 20,992 & 824 & 18.4 & 0.4 & 30.8 & 1.2 \\
\hline $65-74$ years $\ldots \ldots \ldots \ldots \ldots \ldots$ & 7,153 & 344 & 6.3 & 0.2 & 39.5 & 1.9 \\
\hline 75 years and over $\ldots \ldots \ldots \ldots \ldots$ & 10,389 & 476 & 9.1 & 0.3 & 64.2 & 2.9 \\
\hline \multicolumn{7}{|l|}{ Sex and age: } \\
\hline 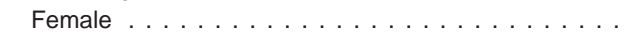 & 60,995 & 2,212 & 53.5 & 0.4 & 41.8 & 1.5 \\
\hline Under 15 years . . . . . . . . . . . . . . & 11,209 & 713 & 9.8 & 0.5 & 37.8 & 2.4 \\
\hline $15-24$ years $\ldots \ldots \ldots \ldots \ldots \ldots \ldots$ & 10,558 & 447 & 9.3 & 0.2 & 53.1 & 2.2 \\
\hline $25-44$ years $\ldots \ldots \ldots \ldots \ldots \ldots$ & 17,976 & 750 & 15.8 & 0.4 & 43.2 & 1.8 \\
\hline $45-64$ years . . . . . . . . . . . . . . . & 11,220 & 466 & 9.9 & 0.2 & 31.9 & 1.3 \\
\hline $65-74$ years $\ldots \ldots \ldots \ldots \ldots \ldots$ & 3,811 & 211 & 3.3 & 0.1 & 38.7 & 2.1 \\
\hline 75 years and over $\ldots \ldots \ldots \ldots \ldots$ & 6,220 & 285 & 5.5 & 0.2 & 62.7 & 2.9 \\
\hline 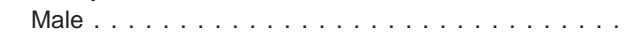 & 52,908 & 1,961 & 46.5 & 0.4 & 37.9 & 1.4 \\
\hline Under 15 years . . . . . . . . . . . . . . & 13,524 & 845 & 11.9 & 0.5 & 43.6 & 2.7 \\
\hline 15-24 years . . . . . . . . . . . . . . & 7,172 & 294 & 6.3 & 0.2 & 35.4 & 1.5 \\
\hline 25-44 years . . . . . . . . . . . . . . & 14,929 & 625 & 13.1 & 0.3 & 36.8 & 1.5 \\
\hline $45-64$ years . . . . . . . . . . . . . . & 9,772 & 399 & 8.6 & 0.2 & 29.5 & 1.2 \\
\hline $65-74$ years . . . . . . . . . . . . . . . & 3,342 & 177 & 2.9 & 0.1 & 40.6 & 2.2 \\
\hline 75 years and over $\ldots \ldots \ldots \ldots \ldots$ & 4,169 & 235 & 3.7 & 0.2 & 66.5 & 3.7 \\
\hline \multicolumn{7}{|l|}{ Race and age: ${ }^{3}$} \\
\hline 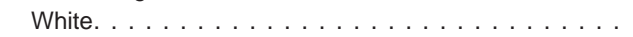 & 85,833 & 3,642 & 75.4 & 1.3 & 37.2 & 1.6 \\
\hline Under 15 years . . . . . . . . . . . . . . . & 17,977 & 1,226 & 15.8 & 0.8 & 38.8 & 2.6 \\
\hline 15-24 years . . . . . . . . . . . . . . & 12,852 & 608 & 11.3 & 0.3 & 41.1 & 1.9 \\
\hline $25-44$ years . . . . . . . . . . . . . . . & 24,312 & 1,087 & 21.3 & 0.5 & 37.0 & 1.7 \\
\hline $45-64$ years $\ldots \ldots \ldots \ldots \ldots \ldots$ & 16,050 & 730 & 14.1 & 0.4 & 28.1 & 1.3 \\
\hline $65-74$ years . . . . . . . . . . . . . . . & 5,692 & 292 & 5.0 & 0.2 & 36.4 & 1.9 \\
\hline 75 years and over $\ldots \ldots \ldots \ldots \ldots$ & 8,950 & 446 & 7.9 & 0.3 & 62.0 & 3.1 \\
\hline Black or African American . . . . . . . . . . . . . & 24,740 & 1,578 & 21.7 & 1.3 & 69.3 & 4.4 \\
\hline Under 15 years . . . . . . . . . . . . . . & 5,855 & 600 & 5.1 & 0.5 & 62.0 & 6.3 \\
\hline $15-24$ years $\ldots \ldots \ldots \ldots \ldots \ldots \ldots$ & 4,385 & 353 & 3.9 & 0.3 & 75.7 & 6.1 \\
\hline $25-44$ years $\ldots \ldots \ldots \ldots \ldots \ldots \ldots$ & 7,576 & 487 & 6.7 & 0.4 & 73.4 & 4.7 \\
\hline $45-64$ years . . . . . . . . . . . . . . & 4,425 & 319 & 3.9 & 0.3 & 60.5 & 4.4 \\
\hline $65-74$ years . . . . . . . . . . . . . . . & 1,265 & 143 & 1.1 & 0.1 & 76.7 & 8.7 \\
\hline 75 years and over $\ldots \ldots \ldots \ldots$ & 1,232 & 121 & 1.1 & 0.1 & 102.9 & 10.1 \\
\hline 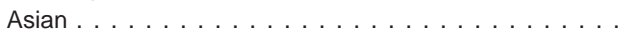 & 2,030 & 246 & 1.8 & 0.2 & 17.1 & 2.1 \\
\hline Native Hawaiian or other Pacific Islander . . . . . . . . . & *457 & 146 & ${ }^{*} 0.4$ & 0.1 & ${ }^{*} 94.0$ & 30.1 \\
\hline American Indian or Alaska Native. . . . . . . . . . . . . . & 682 & 171 & 0.6 & 0.1 & 24.9 & 6.2 \\
\hline Multiple races $\ldots \ldots \ldots \ldots \ldots \ldots \ldots$ & 162 & 41 & 0.1 & 0.0 & 3.8 & 1.0 \\
\hline
\end{tabular}

See footnotes at end of table. 
Table 1. Number, percent distribution, and annual rate of emergency department visits with corresponding standard errors, by selected patient and hospital characteristics: United States, 2003-Con.

\begin{tabular}{|c|c|c|c|c|c|c|}
\hline Selected patient and hospital characteristics & $\begin{array}{l}\text { Number of } \\
\text { visits in } \\
\text { thousands }\end{array}$ & $\begin{array}{l}\text { Standard } \\
\text { error in } \\
\text { thousands }\end{array}$ & $\begin{array}{c}\text { Percent } \\
\text { distribution }\end{array}$ & $\begin{array}{c}\text { Standard } \\
\text { error of } \\
\text { percent }\end{array}$ & $\begin{array}{c}\text { Number of } \\
\text { visits per } \\
100 \text { persons } \\
\text { per year }^{1,2}\end{array}$ & $\begin{array}{l}\text { Standard } \\
\text { error of } \\
\text { rate }\end{array}$ \\
\hline \multicolumn{7}{|l|}{ Hospital characteristics } \\
\hline \multicolumn{7}{|l|}{ Ownership: } \\
\hline 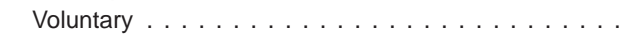 & 82,170 & 4,419 & 72.1 & 2.5 & 28.8 & 1.5 \\
\hline Government . . . . . . . . . . . . . . . & 21,116 & 2,541 & 18.5 & 2.2 & 7.4 & 0.9 \\
\hline Proprietary . . . . . . . . . . . . . . . & 10,617 & 1,699 & 9.3 & 1.5 & 3.7 & 0.6 \\
\hline \multicolumn{7}{|l|}{ Geographic region: } \\
\hline Northeast . . . . . . . . . . . . . . . . . & 23,814 & 1,648 & 20.9 & 1.4 & 44.4 & 3.1 \\
\hline Midwest . . . . . . . . . . . . . . . . & 25,205 & 1,697 & 22.1 & 1.4 & 39.2 & 2.6 \\
\hline 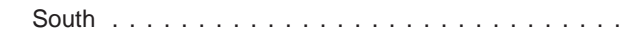 & 44,958 & 2,717 & 39.5 & 1.8 & 44.0 & 2.7 \\
\hline 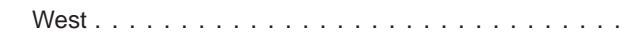 & 19,926 & 2,115 & 17.5 & 1.6 & 30.5 & 3.2 \\
\hline \multicolumn{7}{|l|}{ Metropolitan status: } \\
\hline $\mathrm{MSA}^{4} \ldots \ldots \ldots \ldots \ldots \ldots$ & 92,847 & 4,106 & 81.5 & 2.0 & 38.9 & 1.7 \\
\hline Non-MSA ${ }^{4} \ldots \ldots \ldots \ldots$ & 21,056 & 2,370 & 18.5 & 2.0 & 44.7 & 5.0 \\
\hline
\end{tabular}

... Category not applicable.

* Figure does not meet standard of reliability or precision.

0.0 Quantity more than zero but less than 0.05 .

${ }^{1}$ Visit rates for age, sex, race, and region are based on the July 1, 2003, set of estmates of the civilian noninstitutional population of the United States as developed by the Population Division, U.S. Census Bureau. These population estimates reflect Census 2000 data and are available from the Census Bureau. See "Technical Notes" for more details.

${ }^{2}$ Population estimates of metropolitan statistical area status are based on data from the 2003 National Health Interview Survey, National Center for Health Statistics, adjusted to the U.S. Census Bureau definition of core-based statistical areas as of December 2003. See www.census.gov/population/www/estimates/metrodef.html for more about metropolitan statistical area definitions. ${ }^{3}$ The race groups, white, black or African American, Asian, Native Hawaiian or other Pacific Islander, American Indian or Alaska Native, and multiple races, include persons of Hispanic and not Hispanic origin. Persons of Hispanic origin may be of any race. Starting with data year 1999, race-specific estimates have been tabulated according to 1997 Standards for Federal Data on Race and Ethnicity and are not strictly comparable with estimates for earlier years. However, the percent of visit records with multiple races indicated is small and lower than what is typically found for self-reported race. See "Technical Notes" for more details.

${ }^{4} \mathrm{MSA}$ is metropolitan statistical area.

NOTE: Numbers may not add to totals because of rounding. 
Table 2. Percent distribution of emergency department visits with corresponding standard errors by patient's mode of arrival, according to patient's age: United States, 2003

\begin{tabular}{|c|c|c|c|c|c|c|}
\hline \multirow[b]{2}{*}{ Patient's age } & \multicolumn{6}{|c|}{ Patient's mode of arrival } \\
\hline & $\begin{array}{l}\text { Number of } \\
\text { visits in } \\
\text { thousands }\end{array}$ & Total & Walk-in & Ambulance & $\begin{array}{l}\text { Public } \\
\text { service }\end{array}$ & $\begin{array}{c}\text { Unknown } \\
\text { or blank }\end{array}$ \\
\hline & \multicolumn{6}{|c|}{ Percent distribution } \\
\hline 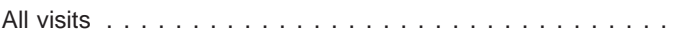 & 113,903 & 100.0 & 79.1 & 14.2 & 1.3 & 5.4 \\
\hline \multicolumn{7}{|l|}{ Age } \\
\hline Under 15 years $\ldots \ldots \ldots \ldots \ldots \ldots \ldots \ldots$ & 24,733 & 100.0 & 88.2 & 3.8 & ${ }^{\star} 0.8$ & 7.2 \\
\hline 15-24 years. . . . . . . . . . . . . . . . . . . & 17,731 & 100.0 & 83.8 & 9.5 & 1.7 & 5.1 \\
\hline 25-44 years . . . . . . . . . . . . . . . . . & 32,906 & 100.0 & 82.6 & 11.3 & 1.4 & 4.7 \\
\hline $45-64$ years . . . . . . . . . . . . . . . . & 20,992 & 100.0 & 76.5 & 17.3 & 1.3 & 4.9 \\
\hline $65-74$ years . . . . . . . . . . . . . . . . . & 7,153 & 100.0 & 66.3 & 27.5 & 1.3 & 4.9 \\
\hline 75 years and over $\ldots \ldots \ldots \ldots \ldots \ldots$ & 10,389 & 100.0 & 52.8 & 40.9 & *1.7 & 4.7 \\
\hline & \multicolumn{6}{|c|}{ Standard error of percent } \\
\hline All visits $\ldots \ldots \ldots \ldots \ldots \ldots \ldots \ldots$ & $\ldots$ & $\ldots$ & 0.9 & 0.5 & 0.2 & 0.8 \\
\hline \multicolumn{7}{|l|}{ Age } \\
\hline Under 15 years . . . . . . . . . . . . . . . . . . . & $\ldots$ & $\ldots$ & 1.8 & 0.3 & 0.2 & 1.7 \\
\hline 15-24 years. . . . . . . . . . . . . . . . . . & $\ldots$ & $\ldots$ & 1.1 & 0.6 & 0.2 & 0.9 \\
\hline 25-44 years . . . . . . . . . . . . . . . . . . & $\ldots$ & $\ldots$ & 0.9 & 0.5 & 0.2 & 0.7 \\
\hline 45-64 years . . . . . . . . . . . . . . . . . & $\ldots$ & $\ldots$ & 0.9 & 0.7 & 0.3 & 0.7 \\
\hline $65-74$ years . . . . . . . . . . . . . . . . . . & $\ldots$ & $\ldots$ & 1.4 & 1.4 & 0.3 & 0.9 \\
\hline 75 years and over . . . . . . . . . . . . . & $\ldots$ & $\ldots$ & 1.4 & 1.4 & 0.5 & 0.6 \\
\hline
\end{tabular}

.. Category not applicable.

* Figure does not meet standard of reliability or precision.

NOTE: Numbers may not add to totals because of rounding.

Table 3. Number and percent distribution of emergency department visits with corresponding standard errors, by primary expected source of payment: United States, 2003

\begin{tabular}{|c|c|c|c|c|}
\hline Primary expected source of payment & $\begin{array}{l}\text { Number of } \\
\text { visits in } \\
\text { thousands }\end{array}$ & $\begin{array}{l}\text { Standard } \\
\text { error in } \\
\text { thousands }\end{array}$ & $\begin{array}{c}\text { Percent } \\
\text { distribution }\end{array}$ & $\begin{array}{c}\text { Standard } \\
\text { error of } \\
\text { percent }\end{array}$ \\
\hline All visits $\ldots \ldots \ldots \ldots \ldots \ldots \ldots \ldots \ldots \ldots$ & 113,903 & 4,094 & 100.0 & $\ldots$ \\
\hline 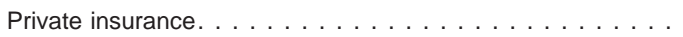 & 41,461 & 1,916 & 36.4 & 0.9 \\
\hline Medicaid or $\mathrm{SCHIP}^{1} \ldots \ldots \ldots \ldots \ldots \ldots \ldots$ & 24,415 & 1,166 & 21.4 & 0.7 \\
\hline Medicare . . . . . . . . . . . . . . . . . . & 18,525 & 753 & 16.3 & 0.4 \\
\hline 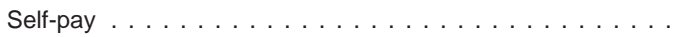 & 16,066 & 849 & 14.1 & 0.6 \\
\hline Worker's compensation . . . . . . . . . . . . . . . . . . . & 2,130 & 164 & 1.9 & 0.1 \\
\hline No charge . . . . . . . . . . . . . . . . . & *1,113 & 423 & *1.0 & 0.4 \\
\hline 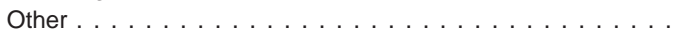 & 2,800 & 367 & 2.5 & 0.3 \\
\hline Unknown or blank . . . . . . . . . . . . . . . . . . & 7,392 & 1,075 & 6.5 & 0.9 \\
\hline
\end{tabular}

.. Category not applicable.

* Figure does not meet standard of reliability or precision.

${ }^{1} \mathrm{SCHIP}$ is State Children's Health Insurance Program.

NOTE: Numbers may not add to totals because of rounding. 


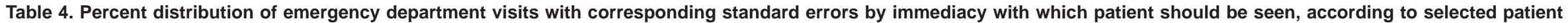
and visit characteristics: United States, 2003

\begin{tabular}{|c|c|c|c|c|c|c|c|c|c|c|c|c|}
\hline Patient and visit characteristics & $\begin{array}{l}\text { Number of } \\
\text { visits in } \\
\text { thousands }\end{array}$ & Total & Emergent ${ }^{1}$ & Urgent $^{2}$ & Semiurgent ${ }^{3}$ & Nonurgent ${ }^{4}$ & $\begin{array}{l}\text { Unknown/ } \\
\text { no triage }\end{array}$ & Emergent ${ }^{1}$ & Urgent $^{2}$ & Semiurgent ${ }^{3}$ & Nonurgent ${ }^{4}$ & $\begin{array}{l}\text { Unknown/ } \\
\text { no triage }{ }^{5}\end{array}$ \\
\hline & & \multicolumn{5}{|c|}{ Percent distribution } & \multicolumn{6}{|c|}{ Standard error of percent } \\
\hline$\ldots \ldots \ldots \ldots$ & 113,903 & 100.0 & 15.2 & 35.2 & 20.0 & 12.8 & 16.7 & 1.1 & 1.7 & 1.3 & 1.4 & 2.0 \\
\hline \multicolumn{13}{|l|}{ Age } \\
\hline Under 15 years $\ldots \ldots \ldots \ldots \ldots \ldots \ldots$ & 24,733 & 100.0 & 10.8 & 33.7 & 22.3 & 15.5 & 17.7 & 1.4 & 2.4 & 1.7 & 2.2 & 2.71 \\
\hline $15-24$ years. . . . . . . . . . . . . . . . . & 17,731 & 100.0 & 11.9 & 34.6 & 22.0 & 15.1 & 16.5 & 1.1 & 1.9 & 1.7 & 1.6 & 2.12 \\
\hline 25-44 years. $\ldots \ldots \ldots \ldots \ldots \ldots \ldots$ & 32,906 & 100.0 & 13.3 & 35.8 & 20.9 & 13.8 & 16.2 & 1.0 & 1.8 & 1.4 & 1.5 & 1.94 \\
\hline $45-64$ years $\ldots \ldots \ldots \ldots \ldots \ldots \ldots$ & 20,992 & 100.0 & 17.7 & 35.7 & 18.3 & 11.5 & 16.9 & 1.2 & 1.8 & 1.4 & 1.3 & 2.06 \\
\hline $65-74$ years. . . . . . . . . . . . . . . & 7,153 & 100.0 & 24.5 & 36.3 & 15.3 & 7.2 & 16.6 & 1.6 & 1.8 & 1.3 & 1.1 & 2.1 \\
\hline 75 years and over $\ldots \ldots \ldots \ldots \ldots$ & 10,389 & 100.0 & 25.5 & 36.5 & 15.5 & 5.9 & 16.7 & 1.7 & 1.7 & 1.3 & 1.1 & 1.9 \\
\hline \multicolumn{13}{|l|}{ Sex } \\
\hline 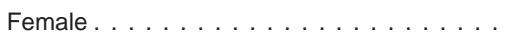 & 60,995 & 100.0 & 14.6 & 35.9 & 20.1 & 13.0 & 16.4 & 1.0 & 1.8 & 1.3 & 1.5 & 1.9 \\
\hline Male $\ldots \ldots \ldots \ldots \ldots \ldots \ldots$ & 52,908 & 100.0 & 15.8 & 34.5 & 20.0 & 12.5 & 17.2 & 1.2 & 1.7 & 1.3 & 1.3 & 2.0 \\
\hline \multicolumn{13}{|l|}{ Race $^{6}$} \\
\hline$\ldots \ldots \ldots$ & 85,833 & 100.0 & 15.4 & 35.7 & 19.9 & 11.9 & 17.0 & 1.1 & 1.9 & 1.5 & 1.3 & 2.1 \\
\hline Black or African American . . . . . . . . & 24,740 & 100.0 & 13.8 & 33.7 & 21.0 & 16.5 & 15.0 & 1.6 & 2.2 & 1.9 & 2.4 & 2.2 \\
\hline Other $\ldots \ldots \ldots \ldots \ldots \ldots \ldots \ldots$ & 3,331 & 100.0 & 18.6 & 34.8 & 17.1 & 7.6 & 22.0 & 3.1 & 2.8 & 2.9 & 1.2 & 4.9 \\
\hline \multicolumn{13}{|l|}{ Expected source of payment } \\
\hline Private insurance $\ldots \ldots \ldots \ldots \ldots \ldots$ & 41,461 & 100.0 & 14.7 & 35.9 & 21.4 & 12.2 & 15.8 & 1.2 & 1.7 & 1.5 & 1.3 & 2.3 \\
\hline Medicaid or $\mathrm{SCHIP}^{7} \ldots \ldots \ldots \ldots \ldots \ldots$ & 24,415 & 100.0 & 12.1 & 34.7 & 21.8 & 15.5 & 15.9 & 1.2 & 2.3 & 1.8 & 2.1 & 2.2 \\
\hline Medicare $\ldots \ldots \ldots \ldots \ldots \ldots \ldots$ & 18,525 & 100.0 & 23.7 & 36.7 & 16.1 & 7.6 & 15.8 & 1.5 & 1.7 & 1.3 & 1.0 & 1.9 \\
\hline Self-pay $\ldots \ldots \ldots \ldots \ldots \ldots \ldots \ldots$ & 16,066 & 100.0 & 13.1 & 35.9 & 21.5 & 15.8 & 13.6 & 1.1 & 2.2 & 1.7 & 1.8 & 2.0 \\
\hline Worker's compensation . . . . . . . . . . . . & 2,130 & 100.0 & 11.0 & 30.8 & 25.7 & 15.6 & 17.0 & 2.0 & 2.8 & 3.2 & 2.8 & 3.2 \\
\hline No charge $\ldots \ldots \ldots \ldots \ldots \ldots \ldots$ & ${ }^{*} 1,113$ & 100.0 & ${ }^{*} 8.6$ & ${ }^{*} 20.7$ & 16.1 & 48.5 & $\ldots$ & 3.5 & 7.4 & 4.2 & 14.3 & $\ldots$ \\
\hline Other $\ldots \ldots \ldots \ldots \ldots \ldots \ldots \ldots$ & 2,800 & 100.0 & 20.8 & 36.1 & 16.5 & 11.7 & 14.8 & 4.4 & 3.7 & 2.3 & 2.2 & 4.2 \\
\hline Unknown or blank . . . . . . . . . . . . & 7,392 & 100.0 & 11.1 & 31.4 & 13.3 & 7.7 & 36.6 & 1.3 & 6.0 & 1.9 & 1.5 & 6.8 \\
\hline
\end{tabular}

.. Category not applicable.

Figure does not meet standard of reliability or precision.

A visit in which the patient should be seen in less than 15 minutes.

A visit in which the patient should be seen within 15-60 minutes.

${ }^{3} A$ visit in which the patient should be seen within $61-120$ minutes.
${ }^{4} \mathrm{~A}$ visit in which the patient should be seen within 121 minutes-24 hours.

${ }^{5} \mathrm{~A}$ visiti in which there is no mention of an immediacy rating or triage level in the medical record or the hospital did not perform triage or the patient was dead on arrival.

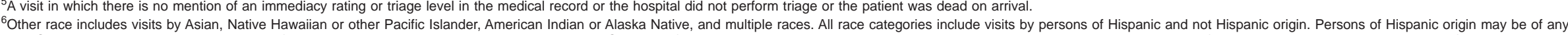

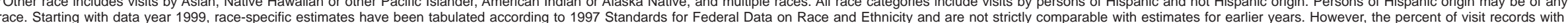
multiple races indicated is small and lower than what is typically found for self-reported race. See "Technical Notes" for more details.

${ }^{7} \mathrm{SCHIP}$ is State Children's Health Insurance Program.

NOTE: Numbers may not add to totals because of rounding. 
Table 5. Number and percent distribution of emergency department visits with corresponding standard errors, by time spent waiting to see a physician and time spent in the emergency department: United States, 2003

\begin{tabular}{|c|c|c|c|c|}
\hline Visit characteristic & $\begin{array}{l}\text { Number of } \\
\text { visits in } \\
\text { thousands }\end{array}$ & $\begin{array}{l}\text { Standard } \\
\text { error in } \\
\text { thousands }\end{array}$ & $\begin{array}{c}\text { Percent } \\
\text { distribution }\end{array}$ & $\begin{array}{c}\text { Standard } \\
\text { error of } \\
\text { percent }\end{array}$ \\
\hline All visits $\ldots \ldots \ldots \ldots \ldots \ldots \ldots$ & 106,633 & 3,811 & 100.0 & $\cdots$ \\
\hline \multicolumn{5}{|l|}{ Time spent waiting to see a physician ${ }^{1}$} \\
\hline Less than 15 minutes . . . . . . . . . . . . . . . . & 22,311 & 1,486 & 20.9 & 1.2 \\
\hline $15-59$ minutes $\ldots \ldots \ldots \ldots \ldots \ldots$ & 42,918 & 2,036 & 40.2 & 1.2 \\
\hline $1-2$ hours $\ldots \ldots \ldots \ldots \ldots \ldots \ldots \ldots$ & 14,612 & 806 & 13.7 & 0.6 \\
\hline $2-3$ hours . . . . . . . . . . . . . . & 4,720 & 345 & 4.4 & 0.3 \\
\hline $3-4$ hours $\ldots \ldots \ldots \ldots \ldots \ldots \ldots \ldots$ & 1,720 & 179 & 1.6 & 0.2 \\
\hline $4-6$ hours . . . . . . . . . . . . . . . . & 1,486 & 213 & 1.4 & 0.2 \\
\hline 6 hours or more . . . . . . . . . . . . . & 121 & 30 & 0.1 & 0.0 \\
\hline Blank . . . . . . . . . . . . . . . . . & 18,745 & 2,113 & 17.6 & 1.9 \\
\hline \multicolumn{5}{|l|}{$\begin{array}{l}\text { Time spent in } \\
\text { the emergency department }\end{array}$} \\
\hline Less than 1 hour $\ldots \ldots \ldots \ldots \ldots \ldots$ & 15,978 & 939 & 14.0 & 0.7 \\
\hline $1-2$ hours $\ldots \ldots \ldots \ldots \ldots \ldots \ldots \ldots$ & 28,656 & 1,203 & 25.2 & 0.5 \\
\hline $2-4$ hours $\ldots \ldots \ldots \ldots \ldots \ldots \ldots \ldots \ldots$ & 35,171 & 1,518 & 30.9 & 0.6 \\
\hline $4-6$ hours . . . . . . . . . . . . . . . . . & 13,039 & 735 & 11.4 & 0.4 \\
\hline $6-10$ hours . . . . . . . . . . . . . . . & 6,456 & 413 & 5.7 & 0.3 \\
\hline $10-14$ hours. . . . . . . . . . . . . . . . . . & 1,565 & 119 & 1.4 & 0.1 \\
\hline 14-23 hours. . . . . . . . . . . . . . . . . . . . & 1,433 & 137 & 1.3 & 0.1 \\
\hline 23-24 hours. . . . . . . . . . . . . . . . . . & 138 & 31 & 0.1 & 0.0 \\
\hline 24 hours or more. . . . . . . . . . . . . . . . & 729 & 147 & 0.6 & 0.1 \\
\hline 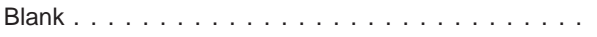 & 10,738 & 1,063 & 9.4 & 0.9 \\
\hline
\end{tabular}

... Category not applicable.

0.0 Quantity more than zero but less than 0.05

${ }^{1}$ Visits where a physician was not seen were excluded.

NOTE: Numbers may not add to totals because of rounding.

Table 6. Number and percent distribution of emergency department visits with corresponding standard errors, by patient's principal reason for visit module: United States, 2003

\begin{tabular}{|c|c|c|c|c|}
\hline Principal reason for visit module and RVC code ${ }^{1}$ & $\begin{array}{l}\text { Number of } \\
\text { visits in } \\
\text { thousands }\end{array}$ & $\begin{array}{c}\text { Standard } \\
\text { error in } \\
\text { thousands }\end{array}$ & $\begin{array}{c}\text { Percent } \\
\text { distribution }\end{array}$ & $\begin{array}{c}\text { Standard } \\
\text { error of } \\
\text { percent }\end{array}$ \\
\hline 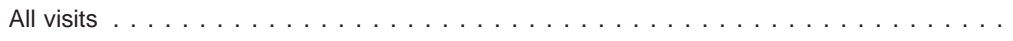 & 113,903 & 4,094 & 100.0 & $\ldots$ \\
\hline Symptom module. . . . . . . . . . . . . . . . . . . . . . . . . . . . . . . . . . S001-s999 & 83,511 & 3,057 & 73.3 & 0.5 \\
\hline General symptoms . . . . . . . . . . . . . . . . . . . . . . . . . . . . . . s001-s099 & 18,473 & 757 & 16.2 & 0.4 \\
\hline Symptoms referable to psychological or mental disorders . . . . . . . . . . S100-S199 & 2,450 & 174 & 2.2 & 0.1 \\
\hline Symptoms referable to the nervous system (excluding sense organs) . . . S200-S259 & 6,857 & 302 & 6.0 & 0.2 \\
\hline Symptoms referable to the cardiovascular or lymphatic system . . . . . . . . S260-S299 & 928 & 86 & 0.8 & 0.1 \\
\hline Symptoms referable to the eyes and ears . . . . . . . . . . . . . . . . S300-S399 & 3,994 & 258 & 3.5 & 0.2 \\
\hline Symptoms referable to the respiratory system . . . . . . . . . . . . . . . . S400-S499 & 13,665 & 597 & 12.0 & 0.3 \\
\hline Symptoms referable to the digestive system . . . . . . . . . . . . . . . . S500-S639 & 15,195 & 669 & 13.3 & 0.3 \\
\hline Symptoms referable to the genitourinary system . . . . . . . . . . . . . . . . . S640-S829 & 4,093 & 242 & 3.6 & 0.2 \\
\hline Symptoms referable to the skin, hair, and nails $\ldots \ldots \ldots \ldots \ldots$. . . . . S830-S899 & 3,204 & 199 & 2.8 & 0.1 \\
\hline Symptoms referable to the musculoskeletal system . . . . . . . . . . . . . . S900-S999 & 14,652 & 613 & 12.9 & 0.3 \\
\hline Disease module . . . . . . . . . . . . . . . . . . . . . . . . . . D001-D999 & 4,258 & 235 & 3.7 & 0.2 \\
\hline Diagnostic, screening, and preventive module . . . . . . . . . . . . . X100-X599 & 781 & 72 & 0.7 & 0.1 \\
\hline Treatment module . . . . . . . . . . . . . . . . . . . . . . . . . . . . . . . . . .T100-T899 & 2,722 & 201 & 2.4 & 0.2 \\
\hline Injuries and adverse effects module . . . . . . . . . . . . . . . . . J001-J999 & 21,530 & 919 & 18.9 & 0.4 \\
\hline Test results module . . . . . . . . . . . . . . . . . . . . . . . . . . . . . . R100-R700 & 315 & 42 & 0.3 & 0.0 \\
\hline Administrative module . . . . . . . . . . . . . . . . . . . . . . . . A100-A140 & 152 & 31 & 0.1 & 0.0 \\
\hline 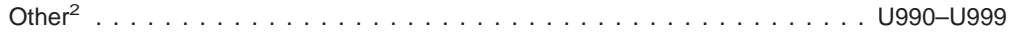 & 635 & 93 & 0.6 & 0.1 \\
\hline
\end{tabular}

Category not applicable

0.0 Quantity more than zero but less than 0.05 .

${ }^{1}$ Based on A Reason for Visit Classification for Ambulatory Care (RVC) (16)

Includes problems and complaints not elsewhere classified, entries of "none," blanks, and illegible entries.

NOTE: Numbers may not add to totals because of rounding. 
Table 7. Number and percent distribution of emergency department visits with corresponding standard errors, by the 20 leading principal reasons for visit by patient: United States, 2003

\begin{tabular}{|c|c|c|c|c|}
\hline Principal reason for visit and RVC code ${ }^{1}$ & $\begin{array}{l}\text { Number of } \\
\text { visits in } \\
\text { thousands }\end{array}$ & $\begin{array}{l}\text { Standard } \\
\text { error in } \\
\text { thousands }\end{array}$ & $\begin{array}{c}\text { Percent } \\
\text { distribution }\end{array}$ & $\begin{array}{c}\text { Standard } \\
\text { error of } \\
\text { percent }\end{array}$ \\
\hline 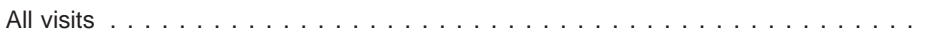 & 113,903 & 4,094 & 100.0 & $\ldots$ \\
\hline Stomach pain, cramps, and spasms . . . . . . . . . . . . . . . . . . . . . S545 & 7,583 & 376 & 6.7 & 0.2 \\
\hline Chest pain and related symptoms (not referable to body system) . . . . . . . . S050 & 5,838 & 259 & 5.1 & 0.2 \\
\hline 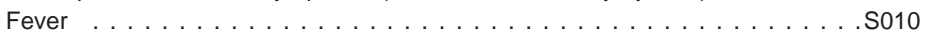 & 5,732 & 441 & 5.0 & 0.3 \\
\hline Cough . . . . . . . . . . . . . . . . . . . . . . . . . . . . . . . . . . . & 3,597 & 223 & 3.2 & 0.1 \\
\hline Headache, pain in head . . . . . . . . . . . . . . . . . . . . . . . . S210 & 3,140 & 167 & 2.8 & 0.1 \\
\hline 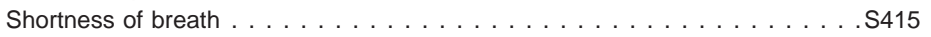 & 2,984 & 210 & 2.6 & 0.2 \\
\hline Back symptoms . . . . . . . . . . . . . . . . . . . . . . . . . . . . s905 & 2,696 & 146 & 2.4 & 0.1 \\
\hline vomiting . . . . . . . . . . . . . . . . . . . . . . . . . . . . . . . . & 2,369 & 180 & 2.1 & 0.1 \\
\hline Symptoms referable to throat . . . . . . . . . . . . . . . . . . . . . . . . S455 & 2,365 & 153 & 2.1 & 0.1 \\
\hline Accident, not otherwise specified . . . . . . . . . . . . . . . . . . . . . J810 & 2,239 & 197 & 2.0 & 0.2 \\
\hline Pain, site not referrable to a specific body system . . . . . . . . . . . . . . S055 & 2,234 & 117 & 2.0 & 0.1 \\
\hline Lacerations and cuts-upper extremity . . . . . . . . . . . . . . . . . . . J J225 & 2,143 & 128 & 1.9 & 0.1 \\
\hline Earache or ear infection . . . . . . . . . . . . . . . . . . . . S355 & 1,867 & 144 & 1.6 & 0.1 \\
\hline Motor vehicle accident, type of injury unspecified $\ldots \ldots$. . . . . . . . . . . . J805 & 1,760 & 131 & 1.5 & 0.1 \\
\hline Skin rash . . . . . . . . . . . . . . . . . . . . . . . . . . . . S860 & 1,688 & 137 & 1.5 & 0.1 \\
\hline Leg symptoms . . . . . . . . . . . . . . . . . . . . . . . . . . . . . . . . . . . . . S920 & 1,615 & 112 & 1.4 & 0.1 \\
\hline Labored or difficult breathing (dyspnea) . . . . . . . . . . . . . . . . . . . . . S420 & 1,584 & 132 & 1.4 & 0.1 \\
\hline Vertigo-dizziness . . . . . . . . . . . . . . . . . . . . . . . . . . . . . . . S225 & 1,503 & 101 & 1.3 & 0.1 \\
\hline Injury, other and unspecified type-head, neck, and face . . . . . . . . . . . . J505 & 1,501 & 113 & 1.3 & 0.1 \\
\hline Nausea . . . . . . . . . . . . . . . . . . . . . . . . . . . . . . . . . . . . . . . . . . . . . . . & 1,408 & 90 & 1.2 & 0.1 \\
\hline 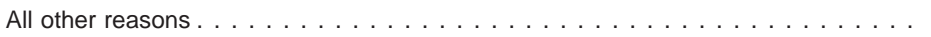 & 58,056 & 2,110 & 51.0 & 0.4 \\
\hline
\end{tabular}

Category not applicable.

${ }^{1}$ Based on A Reason for Visit Classification for Ambulatory Care (RVC) (16).

NOTE: Numbers may not add to totals because of rounding.

Table 8. Number and percent distribution of emergency department visits with corresponding standard errors, by prior emergency department visit: United States, 2003

\begin{tabular}{|c|c|c|c|c|}
\hline Visit characteristic & $\begin{array}{l}\text { Number of } \\
\text { visits in } \\
\text { thousands }\end{array}$ & $\begin{array}{l}\text { Standard } \\
\text { error in } \\
\text { thousands }\end{array}$ & $\begin{array}{c}\text { Percent } \\
\text { distribution }\end{array}$ & $\begin{array}{c}\text { Standard } \\
\text { error of } \\
\text { percent }\end{array}$ \\
\hline 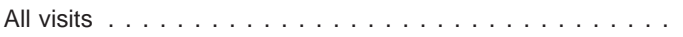 & 113,903 & 4,094 & 100.0 & $\ldots$ \\
\hline \multicolumn{5}{|l|}{ Patient seen in this $E D^{1}$ within the last 72 hours: } \\
\hline Yes. . . . . . . . . . . . . . . . & 3,238 & 224 & 2.8 & 0.2 \\
\hline 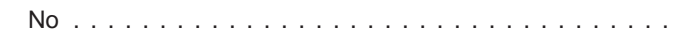 & 101,552 & 3,701 & 89.2 & 1.1 \\
\hline Unknown or blank . . . . . . . . . . . . . . . . & 9,113 & 1,329 & 8.0 & 1.1 \\
\hline \multicolumn{5}{|l|}{ Episode of care: } \\
\hline Initial visit for problem $\ldots \ldots \ldots \ldots \ldots \ldots$ & 101,172 & 3,790 & 88.8 & 0.7 \\
\hline Followup for problem . . . . . . . . . . . . . . . . . & 6,306 & 439 & 5.5 & 0.3 \\
\hline Unknown or blank . . . . . . . . . . . . . . . & 6,425 & 743 & 5.6 & 0.7 \\
\hline
\end{tabular}

\footnotetext{
Category not applicable.
}

${ }^{1} E D$ is emergency department.

NOTE: Numbers may not add to totals because of rounding. 
Table 9. Number and percent distribution of emergency department visits with corresponding standard errors, by primary diagnosis: United States, 2003

\begin{tabular}{|c|c|c|c|c|}
\hline Major disease category and ICD-9-CM code range ${ }^{1}$ & $\begin{array}{l}\text { Number of } \\
\text { visits in } \\
\text { thousands }\end{array}$ & $\begin{array}{l}\text { Standard } \\
\text { error in } \\
\text { thousands }\end{array}$ & $\begin{array}{c}\text { Percent } \\
\text { distribution }\end{array}$ & $\begin{array}{c}\text { Standard } \\
\text { error of } \\
\text { percent }\end{array}$ \\
\hline 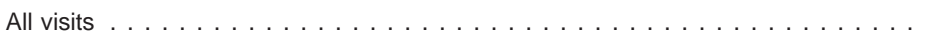 & 113,903 & 4,094 & 100.0 & $\cdots$ \\
\hline Infectious and parasitic diseases . . . . . . . . . . . . . . . . . . 001-139 & 3,342 & 181 & 2.9 & 0.1 \\
\hline Neoplasms . . . . . . . . . . . . . . . . . . . . . . . . . . . . . . . . . . . . . . . 140-239 & 300 & 42 & 0.3 & 0.0 \\
\hline Endocrine, nutritional, metabolic diseases, and immunity disorders . . . . . 240-279 & 1,766 & 127 & 1.6 & 0.1 \\
\hline Mental disorders . . . . . . . . . . . . . . . . . . . . . . . . . . . . . . . . . 290-319 & 3,718 & 212 & 3.3 & 0.2 \\
\hline Diseases of the nervous system and sense organs . . . . . . . . . . . 320-389 & 6,191 & 361 & 5.4 & 0.2 \\
\hline Diseases of the circulatory system . . . . . . . . . . . . . . . 390-459 & 4,497 & 240 & 3.9 & 0.2 \\
\hline Diseases of the respiratory system . . . . . . . . . . . . . . . . . . 460-519 & 14,012 & 643 & 12.3 & 0.3 \\
\hline Diseases of the digestive system . . . . . . . . . . . . . . . . . . . 520-579 & 6,611 & 333 & 5.8 & 0.2 \\
\hline Diseases of the genitourinary system . . . . . . . . . . . . . . . . . . . 580-629 & 5,048 & 227 & 4.4 & 0.1 \\
\hline Diseases of the skin and subcutaneous tissue . . . . . . . . . . . . . .680-709 & 3,769 & 241 & 3.3 & 0.2 \\
\hline Diseases of the musculoskeletal system and connective tissue . . . . . . 710-739 & 6,110 & 292 & 5.4 & 0.2 \\
\hline Symptoms, signs, and ill-defined conditions . . . . . . . . . . . . . . . . 780-799 & 21,324 & 909 & 18.7 & 0.5 \\
\hline Injury and poisoning . . . . . . . . . . . . . . . . . . . . . . 800-999 & 29,181 & 1,171 & 25.6 & 0.4 \\
\hline Fractures . . . . . . . . . . . . . . . . . . . . . . . . . . . . . . 800-829 & 3,701 & 214 & 3.2 & 0.1 \\
\hline Sprains and strains . . . . . . . . . . . . . . . . . . . . . . . . . . . . 840-848 & 6,074 & 304 & 5.3 & 0.2 \\
\hline Intracranial injury . . . . . . . . . . . . . . . . . . . . . . . . . 850-854 & 291 & 37 & 0.3 & 0.0 \\
\hline Open wounds . . . . . . . . . . . . . . . . . . . . . . . . . . . . . . . . . . . 870-897 & 6,442 & 308 & 5.7 & 0.2 \\
\hline Superficial injury . . . . . . . . . . . . . . . . . . . . . . . . . . . . . . . . 910-919 & 1,817 & 155 & 1.6 & 0.1 \\
\hline Contusion with intact skin surface . . . . . . . . . . . . . . . . . . . . . . 920-924 & 4,831 & 239 & 4.2 & 0.2 \\
\hline Foreign bodies . . . . . . . . . . . . . . . . . . . . . . . . . . . . . . . 9 930-939 & 640 & 68 & 0.6 & 0.1 \\
\hline Burns . . . . . . . . . . . . . . . . . . . . . . . . . . . . . . 940-949 & 511 & 52 & 0.4 & 0.0 \\
\hline Trauma complications and unspecified injuries . . . . . . . . . . . . . . . 958-959 & 1,737 & 143 & 1.5 & 0.1 \\
\hline Poisoning and toxic effects . . . . . . . . . . . . . . . . . . . . . . . . 960-989 & 1,009 & 79 & 0.9 & 0.1 \\
\hline Surgical and medical complications . . . . . . . . . . . . . . . . . . . . 996-999 & 511 & 57 & 0.4 & 0.0 \\
\hline Other injuries. . . . . . . . . . . . . . . . . . . . . . . . . . . & 1,617 & 103 & 1.4 & 0.1 \\
\hline 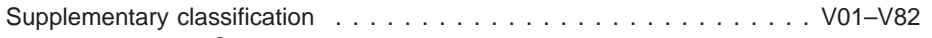 & 2,762 & 178 & 2.4 & 0.1 \\
\hline 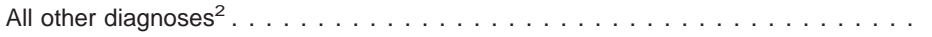 & 3,389 & 264 & 3.0 & 0.2 \\
\hline 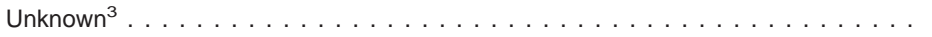 & 1,884 & 184 & 1.7 & 0.2 \\
\hline
\end{tabular}

.. Category not applicable.

0.0 Quantity more than zero but less than 0.05 .

${ }^{1}$ Based on the International Classification of Diseases, 9th Revision, Clinical Modification (ICD-9-CM) (13).

${ }^{2}$ Includes diseases of the blood and blood-forming organs (280-289); complications of pregnancy, childbirth, and the puerperium (630-677); congenital anomalies (740-759); certain conditions originating in the perinatal period (760-779), diagnoses that were uncodable or illegible, patient left before being seen, patient was transferred to another facility, health maintenance organization did not authorize treatment, and entries of "none," "no diagnosis," "no disease," or "healthy."

${ }^{3}$ Includes blank diagnoses.

NOTE: Numbers may not add to totals because of rounding. 
Table 10. Number and percent distribution of emergency department visits with corresponding standard errors, by the 20 leading diagnosis groups: United States, 2003

\begin{tabular}{|c|c|c|c|c|}
\hline Primary diagnosis group and ICD-9-CM code $(\mathrm{s})^{1}$ & $\begin{array}{l}\text { Number of } \\
\text { visits in } \\
\text { thousands }\end{array}$ & $\begin{array}{l}\text { Standard } \\
\text { error in } \\
\text { thousands }\end{array}$ & $\begin{array}{c}\text { Percent } \\
\text { distribution }\end{array}$ & $\begin{array}{c}\text { Standard } \\
\text { error of } \\
\text { percent }\end{array}$ \\
\hline 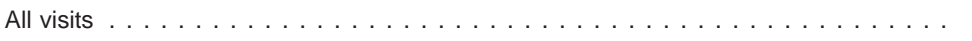 & 113,903 & 4,094 & 100.0 & $\ldots$ \\
\hline Contusion with intact skin surface . . . . . . . . . . . . . . . . . . . . . 920-924 & 4,831 & 239 & 4.2 & 0.2 \\
\hline Acute upper respiratory infections, excluding pharyngitis . . . . . . 460-461,463-466 & 4,584 & 268 & 4.0 & 0.2 \\
\hline Abdominal pain . . . . . . . . . . . . . . . . . . . . 789 & 4,498 & 249 & 3.9 & 0.2 \\
\hline 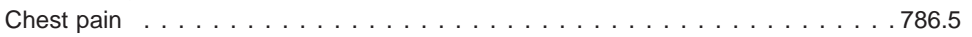 & 4,222 & 214 & 3.7 & 0.1 \\
\hline Open wound, excluding head . . . . . . . . . . . . . . . . . . . . . . . 874-897 & 4,145 & 214 & 3.6 & 0.1 \\
\hline Spinal disorders . . . . . . . . . . . . . . . . . . . . . . . . . . . . . 720-724 & 2,822 & 138 & 2.5 & 0.1 \\
\hline Otitis media and eustachian tube disorders. . . . . . . . . . . . . . . . . . 381-382 & 2,603 & 211 & 2.3 & 0.1 \\
\hline Sprains and strains, excluding ankle and back. . . . . . . . . . . . . 840-844,845.1,848 & 2,553 & 165 & 2.2 & 0.1 \\
\hline Fractures, excluding lower limb . . . . . . . . . . . . . . . . . . . . . . . . . 800-819 & 2,387 & 146 & 2.1 & 0.1 \\
\hline Open wound of head . . . . . . . . . . . . . . . . . . . . . . . . 870-873 & 2,298 & 136 & 2.0 & 0.1 \\
\hline Sprains and strains of neck and back . . . . . . . . . . . . . . . . . . 846,847 & 2,275 & 140 & 2.0 & 0.1 \\
\hline 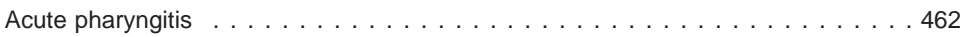 & 1,953 & 136 & 1.7 & 0.1 \\
\hline Urinary tract infection, site not specified . . . . . . . . . . . . . . . . . . . . 599 & 1,860 & 132 & 1.6 & 0.1 \\
\hline Chronic and unspecified bronchitis . . . . . . . . . . . . . . . . . . . . . . 490-491 & 1,835 & 143 & 1.6 & 0.1 \\
\hline Superficial injuries . . . . . . . . . . . . . . . . . . . . . . . . . . . . . . . 910-919 & 1,817 & 155 & 1.6 & 0.1 \\
\hline Cellulitis and abscess $\ldots \ldots \ldots \ldots \ldots \ldots$. . . . . . . . . . . . . . . . . . . . . . . . & 1,810 & 157 & 1.6 & 0.1 \\
\hline 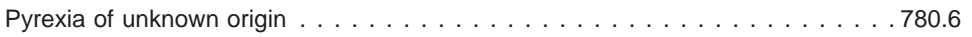 & 1,758 & 165 & 1.5 & 0.1 \\
\hline Asthma . . . . . . . . . . . . . . . . . . . . . . . . . .493 & 1,753 & 123 & 1.5 & 0.1 \\
\hline Heart disease, excluding ischemic . . . .391-392.0,393-398,402,404,415-416,420-429 & 1,743 & 116 & 1.5 & 0.1 \\
\hline Rheumatism, excluding back . . . . . . . . . . . . . . . . . . . . . . . 725-729 & 1,723 & 127 & 1.5 & 0.1 \\
\hline 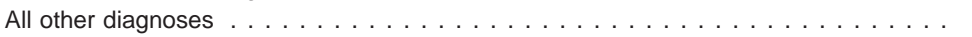 & 60,434 & 2,116 & 53.1 & 0.4 \\
\hline
\end{tabular}

Category not applicable.

${ }^{1}$ Based on the International Classification of Diseases, 9th Revision, Clinical Modification (ICD-9-CM) (17). However, certain codes have been combined in this table to describe the utilization of ambulatory care services.

NOTE: Numbers may not add to totals because of rounding. 
Table 11. Number and percent distribution of emergency department visits with corresponding standard errors by patient's age, according to the five leading primary diagnosis groups: United States, 2003

\begin{tabular}{|c|c|c|c|c|c|c|}
\hline Primary diagnosis group and ICD-9-CM code $(\mathrm{s})^{1}$ & $\begin{array}{l}\text { Number of } \\
\text { visits in } \\
\text { thousands }\end{array}$ & $\begin{array}{l}\text { Standard } \\
\text { error in } \\
\text { thousands }\end{array}$ & $\begin{array}{c}\text { Percent } \\
\text { distribution }\end{array}$ & $\begin{array}{l}\text { Standard } \\
\text { error of } \\
\text { percent }\end{array}$ & $\begin{array}{l}\text { Number of } \\
\text { visits per } \\
100 \text { persons } \\
\text { per year }^{2}\end{array}$ & $\begin{array}{c}\text { Standard } \\
\text { error of } \\
\text { rate }\end{array}$ \\
\hline 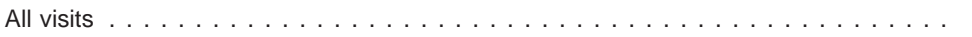 & 113,903 & 4,094 & 100.0 & $\ldots$ & 39.9 & 1.4 \\
\hline \multicolumn{7}{|l|}{ Under 1 year } \\
\hline 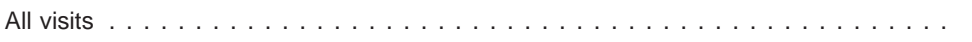 & 3,899 & 315 & 100.0 & $\ldots$ & 97.5 & 7.9 \\
\hline Acute upper respiratory infections, excluding pharyngitis . . . . . . .460-461,463-466 & 715 & 77 & 18.3 & 1.4 & 17.9 & 1.9 \\
\hline Otitis media and eustachian tube disorders . . . . . . . . . . . . . . . . 381-382 & 415 & 62 & 10.6 & 1.3 & 10.4 & 1.6 \\
\hline 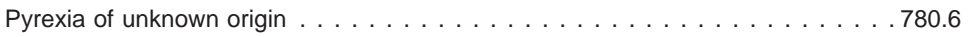 & 289 & 41 & 7.4 & 0.8 & 7.2 & 1.0 \\
\hline Unspecified viral and chlamydial infections . . . . . . . . . . . . . . . . . . . 079.9 & 178 & 27 & 4.6 & 0.7 & 4.5 & 0.7 \\
\hline Noninfectious enteritis and colitis . . . . . . . . . . . . . . . . . . . . . . . 555-558 & 127 & 29 & 3.3 & 0.6 & 3.2 & 0.7 \\
\hline 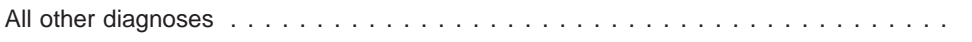 & 2,174 & 190 & 55.8 & 1.9 & 54.3 & 4.7 \\
\hline \multicolumn{7}{|l|}{$1-12$ years } \\
\hline 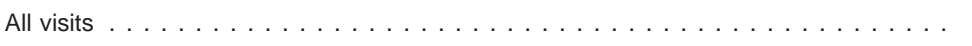 & 18,517 & 1,141 & 100.0 & $\ldots$ & 38.4 & 2.4 \\
\hline Acute upper respiratory infection, excluding pharyngitis . . . . . . . . 460-461,463-466 & 1,826 & 148 & 9.9 & 0.6 & 3.8 & 0.3 \\
\hline Otitis media and eustachian tube disorders. . . . . . . . . . . . . . . . . . . 381-382 & 1,583 & 153 & 8.5 & 0.5 & 3.3 & 0.3 \\
\hline 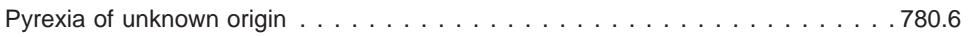 & 960 & 118 & 5.2 & 0.5 & 2.0 & 0.2 \\
\hline Open wound of head . . . . . . . . . . . . . . . . . . . . . . . . 870-873 & 882 & 83 & 4.8 & 0.4 & 1.8 & 0.2 \\
\hline Contusion with intact skin surface . . . . . . . . . . . . . . . . . . . . . . 920-924 & 880 & 81 & 4.8 & 0.4 & 1.8 & 0.2 \\
\hline 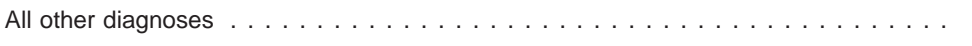 & 12,385 & 799 & 66.8 & 1.0 & 25.7 & 1.7 \\
\hline \multicolumn{7}{|l|}{ 13-21 years } \\
\hline 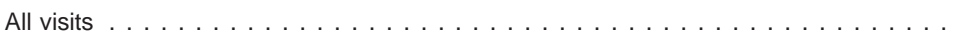 & 14,098 & 584 & 100.0 & $\cdots$ & 38.5 & 1.6 \\
\hline Contusion with intact skin surface . . . . . . . . . . . . . . . . . . . . . . . 920-924 & 948 & 68 & 6.7 & 0.4 & 2.6 & 0.2 \\
\hline Abdominal pain . . . . . . . . . . . . . . . . . . . . . . . . . . . . . 789.0 & 615 & 67 & 4.4 & 0.4 & 1.7 & 0.2 \\
\hline Open wound, excluding head . . . . . . . . . . . . . . . . . . . . . . . . . 874-897 & 598 & 51 & 4.2 & 0.4 & 1.6 & 0.1 \\
\hline Sprains and strains, excluding ankles and back . . . . . . . . . 840-844,845.1,848 & 533 & 81 & 3.8 & 0.5 & 1.5 & 0.2 \\
\hline Complications of pregnancy, childbirth, and the puerperium. . . . . . . . . . . 630-677 & 500 & 67 & 3.5 & 0.4 & $2.8^{3}$ & 0.4 \\
\hline 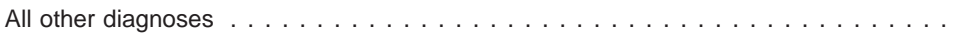 & 10,903 & 461 & 77.3 & 0.9 & 29.8 & 1.3 \\
\hline \multicolumn{7}{|l|}{$22-49$ years } \\
\hline 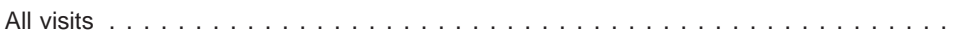 & 45,895 & 1,755 & 100.0 & $\ldots$ & 39.6 & 1.5 \\
\hline 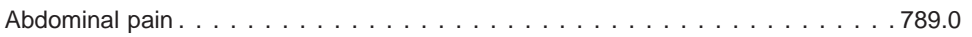 & 2,331 & 155 & 5.1 & 0.3 & 2.0 & 0.1 \\
\hline Open wound, excluding head . . . . . . . . . . . . . . . . . . . . . . . . 874-897 & 2,068 & 152 & 4.5 & 0.3 & 1.8 & 0.1 \\
\hline 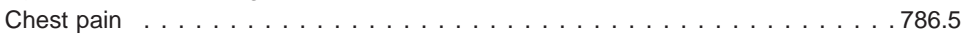 & 1,908 & 117 & 4.2 & 0.2 & 1.7 & 0.1 \\
\hline Contusion with intact skin surface . . . . . . . . . . . . . . . . . . . . . . . . 920-924 & 1,814 & 128 & 4.0 & 0.2 & 1.6 & 0.1 \\
\hline Spinal disorders . . . . . . . . . . . . . . . . . . . . . . . . . . . . . . . . 720-724 & 1,683 & 104 & 3.7 & 0.2 & 1.5 & 0.1 \\
\hline 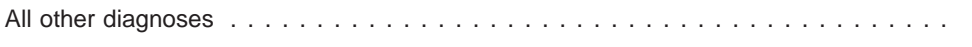 & 36,091 & 1,372 & 78.6 & 0.4 & 31.2 & 1.2 \\
\hline \multicolumn{7}{|l|}{$50-64$ years } \\
\hline 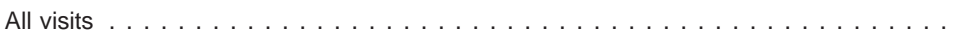 & 13,953 & 560 & 100.0 & $\ldots$ & 29.9 & 1.2 \\
\hline 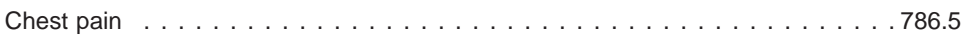 & 951 & 79 & 6.8 & 0.5 & 2.0 & 0.2 \\
\hline Abdominal pain . . . . . . . . . . . . . . . . . . . . . . . . . . . 789.0 & 540 & 56 & 3.9 & 0.4 & 1.2 & 0.1 \\
\hline Contusion with intact skin surface . . . . . . . . . . . . . . . . . . . . . . . . 920-924 & 532 & 56 & 3.8 & 0.4 & 1.1 & 0.1 \\
\hline Spinal disorders . . . . . . . . . . . . . . . . . . . . . . . . . . . . . . . . . .720-724 & 510 & 46 & 3.7 & 0.3 & 1.1 & 0.1 \\
\hline Open wound, excluding head . . . . . . . . . . . . . . . . . . . . . . . . . . . . 874-897 & 430 & 38 & 3.1 & 0.3 & 0.9 & 0.1 \\
\hline 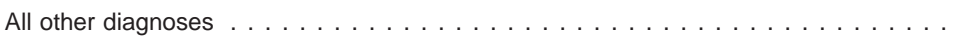 & 10,989 & 464 & 78.8 & 0.8 & 23.6 & 1.0 \\
\hline \multicolumn{7}{|l|}{65 years and over } \\
\hline 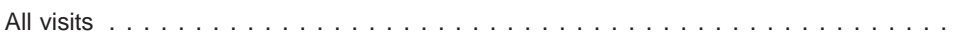 & 17,542 & 754 & 100.0 & $\cdots$ & 51.2 & 2.2 \\
\hline Heart disease, excluding ischemic . . . . 391-392.0,393-398,402,404,415-416,420-429 & 1,035 & 78 & 5.9 & 0.4 & 3.0 & 0.2 \\
\hline 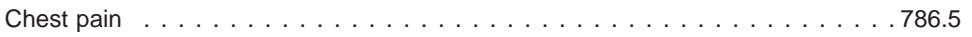 & 1,032 & 92 & 5.9 & 0.5 & 3.0 & 0.3 \\
\hline Pneumonia . . . . . . . . . . . . . . . . . . . . . . . . . . . . . . . 480-486 & 662 & 76 & 3.8 & 0.4 & 1.9 & 0.2 \\
\hline Abdominal pain . . . . . . . . . . . . . . . . . . . . . . . . . . . . 789.0 & 614 & 60 & 3.5 & 0.3 & 1.8 & 0.2 \\
\hline Contusion with intact skin surface . . . . . . . . . . . . . . . . . . . . . . . . 920-924 & 599 & 53 & 3.4 & 0.3 & 1.8 & 0.2 \\
\hline 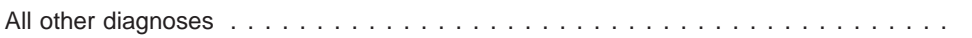 & 13,599 & 605 & 77.5 & 0.8 & 39.7 & 1.8 \\
\hline
\end{tabular}

Category not applicable.

${ }^{1}$ Based on the International Classification of Diseases, 9th Revision, Clinical Modification (ICD-9-CM) (17). However, certain codes have been combined in this table to describe the ulitization of ambulatory care services.

${ }^{2}$ Visit rates by age are based on the July 1, 2003, set of estimates of the civilian noninstitutional population of the United States as developed by the Population Division, U.S. Census Bureau. These population estimates reflect Census 2000 data and are available from the U.S. Census Bureau. See "Technical Notes" for more details.

${ }^{3}$ The population used for the rate is based on visits by females $13-21$ years of age.

NOTE: Numbers may not add to totals because of rounding. 
Table 12. Number, percent distribution, and annual rate of injury-related emergency department visits with corresponding standard errors, by patient's age, sex, and race: United States, 2003

\begin{tabular}{|c|c|c|c|c|c|c|}
\hline Patient's age, sex, and race & $\begin{array}{l}\text { Number of } \\
\text { visits in } \\
\text { thousands }\end{array}$ & $\begin{array}{l}\text { Standard } \\
\text { error in } \\
\text { thousands }\end{array}$ & $\begin{array}{c}\text { Percent } \\
\text { distribution }\end{array}$ & $\begin{array}{l}\text { Standard } \\
\text { error of } \\
\text { percent }\end{array}$ & $\begin{array}{l}\text { Number of } \\
\text { visits per } \\
100 \text { persons } \\
\text { per year }^{1,2}\end{array}$ & $\begin{array}{c}\text { Standard } \\
\text { error of } \\
\text { rate }\end{array}$ \\
\hline All injury-related visits . . . . . . . . . . . . . . . . & 40,194 & 1,534 & 100.0 & $\ldots$ & 14.1 & 0.5 \\
\hline \multicolumn{7}{|l|}{ Age } \\
\hline Under 15 years . . . . . . . . . . . . . . . . . & 8,276 & 493 & 20.6 & 0.9 & 13.6 & 0.8 \\
\hline 15-24 years . . . . . . . . . . . . . . . . . . & 7,222 & 313 & 18.0 & 0.4 & 18.0 & 0.8 \\
\hline 25-44 years . . . . . . . . . . . . . . . . & 12,771 & 552 & 31.8 & 0.6 & 15.5 & 0.7 \\
\hline $45-64$ years . . . . . . . . . . . . . . . . . & 7,276 & 355 & 18.1 & 0.5 & 10.7 & 0.5 \\
\hline $65-74$ years . . . . . . . . . . . . . . . . . . & 1,966 & 127 & 4.9 & 0.3 & 10.9 & 0.7 \\
\hline 75 years and over $\ldots \ldots \ldots \ldots \ldots \ldots$ & 2,682 & 153 & 6.7 & 0.4 & 16.6 & 0.9 \\
\hline \multicolumn{7}{|l|}{ Sex and age } \\
\hline Female . . . . . . . . . . . . . . . . . & 18,533 & 706 & 46.1 & 0.4 & 12.7 & 0.5 \\
\hline Under 15 years $\ldots \ldots \ldots \ldots \ldots \ldots$ & 3,483 & 233 & 8.7 & 0.4 & 11.8 & 0.8 \\
\hline 15-24 years . . . . . . . . . . . . . . & 3,275 & 169 & 8.1 & 0.3 & 16.5 & 0.8 \\
\hline $25-44$ years $\ldots \ldots \ldots \ldots \ldots \ldots \ldots$ & 5,629 & 268 & 14.0 & 0.4 & 13.5 & 0.6 \\
\hline $45-64$ years $\ldots \ldots \ldots \ldots \ldots \ldots \ldots$ & 3,460 & 187 & 8.6 & 0.3 & 9.9 & 0.5 \\
\hline $65-74$ years . . . . . . . . . . . . . . . & 1,045 & 80 & 2.6 & 0.2 & 10.6 & 0.8 \\
\hline 75 years and over . . . . . . . . . . . . & 1,641 & 105 & 4.1 & 0.3 & 16.5 & 1.1 \\
\hline Male ....................... & 21,661 & 868 & 53.9 & 0.4 & 15.5 & 0.6 \\
\hline Under 15 years $\ldots \ldots \ldots \ldots \ldots \ldots \ldots$ & 4,793 & 286 & 11.9 & 0.5 & 15.4 & 0.9 \\
\hline 15-24 years . . . . . . . . . . . . . . & 3,948 & 185 & 9.8 & 0.3 & 19.5 & 0.9 \\
\hline $25-44$ years . . . . . . . . . . . . . . . . & 7,142 & 330 & 17.8 & 0.4 & 17.6 & 0.8 \\
\hline $45-64$ years $\ldots \ldots \ldots \ldots \ldots \ldots \ldots$ & 3,816 & 215 & 9.5 & 0.3 & 11.5 & 0.6 \\
\hline $65-74$ years . . . . . . . . . . . . . . . & 920 & 75 & 2.3 & 0.2 & 11.2 & 0.9 \\
\hline 75 years and over $\ldots \ldots \ldots \ldots \ldots$ & 1,042 & 86 & 2.6 & 0.2 & 16.6 & 1.4 \\
\hline \multicolumn{7}{|l|}{ Race and age $^{3}$} \\
\hline White . . . . . . . . . . . . . . . & 31,570 & 1,414 & 78.5 & 1.3 & 13.7 & 0.6 \\
\hline Under 15 years $\ldots \ldots \ldots \ldots \ldots \ldots \ldots$ & 6,416 & 424 & 16.0 & 0.8 & 13.8 & 0.9 \\
\hline 15-24 years . . . . . . . . . . . . . . . . & 5,566 & 286 & 13.8 & 0.4 & 17.8 & 0.9 \\
\hline 25-44 years . . . . . . . . . . . . . . . & 9,794 & 495 & 24.4 & 0.6 & 14.9 & 0.8 \\
\hline $45-64$ years $\ldots \ldots \ldots \ldots \ldots \ldots \ldots$ & 5,817 & 324 & 14.5 & 0.5 & 10.2 & 0.6 \\
\hline $65-74$ years $\ldots \ldots \ldots \ldots \ldots \ldots \ldots$ & 1,604 & 107 & 4.0 & 0.2 & 10.3 & 0.7 \\
\hline 75 years and over. . . . . . . . . . . . . & 2,373 & 144 & 5.9 & 0.3 & 16.4 & 1.0 \\
\hline Black or African American $\ldots \ldots \ldots \ldots$ & 7,487 & 502 & 18.6 & 1.2 & 21.0 & 1.4 \\
\hline Under 15 years $\ldots \ldots \ldots \ldots \ldots \ldots \ldots$ & 1,579 & 161 & 3.9 & 0.4 & 16.7 & 1.7 \\
\hline $15-24$ years $\ldots \ldots \ldots \ldots \ldots \ldots \ldots \ldots$ & 1,467 & 131 & 3.6 & 0.3 & 25.3 & 2.3 \\
\hline 25-44 years . . . . . . . . . . . . . . . & 2,597 & 193 & 6.5 & 0.5 & 25.1 & 1.9 \\
\hline $45-64$ years $\ldots \ldots \ldots \ldots \ldots \ldots \ldots \ldots$ & 1,290 & 117 & 3.2 & 0.3 & 17.6 & 1.6 \\
\hline $65-74$ years $\ldots \ldots \ldots \ldots \ldots \ldots \ldots$ & 306 & 47 & 0.8 & 0.1 & 18.5 & 2.8 \\
\hline 75 years and over. . . . . . . . . . . . . . . . & 250 & 40 & 0.6 & 0.1 & 20.8 & 3.3 \\
\hline Other $\ldots \ldots \ldots \ldots \ldots \ldots \ldots \ldots \ldots$ & 1,136 & 167 & 2.8 & 0.4 & 5.9 & 0.9 \\
\hline \multicolumn{7}{|l|}{ Hospital characteristics } \\
\hline \multicolumn{7}{|l|}{ Ownership: } \\
\hline Voluntary . . . . . . . . . . . . . . . . . . . & 29,306 & 1,624 & 72.9 & 2.5 & 10.3 & 0.6 \\
\hline Government . . . . . . . . . . . . . . . . & 7,327 & 937 & 18.2 & 2.3 & 2.6 & 0.3 \\
\hline Proprietary . . . . . . . . . . . . . . . . . . & 3,560 & 540 & 8.9 & 1.4 & 1.3 & 0.2 \\
\hline \multicolumn{7}{|l|}{ Geographic region: } \\
\hline Northeast . . . . . . . . . . . . . . . . . . . . & 8,874 & 691 & 22.1 & 1.6 & 16.6 & 1.9 \\
\hline Midwest . . . . . . . . . . . . . . . . . & 8,937 & 591 & 22.2 & 1.4 & 13.9 & 1.3 \\
\hline South $\ldots \ldots \ldots \ldots \ldots \ldots \ldots \ldots$ & 15,071 & 932 & 37.5 & 1.9 & 14.7 & 1.3 \\
\hline 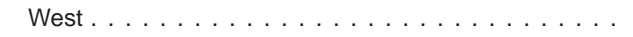 & 7,312 & 858 & 18.2 & 1.8 & 11.2 & 1.2 \\
\hline \multicolumn{7}{|l|}{ Metropolitan status: } \\
\hline 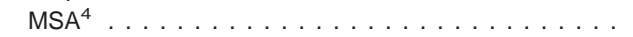 & 32,145 & 1,445 & 80.0 & 2.3 & 13.5 & 0.6 \\
\hline Non-MSA $^{4} \ldots \ldots \ldots \ldots \ldots \ldots \ldots \ldots$ & 8,048 & 1,026 & 20.0 & 2.3 & 17.1 & 2.2 \\
\hline
\end{tabular}

Category not applicable.

${ }^{1}$ Visit rates for age, sex, race and region are based on the July 1, 2003, set of estimates of the civilian noninstitutional population of the United States as developed by the Population Division, U.S. Census Bureau. These population estimates reflect Census 2000 data and are available from the Census Bureau. See "Technical Notes" for more details.

${ }^{2}$ Population estimates of metropolitan statistical area status are based on data from the 2003 National Health Interview Survey, National Center for Health Statistics, adjusted to the U.S. Census Bureau definition of core-based statistical areas as of December 2003. See www.census.gov/population/www/estimates/metrodef.html for more about metropolitan statistical area definitions.

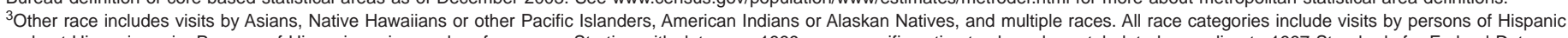

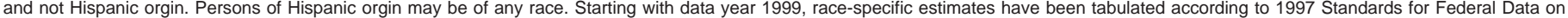
Race and Ethnicity and are not strictly comparable with estimates for earlier years. However, the percentage of visit records with multiple races indicated is small and lower than what is typically found for self-reported race. See "Technical Notes" for more details.

${ }^{4} \mathrm{MSA}$ is metropolitan statistical area.

NOTE: Numbers may not add to totals because of rounding. 
Table 13. Number and percent distribution of injury-related emergency department visits with corresponding standard errors, by intent and mechanism of external cause: United States, 2003

\begin{tabular}{|c|c|c|c|c|}
\hline Intent and mechanism ${ }^{1}$ & $\begin{array}{l}\text { Number of } \\
\text { visits in } \\
\text { thousands }\end{array}$ & $\begin{array}{l}\text { Standard } \\
\text { error in } \\
\text { thousands }\end{array}$ & $\begin{array}{c}\text { Percent } \\
\text { distribution }\end{array}$ & $\begin{array}{c}\text { Standard } \\
\text { error of } \\
\text { percent }\end{array}$ \\
\hline 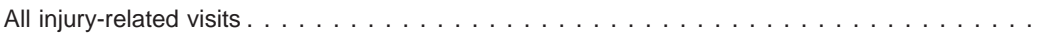 & 40,194 & 1,534 & 100.0 & $\ldots$ \\
\hline 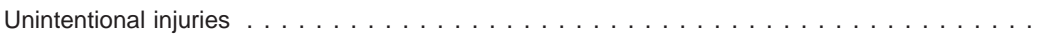 & 28,047 & 1,188 & 69.8 & 0.8 \\
\hline 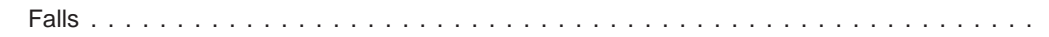 & 7,989 & 375 & 19.9 & 0.6 \\
\hline Struck against or struck accidentally by objects or persons $\ldots \ldots \ldots \ldots \ldots \ldots$ & 4,209 & 241 & 10.5 & 0.4 \\
\hline 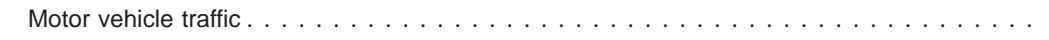 & 4,161 & 207 & 10.4 & 0.3 \\
\hline 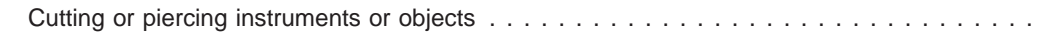 & 2,544 & 163 & 6.3 & 0.3 \\
\hline 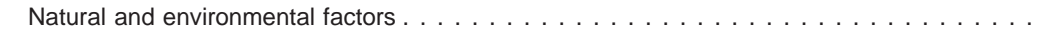 & 2,023 & 162 & 5.0 & 0.3 \\
\hline 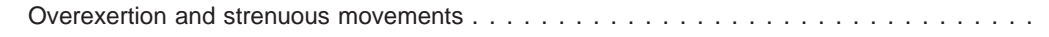 & 1,686 & 100 & 4.2 & 0.2 \\
\hline 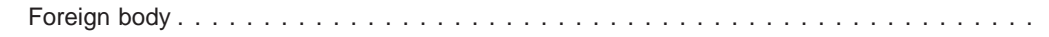 & 836 & 80 & 2.1 & 0.2 \\
\hline Poisoning . . . . . . . . . . . . . . . . . . . . . . . . . . . & 750 & 60 & 1.9 & 0.1 \\
\hline Fire and flames, hot substances or object, caustic or corrosive material and steam $\ldots \ldots$ & 516 & 47 & 1.3 & 0.1 \\
\hline Caught accidentally in or between objects $\ldots \ldots \ldots \ldots \ldots \ldots \ldots \ldots \ldots \ldots \ldots \ldots \ldots$ & 480 & 62 & 1.2 & 0.1 \\
\hline Pedal cycle, nontraffic . . . . . . . . . . . . . . . . . . . . . . . . . . . & 459 & 48 & 1.1 & 0.1 \\
\hline 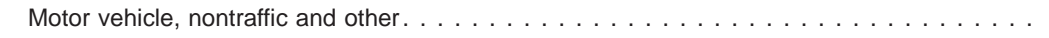 & 421 & 73 & 1.0 & 0.2 \\
\hline Machinery. . . . . . . . . . . . . . . . . . . . . . . . . . . & 328 & 54 & 0.8 & 0.1 \\
\hline 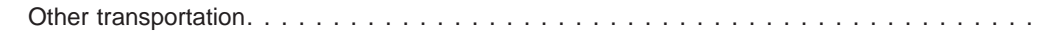 & 190 & 31 & 0.5 & 0.1 \\
\hline 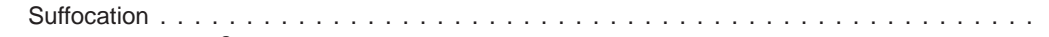 & 122 & 32 & 0.3 & 0.1 \\
\hline 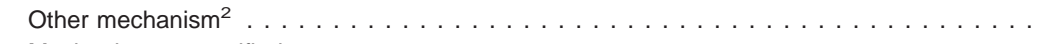 & 662 & 71 & 1.6 & 0.2 \\
\hline Mechanism unspecified $\ldots \ldots \ldots \ldots \ldots \ldots \ldots \ldots \ldots \ldots \ldots$ & 672 & 70 & 1.7 & 0.2 \\
\hline 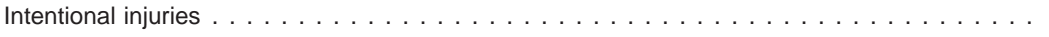 & 2,086 & 129 & 5.2 & 0.3 \\
\hline 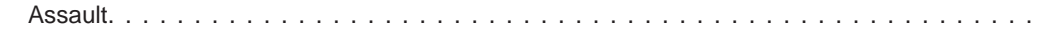 & 1,608 & 107 & 4.0 & 0.2 \\
\hline Unarmed fight or brawl, striking by blunt or thrown object $\ldots \ldots \ldots \ldots \ldots \ldots$ & 1,054 & 88 & 2.6 & 0.2 \\
\hline 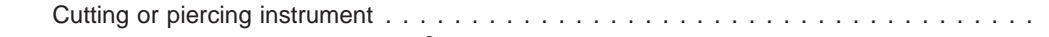 & 91 & 16 & 0.2 & 0.0 \\
\hline 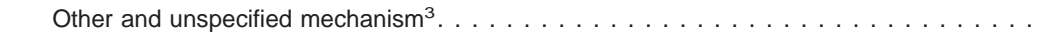 & 462 & 47 & 1.2 & 0.1 \\
\hline 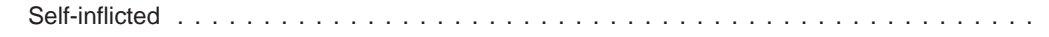 & 438 & 54 & 1.1 & 0.1 \\
\hline Poisoning by solid or liquid substances, gases, and vapors . . . . . . . . . . . . . & 313 & 50 & 0.8 & 0.1 \\
\hline Other and unspecified mechanism ${ }^{4} \ldots \ldots \ldots \ldots \ldots \ldots \ldots \ldots \ldots$ & 124 & 20 & 0.3 & 0.1 \\
\hline Other causes of violence $\ldots \ldots \ldots \ldots \ldots \ldots \ldots \ldots \ldots \ldots \ldots$ & * & $\ldots$ & * & $\ldots$ \\
\hline 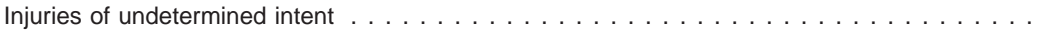 & 295 & 37 & 0.7 & 0.1 \\
\hline 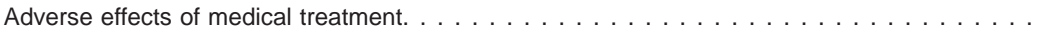 & 1,695 & 128 & 4.2 & 0.3 \\
\hline 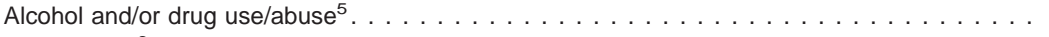 & 1,771 & 126 & 4.4 & 0.3 \\
\hline 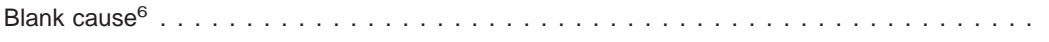 & 6,301 & 348 & 15.7 & 0.7 \\
\hline
\end{tabular}

. Category not applicable.

* Figure does not meet standard of reliability or precision.

0.0 Quantity more than zero but less than 0.05 .

${ }^{1}$ Based on the "Supplementary Classification of External Causes of Injury and Poisoning," International Classification of Diseases, 9th Revision, Clinical Modification (ICD-9-CM) (17) A detailed description of the ICD-9-CM E-codes used to create the groupings in this table is provided in the "Technical Notes."

2Includes drowning, firearms, and other mechanism.

${ }^{3}$ Includes assaults by firearms and explosives, and other mechanism.

${ }^{4}$ Includes injury by cutting and piercing instrument, and other and unspecified mechanism.

${ }^{5}$ Alcohol and drug abuse are not contained in the "Supplementary Classification of External Causes of Injury and Poisoning," but are frequently recorded as a cause of injury or poisoning.

${ }^{6}$ Includes illegible entries and blanks. 
Table 14. Number and percent of injury-related emergency department visits with corresponding standard errors, by body site of primary diagnosis: United States, 2003

\begin{tabular}{|c|c|c|c|c|}
\hline Body site ${ }^{1}$ & $\begin{array}{l}\text { Number of } \\
\text { visits in } \\
\text { thousands }\end{array}$ & $\begin{array}{l}\text { Standard } \\
\text { error in } \\
\text { thousands }\end{array}$ & $\begin{array}{c}\text { Percent } \\
\text { distribution }\end{array}$ & $\begin{array}{l}\text { Standard } \\
\text { error of } \\
\text { percent }\end{array}$ \\
\hline 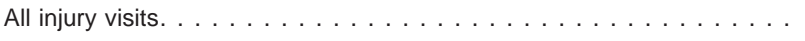 & 40,194 & 1,534 & 100.0 & $\cdots$ \\
\hline 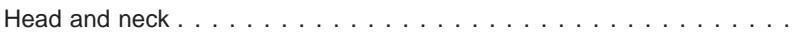 & 5,585 & 290 & 13.9 & 0.4 \\
\hline 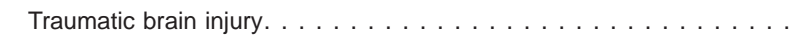 & 323 & 45 & 0.8 & 0.1 \\
\hline 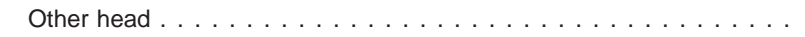 & 1,269 & 88 & 3.2 & 0.2 \\
\hline 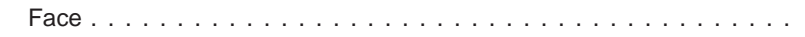 & 1,846 & 115 & 4.6 & 0.2 \\
\hline 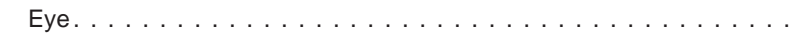 & 754 & 76 & 1.9 & 0.2 \\
\hline Head, face, and neck unspecified $\ldots \ldots \ldots \ldots \ldots$ & 1,393 & 117 & 3.5 & 0.2 \\
\hline 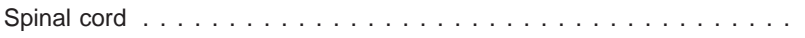 & * & * & & \\
\hline Vertebral column . . . . . . . . . . . . . . . . . . . & 1,923 & 126 & 4.8 & 0.3 \\
\hline 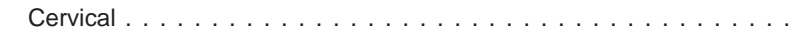 & 1,165 & 97 & 2.9 & 0.2 \\
\hline Thoracic and dorsal. . . . . . . . . . . . . . . . . . . & 161 & 26 & 0.4 & 0.1 \\
\hline Lumbar . . . . . . . . . . . . . . . . . . . . . . . & 575 & 61 & 1.4 & 0.1 \\
\hline 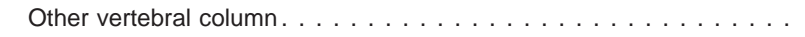 & * & $\ldots$ & * & $\ldots$ \\
\hline Torso . . . . . . . . . . . . . . . . . . . . . . . . . . . & 1,855 & 117 & 4.6 & 0.3 \\
\hline 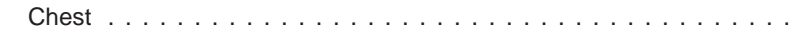 & 727 & 62 & 1.8 & 0.1 \\
\hline Abdomen . . . . . . . . . . . . . . . . . . . & 191 & 39 & 0.5 & 0.1 \\
\hline Pelvis and urogenital $\ldots \ldots \ldots \ldots \ldots \ldots \ldots \ldots \ldots \ldots$ & 325 & 44 & 0.8 & 0.1 \\
\hline Trunk. . . . . . . . . . . . . . . . . . . . . . . . . & 149 & 28 & 0.4 & 0.1 \\
\hline Back and buttocks. . . . . . . . . . . . . . . . . & 464 & 48 & 1.2 & 0.1 \\
\hline Upper extremity. . . . . . . . . . . . . . . . . . . . . . . & 7,847 & 355 & 19.5 & 0.4 \\
\hline Shoulder and upper arm . . . . . . . . . . . . . . . . . . . & 1,364 & 93 & 3.4 & 0.2 \\
\hline Forearm and elbow $\ldots \ldots \ldots \ldots \ldots \ldots \ldots \ldots \ldots \ldots$ & 1,383 & 88 & 3.4 & 0.2 \\
\hline Wrist, hand, and fingers $\ldots \ldots \ldots \ldots \ldots$ & 4,686 & 239 & 11.7 & 0.3 \\
\hline Other and unspecified upper extremity $\ldots \ldots \ldots \ldots \ldots \ldots$ & 414 & 51 & 1.0 & 0.1 \\
\hline Lower extremity. . . . . . . . . . . . . . . . . . . . . . . . . . . & 6,169 & 322 & 15.3 & 0.5 \\
\hline Нip . . . . . . . . . . . . . . . . . . & 525 & 87 & 1.3 & 0.2 \\
\hline Upper leg and thigh. . . . . . . . . . . . . . . . . & 111 & 28 & 0.3 & 0.1 \\
\hline Knee . . . . . . . . . . . . . . . . . . . . . . . . . & 481 & 52 & 1.2 & 0.1 \\
\hline Lower leg and ankle . . . . . . . . . . . . . . . . . . & 1,884 & 112 & 4.7 & 0.2 \\
\hline 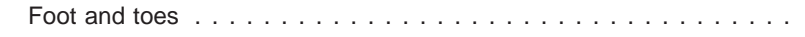 & 1,419 & 110 & 3.5 & 0.2 \\
\hline Other and unspecified lower extremity $\ldots \ldots \ldots \ldots \ldots$ & 1,748 & 145 & 4.3 & 0.3 \\
\hline System-wide . . . . . . . . . . . . . . . . . . . . . . & 1,836 & 122 & 4.6 & 0.2 \\
\hline Other and unspecified body site injuries. . . . . . . . . . . . . . & 2,629 & 180 & 6.5 & 0.4 \\
\hline Adverse effects and medical complications . . . . . . . . . . . . . & 1,331 & 90 & 3.3 & 0.2 \\
\hline 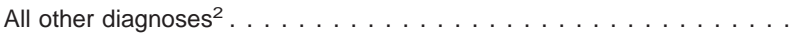 & 10,326 & 429 & 25.7 & 0.6 \\
\hline 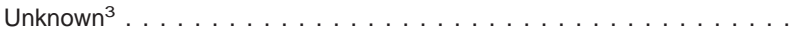 & 689 & 82 & 1.7 & 0.2 \\
\hline
\end{tabular}

. Category not applicable.

* Figure does not meet standard of reliability or precision.

${ }^{1}$ Based on the International Classification of Diseases, 9th Revision, Clinical Modication (ICD-9-CM) (17). A detailed description of the Barell Injury Diagnosis Matrix: Classification by Region of

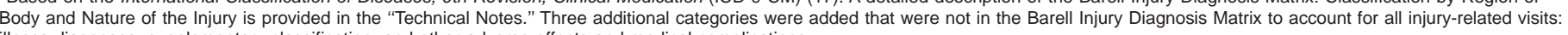
illness diagnoses, supplementary classification, and other adverse effects and medical complications.

${ }^{2}$ All other diagnoses include musculoskeletal system (710-739), symptoms and ill-defined conditions (780-799), skin and subcutaneous tissue (680-709), mental disorders (290-319), nervous system and sense organs (320-389), other illnesses (001-289, 390-677, 740-779), and supplementary classification (V01-V82).

${ }^{3}$ Includes blank diagnoses, uncodable diagnoses, and illegible diagnoses. 
Table 15. Number and percent of emergency department visits with corresponding standard errors, by diagnostic and screening services ordered or provided: United States, 2003

\begin{tabular}{|c|c|c|c|c|}
\hline Diagnostic and screening services ordered or provided & $\begin{array}{l}\text { Number of } \\
\text { visits in } \\
\text { thousands }^{1}\end{array}$ & $\begin{array}{l}\text { Standard } \\
\text { error in } \\
\text { thousands }\end{array}$ & $\begin{array}{l}\text { Percent } \\
\text { of visits }\end{array}$ & $\begin{array}{c}\text { Standard } \\
\text { error of } \\
\text { percent }\end{array}$ \\
\hline 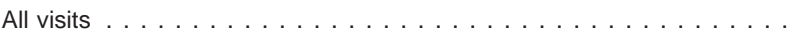 & 113,903 & 4,094 & $\ldots$ & $\ldots$ \\
\hline One or more diagnostic or screening services listed $\ldots \ldots \ldots \ldots$ & 102,185 & 3,870 & 89.7 & 0.9 \\
\hline None . . . . . . . . . . . . . . . . . . . . . . . . . & 9,933 & 938 & 8.7 & 0.8 \\
\hline Blank . . . . . . . . . . . . . . . . . . . & 1,786 & 422 & 1.6 & 0.4 \\
\hline \multicolumn{5}{|l|}{ Examinations and tests } \\
\hline 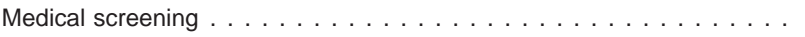 & 77,978 & 3,982 & 68.5 & 2.4 \\
\hline 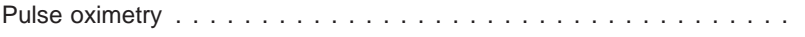 & 26,675 & 2,233 & 23.4 & 1.7 \\
\hline 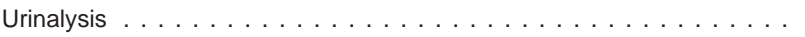 & 20,173 & 1,073 & 17.7 & 0.7 \\
\hline 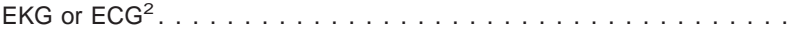 & 18,570 & 848 & 16.3 & 0.5 \\
\hline Mental status exam $\ldots \ldots \ldots \ldots \ldots \ldots \ldots \ldots \ldots \ldots \ldots \ldots$ & 11,009 & 1,489 & 9.7 & 1.3 \\
\hline Cardiac monitor. . . . . . . . . . . . . . . . . . . . . . . & 8,479 & 627 & 7.4 & 0.5 \\
\hline 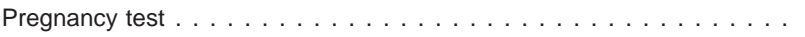 & 4,031 & 298 & 3.5 & 0.2 \\
\hline 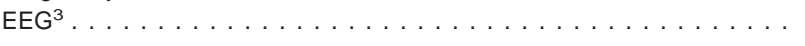 & 591 & 108 & 0.5 & 0.1 \\
\hline \multicolumn{5}{|l|}{ Imaging } \\
\hline 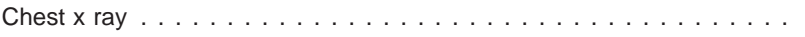 & 21,967 & 971 & 19.3 & 0.5 \\
\hline 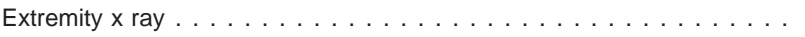 & 11,473 & 566 & 10.1 & 0.3 \\
\hline 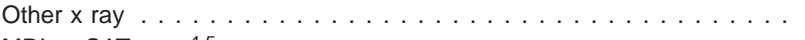 & 11,114 & 566 & 9.8 & 0.3 \\
\hline 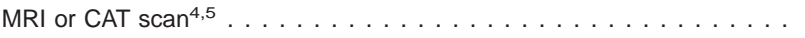 & 8,953 & 426 & 7.9 & 0.3 \\
\hline Ultrasound . . . . . . . . . . . . . . . . . . . . . . . . & 2,804 & 225 & 2.5 & 0.2 \\
\hline Other imaging . . . . . . . . . . . . . . . . . . . . & 2,470 & 233 & 2.2 & 0.2 \\
\hline 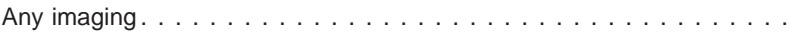 & 48,831 & 1,937 & 42.9 & 0.7 \\
\hline \multicolumn{5}{|l|}{ Blood tests } \\
\hline 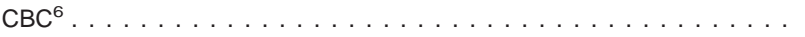 & 37,554 & 1,602 & 33.0 & 0.7 \\
\hline 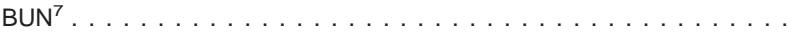 & 18,835 & 1,400 & 16.5 & 1.1 \\
\hline 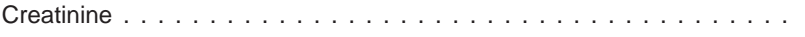 & 17,783 & 1,368 & 15.6 & 1.0 \\
\hline Glucose . . . . . . . . . . . . . . . . . . . . . . . . & 17,607 & 1,290 & 15.5 & 1.0 \\
\hline Electrolytes . . . . . . . . . . . . . . . . . . . . & 14,940 & 1,194 & 13.1 & 0.9 \\
\hline Lipids or cholesterol . . . . . . . . . . . . . . . . . . . . . . . . . . & 4,956 & 641 & 4.4 & 0.5 \\
\hline 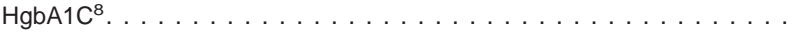 & 2,386 & 452 & 2.1 & 0.4 \\
\hline 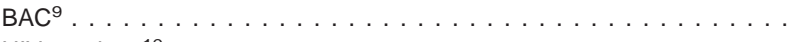 & 1,768 & 166 & 1.6 & 0.1 \\
\hline 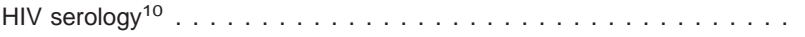 & 239 & 37 & 0.2 & 0.0 \\
\hline 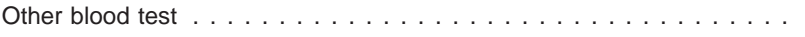 & 21,847 & 1,127 & 19.2 & 0.7 \\
\hline Any blood test listed $\ldots \ldots \ldots \ldots \ldots \ldots \ldots \ldots$ & 41,272 & 1,675 & 36.2 & 0.7 \\
\hline \multicolumn{5}{|l|}{ Cultures } \\
\hline Urine . . . . . . . . . . . . . . . . . . . . . . . & 5,198 & 376 & 4.6 & 0.3 \\
\hline Blood . . . . . . . . . . . . . . . . . . . . . & 3,642 & 320 & 3.2 & 0.2 \\
\hline Throat or rapid strep test $\ldots \ldots \ldots \ldots \ldots \ldots \ldots$ & 2,376 & 189 & 2.1 & 0.1 \\
\hline Cervical or urethral. . . . . . . . . . . . . . . . . . . . & 915 & 131 & 0.8 & 0.1 \\
\hline 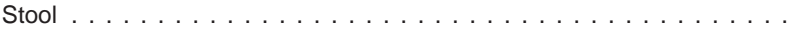 & 607 & 84 & 0.5 & 0.1 \\
\hline 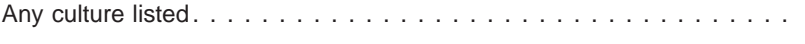 & 11,063 & 609 & 9.7 & 0.4 \\
\hline Other . . . . . . . . . . . . . . . . . . . . . . . . & 4,248 & 377 & 3.7 & 0.3 \\
\hline
\end{tabular}

Category not applicable.

0.0 Quantity more than zero but less than 0.05

${ }^{1}$ Total exceeds "All visits" because more than one service may be reported per visit.

${ }^{2} E K G$ or ECG is electrocardiogram.

${ }^{3} \mathrm{EEG}$ is electroencephalogram.

${ }^{4} \mathrm{MRI}$ is magnetic resonance imaging.

${ }^{5} \mathrm{CAT}$ is computerized axial tomography.

${ }^{6} \mathrm{CBC}$ is complete blood count.

${ }^{7} \mathrm{BUN}$ is blood urea nitrogen.

${ }^{8} \mathrm{HgbA1C}$ is glycohemoglobin.

${ }^{9} \mathrm{BAC}$ is blood alcohol concentration.

${ }^{10} \mathrm{HIV}$ is human immunodeficiency virus. 
Table 16. Number and percent of emergency department visits with corresponding standard errors, by selected procedures: United States, 2003

\begin{tabular}{|c|c|c|c|c|}
\hline Procedures provided & $\begin{array}{l}\text { Number of } \\
\text { visits in } \\
\text { thousands }^{1}\end{array}$ & $\begin{array}{l}\text { Standard } \\
\text { error in } \\
\text { thousands }\end{array}$ & $\begin{array}{l}\text { Percent } \\
\text { of visits }\end{array}$ & $\begin{array}{l}\text { Standard } \\
\text { error of } \\
\text { percent }\end{array}$ \\
\hline 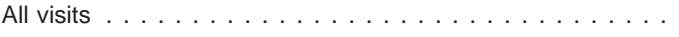 & 113,903 & 4,094 & $\cdots$ & $\cdots$ \\
\hline One or more procedures listed $\ldots \ldots \ldots \ldots$ & 51,691 & 2,310 & 45.4 & 1.2 \\
\hline None . . . . . . . . . . . . . . . . . . . . & 54,600 & 2,505 & 47.9 & 1.2 \\
\hline Blank . . . . . . . . . . . . . . . . . . . . & 7,612 & 1,088 & 6.7 & 1.0 \\
\hline IV fluids $^{2} \ldots \ldots \ldots \ldots \ldots \ldots \ldots$ & 24,886 & 1,279 & 21.8 & 0.8 \\
\hline Wound care $\ldots \ldots \ldots \ldots \ldots \ldots \ldots \ldots$ & 11,146 & 532 & 9.8 & 0.3 \\
\hline Orthopedic care $\ldots \ldots \ldots \ldots \ldots \ldots \ldots$ & 6,182 & 395 & 5.4 & 0.3 \\
\hline Eye or ENT care ${ }^{3} \ldots \ldots \ldots \ldots \ldots \ldots \ldots \ldots$ & 3,747 & 628 & 3.3 & 0.5 \\
\hline Bladder catheter . . . . . . . . . . . . . . . & 3,162 & 250 & 2.8 & 0.2 \\
\hline OB/GYN care $\ldots \ldots \ldots \ldots \ldots \ldots \ldots \ldots \ldots$ & 1,617 & 136 & 1.4 & 0.1 \\
\hline NG tube or gastric lavage ${ }^{5} \ldots \ldots \ldots \ldots \ldots$ & 470 & 50 & 0.4 & 0.0 \\
\hline Endotracheal intubation . . . . . . . . . . . . . & 379 & 48 & 0.3 & 0.0 \\
\hline Thrombolytic therapy . . . . . . . . . . . . . . . . . . . & *192 & 62 & *0.2 & 0.1 \\
\hline 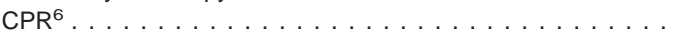 & 181 & 30 & 0.2 & 0.0 \\
\hline Other . . . . . . . . . . . . . . . . . . & 9,687 & 1,103 & 8.5 & 0.9 \\
\hline
\end{tabular}

Category not applicable.

0.0 Quantity more than zero but less than 0.05

${ }^{1}$ Total exceeds "All visits" because more than one procedure may be reported per visit.

${ }^{2}$ IV is intravenous.

${ }^{3} \mathrm{ENT}$ is ear, nose, and throat.

${ }^{4} \mathrm{OB} / \mathrm{GYN}$ is obstetrics/gynecology.

${ }^{5} \mathrm{NG}$ is nasogastric.

${ }^{6} \mathrm{CPR}$ is cardiopulmonary resuscitation.

Table 17. Number and percent distribution of emergency department visits with corresponding standard errors, by medication therapy and number of medications provided or prescribed: United States, 2003

\begin{tabular}{|c|c|c|c|c|}
\hline Medication therapy ${ }^{1}$ & $\begin{array}{l}\text { Number of } \\
\text { visits in } \\
\text { thousands }\end{array}$ & $\begin{array}{l}\text { Standard } \\
\text { error in } \\
\text { thousands }\end{array}$ & $\begin{array}{c}\text { Percent } \\
\text { distribution }\end{array}$ & $\begin{array}{c}\text { Standard } \\
\text { error of } \\
\text { percent }\end{array}$ \\
\hline 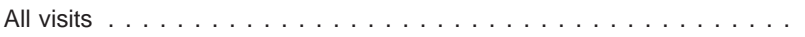 & 113,903 & 4,094 & 100.0 & $\ldots$ \\
\hline 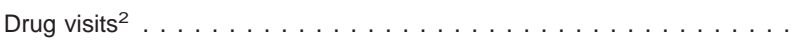 & 87,999 & 3,398 & 77.3 & 0.8 \\
\hline Visits without mention of medication $\ldots \ldots \ldots \ldots \ldots$ & 25,904 & 1,169 & 22.7 & 0.8 \\
\hline \multicolumn{5}{|l|}{ Number of medications provided or prescribed } \\
\hline 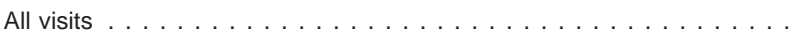 & 113,903 & 4,094 & 100.0 & $\ldots$ \\
\hline 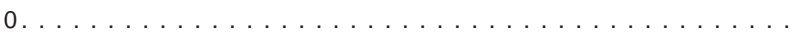 & 25,904 & 1,169 & 22.7 & 0.8 \\
\hline 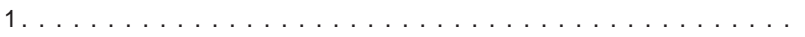 & 30,436 & 1,216 & 26.7 & 0.6 \\
\hline 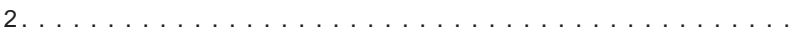 & 26,072 & 1,099 & 22.9 & 0.4 \\
\hline 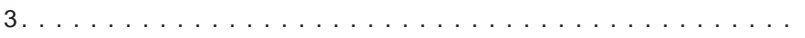 & 15,268 & 756 & 13.4 & 0.4 \\
\hline 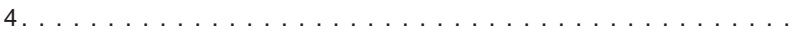 & 7,499 & 424 & 6.6 & 0.3 \\
\hline 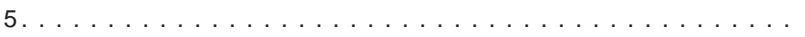 & 3,715 & 269 & 3.3 & 0.2 \\
\hline 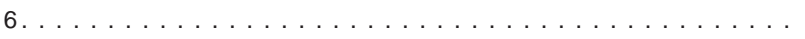 & 1,861 & 157 & 1.6 & 0.1 \\
\hline 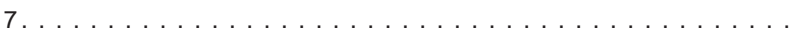 & 1,149 & 140 & 1.0 & 0.1 \\
\hline 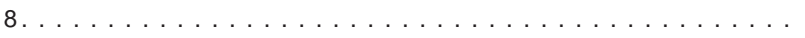 & 1,999 & 311 & 1.8 & 0.3 \\
\hline
\end{tabular}

Category not applicable.

0.0 Quantity more than zero but less than 0.05 .

Includes prescription drugs, over-the-counter preparations, immunizations, and desensitizing agents.

${ }^{2}$ Visits at which one or more drugs were provided or prescribed.

NOTE: Numbers may not add to totals because of rounding. 
Table 18. Number and percent of drug mentions for the $\mathbf{2 0}$ most frequently occurring therapeutic drug classes at emergency department visits with corresponding standard errors: United States, 2003

\begin{tabular}{|c|c|c|c|c|}
\hline Therapeutic class ${ }^{1}$ & $\begin{array}{c}\text { Number of } \\
\text { occurrences } \\
\text { in thousands }\end{array}$ & $\begin{array}{c}\text { Standard } \\
\text { error in } \\
\text { thousands }\end{array}$ & $\begin{array}{l}\text { Percent } \\
\text { of drug } \\
\text { mentions }^{2}\end{array}$ & $\begin{array}{c}\text { Standard } \\
\text { error of } \\
\text { percent }\end{array}$ \\
\hline Narcotic analgesics . . . . . . . . . . . . . . . & 29,854 & 1,401 & 14.1 & 0.7 \\
\hline 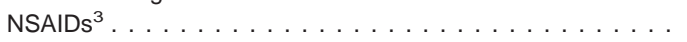 & 25,615 & 1,274 & 12.1 & 0.6 \\
\hline Nonnarcotic analgesics . . . . . . . . . . . . . . . . . . & 15,682 & 871 & 7.4 & 0.5 \\
\hline 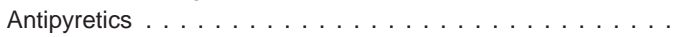 & 14,856 & 844 & 7.0 & 0.5 \\
\hline 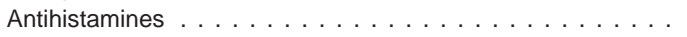 & 14,320 & 719 & 6.7 & 0.4 \\
\hline Vertigo, motion sickness, vomiting . . . . . . . . . . . & 11,745 & 599 & 5.5 & 0.4 \\
\hline Sedatives and hypnotics . . . . . . . . . . . . . . & 10,679 & 553 & 5.0 & 0.3 \\
\hline Antiasthmatics, bronchodilators . . . . . . . . . . . & 9,271 & 571 & 4.4 & 0.4 \\
\hline Cephalosporins . . . . . . . . . . . . . . . . . & 8,670 & 526 & 4.1 & 0.3 \\
\hline 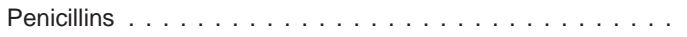 & 7,453 & 451 & 3.5 & 0.3 \\
\hline Acid or peptic disorders . . . . . . . . . . . . . . . & 6,764 & 449 & 3.2 & 0.3 \\
\hline Replenishers, regulators of electrolytes, water balance . . . . & 5,455 & 461 & 2.6 & 0.4 \\
\hline Adrenal corticosteroids $\ldots \ldots \ldots \ldots \ldots \ldots$ & 5,306 & 297 & 2.5 & 0.2 \\
\hline Erythromycins, lincosamides, macrolides . . . . . . . . . . & 5,169 & 330 & 2.4 & 0.2 \\
\hline Antianxiety agents $\ldots \ldots \ldots \ldots \ldots \ldots \ldots$ & 4,364 & 241 & 2.1 & 0.2 \\
\hline Antiarthritics . . . . . . . . . . . . . . . . & 4,323 & 315 & 2.0 & 0.2 \\
\hline Quinolones . . . . . . . . . . . . . . . . . . . . . . & 4,118 & 243 & 1.9 & 0.2 \\
\hline Skeletal muscle hyperactivity $\ldots \ldots \ldots \ldots$ & 4,029 & 241 & 1.9 & 0.2 \\
\hline Vaccines or antisera $\ldots \ldots \ldots \ldots \ldots \ldots$ & 3,289 & 247 & 1.6 & 0.2 \\
\hline 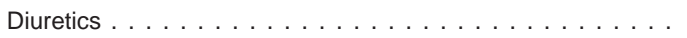 & 3,089 & 280 & 1.5 & 0.2 \\
\hline
\end{tabular}

${ }^{1}$ Based on the standard four-digit drug classification used in the National Drug Code Directory, 1995 edition (20).

${ }^{2}$ Based on an estimated $212,156,000$ drug mentions at emergency department visits in 2003. Total of all therapeutic classes will exceed total drug mentions because up to three classes may be coded for each drug.

${ }^{3}$ NSAIDs are nonsteroidal anti-inflammatory drugs.

Table 19. Number and rate of generic substances for the $\mathbf{2 0}$ most frequently occurring generic substances in drug mentions at emergency department visits with corresponding standard errors: United States, 2003

\begin{tabular}{|c|c|c|c|c|}
\hline Generic substance & $\begin{array}{l}\text { Number of } \\
\text { occurrences } \\
\text { in thousands }{ }^{1}\end{array}$ & $\begin{array}{l}\text { Standard } \\
\text { error in } \\
\text { thousands }\end{array}$ & $\begin{array}{c}\text { Number } \\
\text { of generic } \\
\text { substances } \\
\text { per } 100 \text { drug } \\
\text { mentions }{ }^{2}\end{array}$ & $\begin{array}{c}\text { Standard } \\
\text { error } \\
\text { of rate }\end{array}$ \\
\hline Acetaminophen . . . . . . . . . . . . . . . . . . . . & 30,080 & 1,372 & 14.2 & 0.6 \\
\hline Ibuprofen. . . . . . . . . . . . . . . . . . . . . & 15,611 & 902 & 7.4 & 0.5 \\
\hline Hydrocodone . . . . . . . . . . . . . . . . . . & 11,332 & 664 & 5.3 & 0.4 \\
\hline 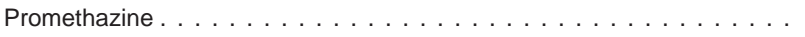 & 9,043 & 502 & 4.3 & 0.3 \\
\hline 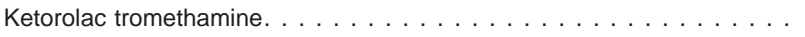 & 6,207 & 356 & 2.9 & 0.2 \\
\hline Albuterol . . . . . . . . . . . . . . . . . . . . & 5,753 & 363 & 2.7 & 0.2 \\
\hline Amoxicillin . . . . . . . . . . . . . . . . . . & 5,627 & 395 & 2.7 & 0.3 \\
\hline Morphine. . . . . . . . . . . . . . . . . . . . . . & 4,290 & 293 & 2.0 & 0.2 \\
\hline Meperidine. . . . . . . . . . . . . . . . . . . . . . . . & 3,681 & 295 & 1.7 & 0.2 \\
\hline 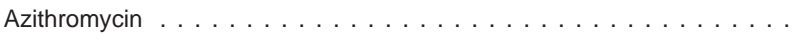 & 3,678 & 251 & 1.7 & 0.2 \\
\hline 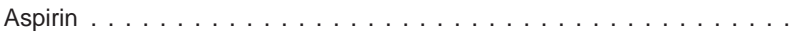 & 3,571 & 281 & 1.7 & 0.2 \\
\hline 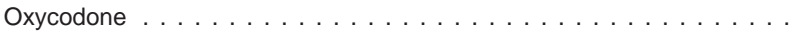 & 3,539 & 317 & 1.7 & 0.3 \\
\hline 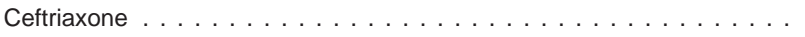 & 3,333 & 290 & 1.6 & 0.2 \\
\hline Cephalexin. . . . . . . . . . . . . . . . . . . . . . & 3,310 & 222 & 1.6 & 0.2 \\
\hline 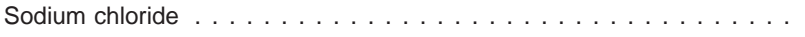 & 3,293 & 418 & 1.6 & 0.4 \\
\hline Diphenhydramine. . . . . . . . . . . . . . . . . . . . . . & 2,980 & 188 & 1.4 & 0.1 \\
\hline Tetanus toxoid $\ldots \ldots \ldots \ldots \ldots \ldots \ldots \ldots \ldots \ldots$ & 2,924 & 238 & 1.4 & 0.2 \\
\hline Nitroglycerin . . . . . . . . . . . . . . . . . . . . . & 2,772 & 254 & 1.3 & 0.2 \\
\hline Lorazepam. . . . . . . . . . . . . . . . . . . . . . . . . . . . . . . . & 2,479 & 155 & 1.2 & 0.1 \\
\hline 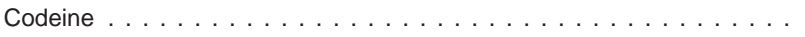 & 2,465 & 188 & 1.2 & 0.1 \\
\hline
\end{tabular}

${ }^{1}$ Frequency of mention combines single-ingredient agents with mentions of the agent as an ingredient in a combination drug. 
Table 20. Number, percent distribution, and therapeutic classification for the $\mathbf{2 0}$ drugs most frequently prescribed at emergency department visits with corresponding standard errors, by entry name of drug: United States, 2003

\begin{tabular}{|c|c|c|c|c|c|}
\hline Entry name of drug ${ }^{1}$ & $\begin{array}{l}\text { Number of } \\
\text { drug mentions } \\
\text { in thousands }\end{array}$ & $\begin{array}{l}\text { Standard } \\
\text { error in } \\
\text { thousands }\end{array}$ & $\begin{array}{l}\text { Percent } \\
\text { distribution }\end{array}$ & $\begin{array}{l}\text { Standard } \\
\text { error of } \\
\text { percent }\end{array}$ & Therapeutic class ${ }^{2}$ \\
\hline All drug mentions. . . . . . . . . . . . & 212,155 & 9,648 & 100.0 & $\cdots$ & $\cdots$ \\
\hline Tylenol . . . . . . . . . . . . . . . . . & 11,096 & 675 & 5.2 & 0.2 & Nonnarcotic analgesics; antipyretics \\
\hline Motrin. . . . . . . . . . . . . & 9,839 & 691 & 4.6 & 0.2 & NSAIDs $^{3}$ \\
\hline Phenergan. . . . . . . . . . . & 8,558 & 496 & 4.0 & 0.2 & $\begin{array}{l}\text { Sedatives and hypnotics; vertigo, motion sickness, vomiting; } \\
\text { antihistamines }\end{array}$ \\
\hline Vicodin . . . . . . . . . . . . . . & 6,896 & 561 & 3.3 & 0.2 & Narcotic analgesics \\
\hline Toradol . . . . . . . . . . . . . . . . . . . & 6,062 & 349 & 2.9 & 0.1 & $\mathrm{NSAIDs}^{3}$ \\
\hline Albuterol . . . . . . . . . . . . & 4,748 & 317 & 2.2 & 0.1 & Antiasthmatics, bronchodilators \\
\hline Ibuprofen. . . . . . . . . . . . & 4,299 & 326 & 2.0 & 0.1 & NSAIDs $^{3}$ \\
\hline Demerol . . . . . . . . . . . . . & 3,608 & 298 & 1.7 & 0.1 & Narcotic analgesics \\
\hline Lortab. . . . . . . . . . . . . . . . . . & 3,045 & 346 & 1.4 & 0.2 & Narcotic analgesics \\
\hline Rocephin. . . . . . . . . . . . . . & 3,023 & 282 & 1.4 & 0.1 & Cephalosporins \\
\hline Keflex. . . . . . . . . . . . & 2,979 & 212 & 1.4 & 0.1 & Cephalosporins \\
\hline Morphine. . . . . . . . . . . . . . & 2,979 & 236 & 1.4 & 0.1 & Narcotic analgesics \\
\hline Percocet-5. . . . . . . . . . & 2,978 & 285 & 1.4 & 0.1 & Narcotic analgesics \\
\hline Benadryl . . . . . . . . . . . . . . . & 2,824 & 171 & 1.3 & 0.1 & Antihistamines \\
\hline Normal saline . . . . . . . . . . . . & 2,750 & 380 & 1.3 & 0.2 & $\begin{array}{l}\text { Replenishers, regulators of electrolytes, water } \\
\text { balance; pharmaceutical aids; surgical aids }\end{array}$ \\
\hline Zithromax . . . . . . . . . . . & 2,659 & 192 & 1.3 & 0.1 & Erythromycins, lincosamides, macrolides \\
\hline Amoxicillin . . . . . . . . . . . . & 2,566 & 189 & 1.2 & 0.1 & Penicillins \\
\hline Levaquin . . . . . . . . . . . . . . . . . & 2,394 & 177 & 1.1 & 0.1 & Quinolones \\
\hline Prednisone . . . . . . . . . . & 2,393 & 153 & 1.1 & 0.1 & Adrenal corticosteroids \\
\hline Ativan. . . . . . . . . . . . . . . & 2,324 & 146 & 1.1 & 0.1 & Antianxiety agents \\
\hline All other mentions $\ldots \ldots \ldots \ldots$ & 124,133 & 6,385 & 58.5 & 0.8 & $\ldots$ \\
\hline
\end{tabular}

.. Category not applicable.

${ }^{1}$ The entry made by the hospital staff on the prescription or other medical records. This may be a trade name, generic name, or desired therapeutic effect.

${ }^{2}$ Therapeutic classification is based on the National Drug Code Directory, 1995 edition (20). In cases where a drug had more than one therapeutic use, it was classified under each therapeutic class.

${ }^{3} \mathrm{NSAIDs}$ are nonsteroidal anti-inflammatory drugs.

NOTE: Numbers may not add to totals because of rounding.

Table 21. Number and percent of emergency department visits with corresponding standard errors, by providers seen: United States, 2003

\begin{tabular}{|c|c|c|c|c|}
\hline Type of provider & $\begin{array}{l}\text { Number of } \\
\text { visits in } \\
\text { thousands }\end{array}$ & $\begin{array}{l}\text { Standard } \\
\text { error in } \\
\text { thousands }\end{array}$ & $\begin{array}{l}\text { Percent } \\
\text { of visits }\end{array}$ & $\begin{array}{c}\text { Standard } \\
\text { error of } \\
\text { percent }\end{array}$ \\
\hline … & 113,903 & 4,094 & $\ldots$ & .. \\
\hline Any physician $\ldots \ldots \ldots \ldots \ldots \ldots \ldots \ldots$ & 105,219 & 3,815 & 92.4 & 0.8 \\
\hline Staff physician $\ldots \ldots \ldots \ldots \ldots \ldots \ldots \ldots \ldots$ & 100,017 & 3,810 & 87.8 & 1.3 \\
\hline Other physician $\ldots \ldots \ldots \ldots \ldots \ldots \ldots$ & 9,929 & 1,210 & 8.7 & 1.0 \\
\hline Resident or intern . . . . . . . . . . . . . . . . . . . . & 9,571 & 1,296 & 8.4 & 1.1 \\
\hline 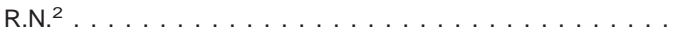 & 103,138 & 4,152 & 90.5 & 1.4 \\
\hline Other technician $\ldots \ldots \ldots \ldots \ldots \ldots \ldots \ldots \ldots \ldots$ & 32,108 & 2,227 & 28.2 & 1.7 \\
\hline E.M.T. ${ }^{3} \ldots \ldots \ldots \ldots \ldots \ldots \ldots \ldots \ldots$ & 9,881 & 1,351 & 8.7 & 1.1 \\
\hline Physician assistant. . . . . . . . . . . . . . . & 7,459 & 1,077 & 6.5 & 0.9 \\
\hline L.P.N. ${ }^{4} \ldots \ldots \ldots \ldots \ldots \ldots \ldots \ldots \ldots \ldots \ldots$ & 7,203 & 1,135 & 6.3 & 1.0 \\
\hline Nurse practitioner $\ldots \ldots \ldots \ldots \ldots \ldots \ldots \ldots$ & 2,047 & 319 & 1.8 & 0.3 \\
\hline Other $\ldots \ldots \ldots \ldots \ldots \ldots \ldots \ldots \ldots \ldots$ & 9,010 & 1,746 & 7.9 & 1.5 \\
\hline Blank . . . . . . & 1,047 & 132 & 0.9 & 0.1 \\
\hline
\end{tabular}

Category not applicable.

"Total exceeds "All visits" because more than one provider may be reported per visit.

${ }^{2}$ R.N. is registered nurse.

${ }^{3}$ E.M.T. is emergency medical technician.

${ }^{4}$ L.P.N. is licensed practical nurse. 
Table 22. Number and percent of emergency department visits with corresponding standard errors, by visit disposition: United States, 2003

\begin{tabular}{|c|c|c|c|c|}
\hline Disposition & $\begin{array}{l}\text { Number of } \\
\text { visits in } \\
\text { thousands }{ }^{1}\end{array}$ & $\begin{array}{l}\text { Standard } \\
\text { error in } \\
\text { thousands }\end{array}$ & $\begin{array}{l}\text { Percent } \\
\text { of visits }\end{array}$ & $\begin{array}{l}\text { Standard } \\
\text { error of } \\
\text { percent }\end{array}$ \\
\hline 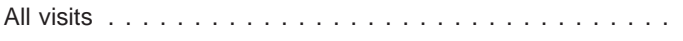 & 113,903 & 4,094 & $\cdots$ & $\cdots$ \\
\hline \multicolumn{5}{|l|}{ Admitted or transferred or died } \\
\hline Admit to hospital ${ }^{2} \ldots \ldots \ldots \ldots \ldots \ldots \ldots$ & 15,809 & 844 & 13.9 & 0.5 \\
\hline Transfer to other facility . . . . . . . . . . . . . . . . & 2,219 & 148 & 1.9 & 0.1 \\
\hline Admit to ICU or $\mathrm{CCU}^{3} \ldots \ldots \ldots \ldots \ldots \ldots$ & 1,511 & 146 & 1.3 & 0.1 \\
\hline Admit to ED for observation $\ldots \ldots \ldots \ldots \ldots$ & 384 & 65 & 0.3 & 0.1 \\
\hline $\mathrm{DOA} / \mathrm{died}$ in $\mathrm{ED}^{4,5} \ldots \ldots \ldots \ldots \ldots \ldots \ldots$ & 317 & 42 & 0.3 & 0.0 \\
\hline \multicolumn{5}{|l|}{ Referred or returned to physician } \\
\hline Refer to other physician or clinic for $\mathrm{FU}^{6} \ldots \ldots \ldots \ldots$ & 53,560 & 2,827 & 47.0 & 1.6 \\
\hline Return to referring physician $\ldots \ldots \ldots \ldots \ldots$ & 15,838 & 1,378 & 13.9 & 1.2 \\
\hline \multicolumn{5}{|l|}{ Left or referred out from triage } \\
\hline Left before being seen $\ldots \ldots \ldots \ldots \ldots \ldots$ & 1,918 & 176 & 1.7 & 0.1 \\
\hline 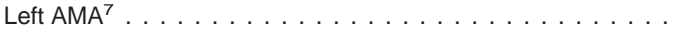 & 1,175 & 103 & 1.0 & 0.1 \\
\hline Refer out from triage without treatment $\ldots \ldots \ldots \ldots$ & ${ }^{*} 188$ & 68 & ${ }^{*} 0.2$ & 0.1 \\
\hline \multicolumn{5}{|l|}{ Returned or referred to other treatment } \\
\hline Return to nonphysician treatment or support service . . . . . . & 601 & 73 & 0.5 & 0.1 \\
\hline Refer to alcohol or drug treatment program. . . . . . . . . & 460 & 54 & 0.4 & 0.0 \\
\hline \multicolumn{5}{|l|}{ Other } \\
\hline Return if needed, PRN or appointment ${ }^{8} \ldots \ldots \ldots \ldots$ & 46,991 & 3,031 & 41.3 & 2.1 \\
\hline No followup planned. . . . . . . . . . . . . . . . . . . & 7,145 & 1,070 & 6.3 & 0.9 \\
\hline Other . . . . . . . . . . . . . . . . . . . . . & 849 & 127 & 0.7 & 0.1 \\
\hline 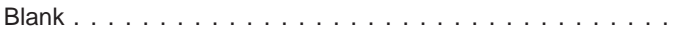 & 775 & 163 & 0.7 & 0.1 \\
\hline
\end{tabular}

\footnotetext{
.. Category not applicable.

${ }^{*}$ Figure does not meet standard of reliability or precision.

0.0 Quantity more than zero but less than 0.05 .

${ }^{1}$ Total exceeds "All visits" because more than one disposition may be reported per visit.

${ }^{2}$ Includes those admitted to ICU or CCU.

${ }^{3} \mathrm{ICU}$ or $\mathrm{CCU}$ is intensive care unit or critical care unit or coronary care unit and is a subset of those admitted to hospital.

${ }^{4} \mathrm{DOA}$ is dead on arrival.

${ }^{5} \mathrm{ED}$ is emergency department.

${ }^{6} \mathrm{FU}$ is followup.

${ }^{7} \mathrm{AMA}$ is against medical advice.

${ }^{8} \mathrm{PRN}$ is "as needed."
} 
Table 23. Percent distribution of emergency departments and corresponding standard errors by selected hospital or emergency department characteristics, according to size of annual visit volume: United States, 2003

\begin{tabular}{|c|c|c|c|c|c|c|c|c|}
\hline \multirow[b]{2}{*}{ Hospital or emergency department characteristic } & \multicolumn{2}{|c|}{ All 24-hour EDs } & \multicolumn{2}{|c|}{ Small (less than 20,000) } & \multicolumn{2}{|c|}{ Medium $(20,000-50,000)$} & \multicolumn{2}{|c|}{ Large (more than 50,000 ) } \\
\hline & $\begin{array}{c}\text { Percent } \\
\text { distribution }\end{array}$ & $\begin{array}{l}\text { Standard } \\
\text { error of } \\
\text { percent }\end{array}$ & $\begin{array}{c}\text { Percent } \\
\text { distribution }\end{array}$ & $\begin{array}{l}\text { Standard } \\
\text { error of } \\
\text { percent }\end{array}$ & $\begin{array}{c}\text { Percent } \\
\text { distribution }\end{array}$ & $\begin{array}{l}\text { Standard } \\
\text { error of } \\
\text { percent }\end{array}$ & $\begin{array}{l}\text { Percent } \\
\text { distribution }\end{array}$ & $\begin{array}{l}\text { Standard } \\
\text { error of } \\
\text { percent }\end{array}$ \\
\hline All EDs ${ }^{1} \ldots \ldots \ldots \ldots \ldots \ldots \ldots$ & 100.0 & $\ldots$ & 100.0 & $\ldots$ & 100.0 & $\ldots$ & 100.0 & $\ldots$ \\
\hline \multicolumn{9}{|l|}{ Geographic region } \\
\hline Northeast . . . . . . . . . . . . . . . . . . & 16.0 & 1.3 & *9.6 & 1.6 & 25.6 & 3.3 & 18.6 & 3.2 \\
\hline Midwest. . . . . . . . . . . . . . . . . . & 28.6 & 2.1 & 29.9 & 4.0 & 24.6 & 3.3 & 34.5 & 4.5 \\
\hline South . . . . . . . . . . . . . . . & 38.5 & 2.4 & 44.5 & 4.5 & 33.4 & 3.9 & 24.7 & 4.5 \\
\hline West. . . . . . . . . . . . . . . . . & 16.9 & 1.4 & 16.0 & 3.1 & 16.4 & 2.9 & 22.2 & 4.9 \\
\hline \multicolumn{9}{|l|}{ Metropolitan status } \\
\hline$\ldots \ldots \ldots \ldots \ldots \ldots$ & 57.7 & 3.2 & 30.5 & 5.1 & 87.8 & 3.5 & 100.0 & $\ldots$ \\
\hline Non-MSA ${ }^{2} \ldots \ldots \ldots \ldots \ldots \ldots$ & 42.3 & 3.2 & 69.5 & 5.1 & ${ }^{*} 12.2$ & 3.5 & ${ }^{*} 0.0$ & 0.0 \\
\hline \multicolumn{9}{|l|}{ Ownership } \\
\hline Voluntary . . . . . . . . . . . . . . . . . . & 61.9 & 4.2 & 51.6 & 7.3 & 72.3 & 4.1 & 80.6 & 3.6 \\
\hline Government . . . . . . . . . . . . . . . . . & 26.6 & 4.2 & 36.2 & 7.5 & 15.2 & 2.9 & 13.9 & 2.8 \\
\hline Proprietary . . . . . . . . . . . . . . . . . . . & 11.5 & 1.6 & 12.2 & 2.9 & 12.5 & 3.5 & *5.5 & 3.0 \\
\hline \multicolumn{9}{|l|}{ Type of hospital } \\
\hline General . . . . . . . . . . . . . . . . . . . . . . & 95.0 & 1.4 & 95.3 & 2.3 & 96.4 & 1.4 & 89.8 & 3.6 \\
\hline Specialty . . . . . . . . . . . . . . . . . . . . & 5.0 & 1.4 & *4.7 & 2.3 & *3.6 & 1.4 & ${ }^{*} 10.2$ & 3.6 \\
\hline \multicolumn{9}{|l|}{ Medical school affiliation } \\
\hline 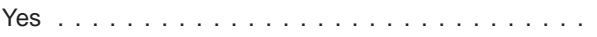 & 31.5 & 3.3 & *17.9 & 5.3 & 40.7 & 4.4 & 70.8 & 5.8 \\
\hline 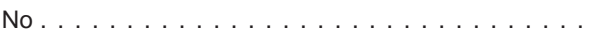 & 68.5 & 3.3 & 82.1 & 5.3 & 59.3 & 4.4 & 29.2 & 5.8 \\
\hline \multicolumn{9}{|l|}{$\begin{array}{l}\text { Outpatient department } \\
\text { with physician-supervised clinics }\end{array}$} \\
\hline 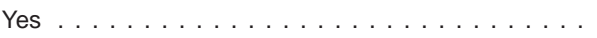 & 45.3 & 4.0 & 39.0 & 6.7 & 47.7 & 4.3 & 68.7 & 7.4 \\
\hline 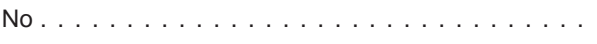 & 54.7 & 4.0 & 61.0 & 6.7 & 52.3 & 4.3 & 31.3 & 7.4 \\
\hline \multicolumn{9}{|l|}{ Number of emergency service areas } \\
\hline One ...................... & 95.9 & 0.9 & 100.0 & 0.0 & 96.5 & 1.3 & 74.0 & 6.8 \\
\hline Multiple . . . . . . . . . . . . . . . . . . & 4.1 & 0.9 & ${ }^{*} 0.0$ & 0.0 & *3.5 & 1.3 & 26.0 & 6.8 \\
\hline \multicolumn{9}{|l|}{ Number of levels in nursing triage system } \\
\hline Three . . . . . . . . . . . . . . . . . . . . . & 47.2 & 3.8 & 44.3 & 6.2 & 56.7 & 4.6 & 32.9 & 5.4 \\
\hline Four . . . . . . . . . . . . . . . . . & 19.6 & 3.3 & 23.5 & 5.4 & 11.5 & 2.6 & 24.8 & 4.8 \\
\hline 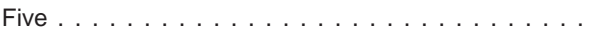 & 19.6 & 2.9 & *13.3 & 4.4 & 23.8 & 4.1 & 38.0 & 5.3 \\
\hline No triage $\ldots \ldots \ldots \ldots \ldots \ldots \ldots \ldots$ & *6.6 & 2.0 & *7.6 & 3.1 & *5.9 & 2.5 & *3.7 & 2.0 \\
\hline Unknown . . . . . . . . . . . . . . . . . . . & *7.0 & 2.1 & *11.3 & 3.7 & *2.1 & 1.2 & *0.6 & 0.6 \\
\hline \multicolumn{9}{|l|}{ Board-certified emergency medicine physician ${ }^{3}$} \\
\hline Yes $\ldots \ldots \ldots \ldots \ldots \ldots \ldots$ & 63.5 & 3.5 & 47.3 & 5.4 & 81.6 & 3.2 & 87.9 & 2.6 \\
\hline 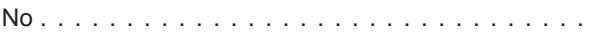 & 27.4 & 4.0 & 42.4 & 6.7 & *10.6 & 2.7 & *4.1 & 1.9 \\
\hline Unknown . . . . . . . . . . . . . . . . . . & 9.2 & 2.5 & *10.2 & 4.4 & *7.8 & 2.0 & *7.9 & 1.8 \\
\hline \multicolumn{9}{|l|}{$\begin{array}{c}\text { Board-certified pediatric } \\
\text { emergency medicine physician }{ }^{3}\end{array}$} \\
\hline 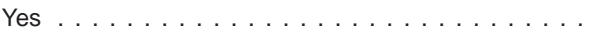 & 18.1 & 2.6 & *10.2 & 3.8 & 23.5 & 3.9 & 40.5 & 4.8 \\
\hline 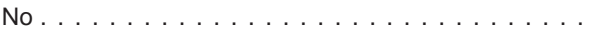 & 73.4 & 3.1 & 81.1 & 4.8 & 68.2 & 4.0 & 51.0 & 4.7 \\
\hline 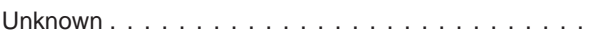 & 8.5 & 2.0 & *8.7 & 3.3 & *8.3 & 2.1 & *8.5 & 1.9 \\
\hline
\end{tabular}

\footnotetext{
.. Category not applicable.

* Figure does not meet standard of reliability or precision.

0.0 Quantity more than zero but less than 0.05 .

${ }^{1} E D$ is emergency department.

${ }^{2} \mathrm{MSA}$ is metropolitan statistical area.

${ }^{3}$ On duty or on-call 24 hours a day and 7 days a week.
} 


\section{Technical notes}

\section{Data collection}

The NHAMCS data collection is authorized under section 306 of the Public Health Service Act (Title 42, U.S. Code), S242k. Participation is voluntary. In 2003, a sample of 546 general and short-stay hospitals was selected from a sampling frame constructed from products of Verispan, L.L.C., specifically "Healthcare Market Index" and "Hospital Market Profiling Solution." These products were formerly known as the SMG Hospital Database.

A special supplement of 66 hospitals was added to the NHAMCS in 2003 in order to increase reliability of emergency department estimates for rural and proprietary hospitals. For the supplemental sample, a three-stage design was used where the first-stage was a selection of hospitals regardless of PSUs. The supplemental sample used a list-frame from the Verispan file of hospitals indicating the presence of an emergency department. Within sampled hospitals, selected ESAs within emergency departments and patient visits within ESAs were sampled. The design of the sample within hospitals for the additional sample was identical to the within-hospital sample for the four-stage design. Funding for the supplemental sample was given by the Office of the Assistant Secretary for Planning and Evaluation in the U.S. Department of Health and Human Services and the Bureau of Maternal and Child Health of the Health Resources and Services Administration.

Together, a sample of 546 hospitals was selected for the 2003 NHAMCS, of which 443 were in scope and had eligible EDs. Of the 443 in-scope hospitals with EDs, 406 participated, and 405 of them provided at least half of the PRFs expected based on the total number of visits seen, and hence were considered to be fully or adequately responding, for an unweighted ED response rate of 91.4 percent. A sample of 506 ESAs was selected from the EDs, and 475 of them provided 40,253 Patient Record forms (PRFs). Of these 506 ESAs, 472 of them responded fully or adequately, yielding an ESA sampling response rate of 93.3 percent, and an overall unweighted two-stage sampling response rate of 85.3 percent. In Advance Data reports prior to 2001, only the ED and ESA participation rates were provided (i.e., information on completed Patient Record forms was not factored into the response rates); these varied between 94 and 97 percent. Starting with the 2001 survey, the response rate calculation has taken into account both the ED and ESA participation rates.

The U.S. Census Bureau, acting as the data collection agent for the survey, provided training to field representatives (FRs) throughout the nation who, in turn, oversaw data collection at the hospital and clinic level. FRs contacted the sampled hospitals for induction into the survey after an advance letter was mailed by NCHS notifying the hospitals of their selection for the survey. Hospital staff was instructed to complete the information requested on the Patient Record forms (figure I). However, for 45.5 percent of the sampled records, FRs abstracted the data from medical records or computer printouts.

\section{Health Insurance Portability and Accountability Act}

In April 2003, the Privacy Rule of HIPAA was implemented to establish minimum Federal standards for safeguarding the privacy of individually identifiable health information. No personally identifying information, such as patient's name, address, or Social Security number, is collected in the NHAMCS. Data collection is authorized by Section 306 of the Public Health Service Act (Title 42, U.S. Code, 242k). All information collected is held in the strictest confidence according to law [Section 308(d) of the Public Health Service Act (42, U.S. Code, 242m(d))] and the Confidential Information Protection and Statistical Efficiency Act (Title 5 of PL 107-347). The NHAMCS protocol was approved by the NCHS Research Ethics Review Board in February 2003. Waivers of the requirements to obtain informed consent of patients and patient authorization for release of patient medical record data by health care providers were granted.
In the spring of 2003, the NHAMCS implemented additional data collection procedures to help providers ensure patient confidentiality. Census Bureau field representatives were trained on how the Privacy Rule allows hospitals to make disclosures of protected health information without patient authorization for public health purposes and for research that has been approved by a Research Ethics Review Board. Hospitals were encouraged to accept a data use agreement between themselves and CDC's NCHS because the Privacy Rule allows hospitals to disclose limited data sets (i.e., data sets with no direct patient identifiers) for research and public health purposes if such an agreement exists.

\section{Sampling errors}

The standard error is primarily a measure of the sampling variability that occurs by chance when only a sample, rather than an entire universe, is surveyed. The standard error also reflects part of the measurement error but does not measure any systematic biases in the data. The chances are 95 out of 100 that an estimate from the sample differs from the value that would be obtained from a complete census by less than twice the standard error. The relative standard error (RSE) of an estimate is obtained by dividing the standard error by the estimate itself. The result is then expressed as a percent of the estimate.

The standard errors presented in the tables and used in tests of significance for this report were estimated using SUDAAN software. SUDAAN computes standard errors by using a first-order Taylor approximation of the deviation of estimates from their expected values. A description of the software and the approach it uses has been published (11). When it is not feasible to use statistical software such as SUDAAN or STATA for analyzing complex survey data, one may calculate approximate RSEs for aggregate estimates using generalized variance curve parameters that are described in Public-Use File Documentation (21). 


\section{Published and flagged estimates}

Estimates are not presented unless a reasonable assumption regarding their probability distributions is possible on the basis of the Central Limit Theorem. The Central Limit Theorem states that given a sufficiently large sample size, the sample estimate approximates the population estimate, and upon repeated sampling, its distribution would be approximately normal.

In this report, estimates are not presented if they are based on fewer than 30 cases in the sample data; only an asterisk (*) appears in the tables. Estimates based on 30 or more cases include an asterisk if the RSE of the estimate exceeds 30 percent.

\section{Estimation}

Statistics from the NHAMCS are derived by a multistage estimation procedure that produces essentially unbiased estimates. The estimation procedure has three basic components: inflation by reciprocals of the sampling selection probabilities, adjustment for nonresponse, and a population weighting ratio adjustment.

NHAMCS data were adjusted to account for two types of nonresponse. The first type occurred when a sample hospital refused to provide information about its ED that was publicly known to exist. In this case, the weights of visits to hospitals similar to the nonrespondent hospitals were inflated to account for visits represented by the nonrespondent hospitals. Hospitals were judged to be similar and grouped together for nonresponse purposes if they had the same ownership control group (voluntary nonprofit versus other) and region. Beginning with the 1998 data, formation of groups of similar hospitals also considered the MSA status of the hospital (in an MSA versus not in an MSA) with the following two exceptions: in the West, MSA status was not considered; and in non-MSA hospitals in the other three regions, ownership control group (voluntary nonprofit versus other) was not considered. This was done because the sample size was too small to use the finer breakdowns in the regions affected.
The second type of nonresponse occurred when a sample ESA within a responding hospital failed to provide completed Patient Record forms for a sample of patient visits. The weights of visits from responding ESAs were inflated to account for visits to similar nonresponding ESAs where ESAs were judged to be similar if they were in the same region. In the Northeast, Midwest, and South, ESA similarity also required having the same MSA status and in MSAs it also required being in the same ownership control group (voluntary nonprofit compared with other). In the West, ESA similarity required being in the same ownership control group (voluntary nonprofit compared with other). Starting in 2001, ESAs that responded minimally (i.e., provided substantially fewer Patient Record forms than expected) were considered nonrespondents for response rate calculations, but their records were included in the final data set. However, their total visit weights were set not to exceed 50 percent of the ESAs count of visits. The remaining weight for these minimally responding ESAs was accounted for by in-scope, responding ESAs of similar hospitals that were in the same PSU.

\section{Nonsampling errors}

As in any survey, results are subject to both sampling and nonsampling errors. Nonsampling errors include reporting and processing errors, as well as biases resulting from nonresponse and incomplete response. The magnitude of the nonsampling errors cannot be computed. However, these errors were kept to a minimum by procedures built into the operation of the survey. To eliminate ambiguities and encourage uniform reporting, attention was given to the phrasing of questions, terms, and definitions. Also, pretesting of most data items and survey procedures was performed. Quality control procedures and consistency and edit checks reduced errors in data coding and processing. Coding error rates ranged between 0.0 and 0.8 percent for various survey items.

Item nonresponse rates in the NHAMCS are generally low (5 percent or less). However, levels of nonresponse can vary considerably in the survey. Most nonresponse occurs when the needed information is not available in the medical record or is unknown to the person filling out the survey instrument. Nonresponse can also result when the information is available, but survey procedures are not followed and the item is left blank. In this report, the tables include a combined entry of "unknown or blank" to display missing data. For items where combined item nonresponse is between 30-50 percent, percent distributions are not discussed in the text. However, the information is shown in the tables. These data should be interpreted with caution. If nonresponse is random, the observed distribution for the reported item (i.e., excluding cases for which the information is unknown) would be close to the true distribution. However, if nonresponse is not random, the observed distribution could vary significantly from the actual distribution. Researchers need to decide how best to treat items with high levels of missing responses. For items with nonresponse greater than 50 percent, data are not presented.

Weighted item nonresponse rates (i.e., if the item was left blank or the unknown box was marked) were 5.0 percent or less for data items with the following exceptions: ethnicity (16.6 percent), reside in a nursing home or other institution (5.3 percent), mode of arrival (5.4 percent), primary expected source of payment (6.5 percent), waiting time (17.6 percent), duration (9.4 percent), temperature (7.8 percent), pulse (7.7 percent), systolic blood pressure (14.9 percent), diastolic blood pressure (15.1 percent), alcohol-related visits (11.8 percent), work-related visits (6.7 percent), seen in this ED within the last 72 hours (8.0 percent), episode of care (5.6 percent), intentionality of injury (17.0 percent of injury visits), cause of injury (15.7 percent of injury visits), and procedures (6.7 percent).

For some items, missing values were imputed by randomly assigning a value from Patient Record forms with similar characteristics. For the variable "immediacy with which patient should be seen" ( 2.7 percent with missing values, i.e., none of the categories were 
checked), the grouping was based on ED volume, geographic region, and three-digit ICD-9-CM code for primary diagnosis. The other imputed items were: birth year (3.1 percent), sex (0.4 percent), and race (12.0 percent). Imputation for these items was based on ED volume, geographic region, immediacy with which patient should be seen, and three-digit ICD-9-CM code for primary diagnosis. This represents a change from previous survey years when imputations were also performed for the following variables-ethnicity, disposition, and providers seen. Beginning in 1997, these latter items were no longer imputed. Blank or otherwise missing responses are noted in the data.

\section{Tests of significance and rounding}

In this report, the determination of statistical inference is based on the two-tailed $t$-test. The Bonferroni inequality was used to establish the critical value for statistically significant differences (0.05 level of significance) based on the number of possible comparisons within a particular variable (or combination of variables) of interest. Terms relating to differences such as "greater than" or "less than" indicate that the difference is statistically significant. A lack of comment regarding the difference between any two estimates does not mean that the difference was tested and found to be not significant.

In the tables, estimates of ED visits have been rounded to the nearest thousand. Consequently, estimates will not always add to totals. Rates and percents were calculated from original unrounded figures and do not necessarily agree with figures calculated from rounded data.

\section{Race and ethnicity}

The instruction for the race item on the Patient Record form was changed in 1999 to be consistent with standards issued by the Office of Management and Budget to promote comparability of data among Federal data sources. Among the standards issued in 1997 is the reporting of more than one race per patient (22).
The new race item includes the following groups: white, black or African American, Asian, Native Hawaiian or other Pacific Islander, and American Indian or Alaska Native. Respondents could check multiple categories for each patient. Prior to 1999, only a single race category could be checked per person. Estimates for specific race categories reflect visits where only a single race was reported. See "Population figures and rate calculation" in the "Technical Notes" for more information.

According to the same standards, race and Hispanic or Latino origin were collected separately. Consequently, all race categories include visits by persons of Hispanic or Latino and not Hispanic or Latino origin. Persons of Hispanic or Latino origin may be of any race.

\section{Calculation of time waiting to see a physician}

The NHAMCS collects data on the time the patient arrived in the ED and the time that the patient was seen by a physician. These two items were used to derive the amount of time spent waiting to see a physician. Waiting times longer than 12 hours were altered to assume that the a.m. or p.m. checkbox was completed incorrectly and that the patient did not actually wait that long. For visits where a physician was seen, 17.6 percent were missing the data needed to calculate waiting time.

\section{Calculation of time spent in the ED}

The NHAMCS collects data on the time the patient arrived in the ED and the time that the patient was discharged. If the discharge time was more than 24 hours from the arrival time, the hospital staff was asked to mark a checkbox. These three items were used to derive the amount of time the patient spent in the ED. At 9.4 percent of visits, the data needed to calculate time spent in the ED were missing.

\section{Injury groupings}

Table 13 presents data on the intent and mechanism producing the injuries that resulted in visits to EDs. Cause of injury is collected for each sampled injury visit in the NHAMCS and is coded according to the ICD-9-CM's "Supplementary Classification of External Causes of Injury and Poisoning." However, for table 13, the first-listed cause-of-injury data were regrouped to highlight the interaction between intentionality of the injury and the mechanism that produced the injury. Table I shows the E-code groupings used to produce this table. For table 14, the Barell Injury Diagnosis Matrix: Classification by Region of Body and Nature of Injury was used to show the distribution of injury-related visits by body site of primary diagnosis (18). Table II shows the ICD-9-CM groupings used to produce this table.

\section{Population figures and rate calculation}

The denominators used in calculating 2003 visit rates for age, sex, race, and geographic region are Census 2000-based postcensal estimates of the civilian noninstitutional population of the United States, with the exception of the text box, where the civilian institutionalized population was also used. The population estimates are special tabulations developed by the Population Division, U.S. Census Bureau, from the July 1, 2003, set of State population estimates by age, sex, and race. Population estimates of MSA status are based on data from the 2003 National Health Interview Survey (NHIS), National Center for Health Statistics, adjusted to the U.S. Census Bureau definition of core-based statistical areas as of December 2003. See www.census.gov/population/www/ estimates/metrodef.html for more about MSA definitions.

Estimates of visit rates for MSAs and non-MSAs in 2003 may differ somewhat from those reported in 2002 and previous years because of methodological differences in how the denominators were calculated. In survey years 1992-2002, the NHIS used a 1992 definition of MSAs and non-MSAs. The NHIS also used 1990-based census estimates as controls for calculating population estimates through 2002 . Because the NHAMCS used Census 
Table I. Reclassification of cause-of-injury codes for use with National Hospital Ambulatory Medical Care Survey data

\begin{tabular}{|c|c|}
\hline Intent and mechanism of injury & Cause-of-injury code ${ }^{1}$ \\
\hline 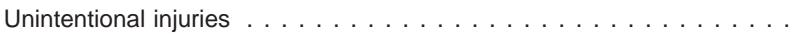 & E800-E848, E850-E869, E880-E929 \\
\hline 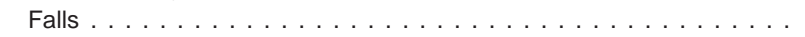 & E880.0-E886.9, E888 \\
\hline 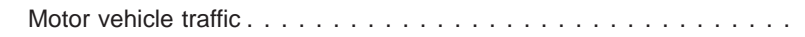 & E810-E819 \\
\hline Struck against or struck accidentally by objects or persons $\ldots \ldots \ldots$ & E916-E917 \\
\hline Overexertion and strenuous movements $\ldots \ldots \ldots \ldots$ & E927 \\
\hline Cutting or piercing instruments or objects $\ldots \ldots \ldots \ldots \ldots$ & E920 \\
\hline Natural and environmental factors $\ldots \ldots \ldots \ldots \ldots$ & E900-E909, E928.0-E928.2 \\
\hline $\begin{array}{l}\text { Poisoning by drugs, medicinal substances, biologicals, other solid and } \\
\text { liquid substances, gases, and vapors } \ldots \ldots \ldots \ldots \ldots \ldots\end{array}$ & E850-E869 \\
\hline $\begin{array}{l}\text { Fire and flames, hot substance or object, caustic or corrosive } \\
\text { material, and steam } \ldots \ldots \ldots \ldots \ldots \ldots \ldots \ldots \ldots \ldots \ldots \ldots \ldots \ldots \ldots\end{array}$ & E890-E899, E924 \\
\hline Machinery . . . . . . . . . . . . . . . . . . . . & E919 \\
\hline 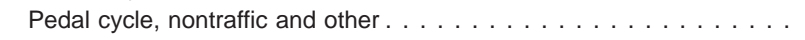 & E800-E807(.3), E820-E825(.6), E826.1, E826.9 \\
\hline Motor vehicle, nontraffic . . . . . . . . . . . . . . . . . & E820-E825(.0 -.5,. .7-.9) \\
\hline Other transportation. . . . . . . . . . . . . . . . . . & E800-E807(. -.2,. 8-.9), E826(.0,. 2-.8), E827-E829, E831, E833-E845 \\
\hline 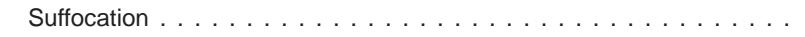 & E911-E913 \\
\hline Firearm missile. . . . . . . . . . . . . . . . . . . . . & E922 \\
\hline Drowning or submersion $\ldots \ldots \ldots \ldots \ldots \ldots \ldots$ & E830, E832, E910 \\
\hline Foreign body . . . . . . . . . . . . . . . . . . . & E914-E915 \\
\hline Caught accidentally in or between objects $\ldots \ldots \ldots \ldots$ & E918 \\
\hline Other and not elsewhere classified $\ldots \ldots \ldots \ldots$ & E846-E848, E921, E923, E925-E926, E928.3, E928.8, E929.0-E929.5 \\
\hline 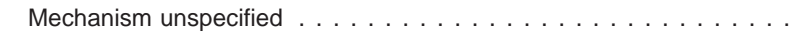 & E887, E928.9, E929.8, E929.9 \\
\hline 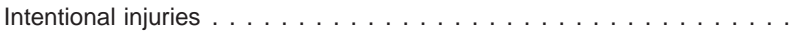 & E950-E959, E960-E969, E970-E978, E990-E999 \\
\hline 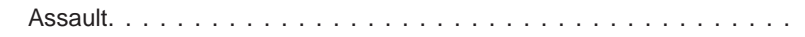 & E960-E969 \\
\hline Unarmed fight or brawl, striking by blunt or thrown object . . . . . . . & E960.0, E968.2 \\
\hline Cutting or piercing instrument $\ldots \ldots \ldots \ldots \ldots$ & E966 \\
\hline 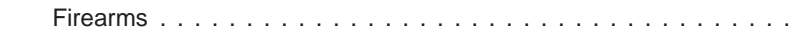 & E965.0-E965.4 \\
\hline Other and unspecified mechanism $\ldots \ldots \ldots \ldots \ldots$ & E960.1, E961-E964, E965.5-E965.9, E967-E968.1, E968.3-E969 \\
\hline Self-inflicted . . . . . . . . . . . . . . . . . . & E950-E959 \\
\hline Poisoning by solid or liquid substances, gases, and vapors . . . . . . & E950-E952 \\
\hline Cutting and piercing instrument $\ldots \ldots \ldots \ldots \ldots$ & E956 \\
\hline Suffocation. . . . . . . . . . . . . . . . . . & E953 \\
\hline Other and unspecified mechanism $\ldots \ldots \ldots \ldots \ldots$ & E954-E955, E957-E959 \\
\hline Other causes of violence $\ldots \ldots \ldots \ldots \ldots \ldots$ & E970-E979, E990-E999 \\
\hline Injuries of undetermined intent $\ldots \ldots \ldots \ldots \ldots \ldots \ldots$ & E980-E989 \\
\hline Adverse effects of medical treatment. . . . . . . . . . . . & E870-E879, E930-E949 \\
\hline
\end{tabular}

${ }^{1}$ Based on the "Supplementary Classification of External Causes of Injury and Poisoning," International Classification of Diseases, 9th Revision, Clinical Modification (ICD-9-CM) (17).

2000-based estimates beginning in 2001, adjustments needed to be made to the MSA figures obtained from the NHIS in 2001 and 2002. For 2003, special tabulations were obtained from the Office of Analysis and Epidemiology, NCHS, where 2003 NHIS data were matched to the December 2003 U.S. Census Bureau definition of core-based statistical areas. The estimates were further adjusted based on the 2003 population estimates obtained from the Census Bureau.

Denominators used in computing estimates of visit rates by expected source of payment were obtained from the 2003 NHIS. Individuals reporting multiple insurance categories in the NHIS were counted in each category they reported with the exception of Medicaid and SCHIP, which were combined into a single category.
Population estimates for race groups in the 2003 NAMCS and NHAMCS are based on Census 2000, in which respondents were able to indicate more than one race category (as requested by the 1997 Standards for Federal Data on Race and Ethnicity) (22). Starting with 2001, the denominators used for calculating race-specific visit rates reflect the transition to multiple-race reporting. Specific race denominators reflect persons with a single race identification, and a separate denominator is available for persons of multiple races. In this report, a visit rate for white persons, for example, uses a denominator that reflects the "white only" population, and the numerator is the number of visits in which white and no other race category was reported as the patient's race by the health care provider.
Data indicate that multiple races are recorded less frequently in medical records than occur in the general population. The 2003 population estimates indicate that multiple-race persons account for 1.5 percent of the total population whereas multiple-race patients (as indicated by the provider) account for 0.1 percent of ED visits. This difference exists because hospital staff is less likely to know and record the multiple race preference of the patient and not because, after ageadjusting, persons with multiple races make fewer ED visits. This implies that the race population rates calculated in 2003 are probably slight "overestimates" for the single race categories and "underestimates" for the multiple race category. 
Table II. Reclassification of body site of primary diagnosis codes for use with the National Hospital Ambulatory Medical Care Survey emergency department data

\begin{tabular}{|c|c|}
\hline Body site & ICD-9-CM codes $^{1}$ \\
\hline \multicolumn{2}{|l|}{ Head and neck: } \\
\hline Traumatic brain injury. . . . . . . . . . . . . . . . . & $\begin{array}{l}800.1-800.4,800.6-800.9,800.03-800.05,800.50-800.55,801.1-801.4,801.6-801.9,801.03-801.05, \\
801.50-801.55,803.1-803.4,803.6-803.9,803.03-803.05,803.50-803.55,804.1-804.4804 .6-804.9, \\
804.03-804.05,804.50-804.55,850.2-850.4,851-853,854.03-854.05,854.1,950.1-950.3,800.00-800.02, \\
800.06,800.09,801.00-801.02,801.06,801.09,803.00-803.02,803.06,803.09,804.00-804.02,804.06, \\
804.09,800.50-800.52,800.56,800.59,801.50-801.52,801.56,801.59,803.50-803.52,803.56,803.59, \\
804.50-804.52,804.56,804.59,850.0,850.1,850.5,850.9,854.00-854.02,854.06,854.09, \text { or } 995.55\end{array}$ \\
\hline Other head $\ldots \ldots \ldots \ldots \ldots \ldots \ldots \ldots$ & $951,873.0,873.1,873.8,873.9,941 . x 6$, or 959.01 \\
\hline Face .................... & $802,830,848.0-848.1,872,873.2-873.7,941 . x 1,941 . x 3-941 . x 5$, or 941.x7 \\
\hline Eye. . . . . . . . . . . . . . . . & $950.0,950.9,870-871,921,918,940$, or $941 . x 2$ \\
\hline Head, face, neck, and unspecified. . . . . . . . . . . & $\begin{array}{l}807.5-807.6,848.2,925.2,953.0,954.0,874,941 . \times 8,925.1,900,957.0,910,920,947.0,959.09,941 . \times 0 \\
\text { or } 941 . x 9\end{array}$ \\
\hline Spinal cord $\ldots \ldots \ldots \ldots \ldots \ldots \ldots \ldots$ & $\begin{array}{l}\text { 806.0-806.1, 952.0, 806.2-806.3, 952.1, 806.4-806.5, 952.2, 806.6-806.7, 952.3-952.4, 806.8-806.9, } \\
\text { 952.8-952.9, 957.0, or 959.09 }\end{array}$ \\
\hline \multicolumn{2}{|l|}{ Vertebral column . . . . . . . . . . . . . . . . . . . } \\
\hline Cervical . . . . . . . . . . . . . . . & $805.0-805.1,839.0-8391$, or 847.0 \\
\hline Thoracic and dorsal. . . . . . . . . . . . . . . . . . & $805.2-805.3,839.21,839.31$, or 847.1 \\
\hline Lumbar . . . . . . . . . . . . . . . . . . . . & $805.4-805.5,839.20,839.30$, or 847.2 \\
\hline Other vertebral column . . . . . . . . . . . . . . & $\begin{array}{l}805.6-805.7,839.41,839.42,839.51-839.52,847.3-847.4,805.8-805.9,839.40,839.49 \\
839.50, \text { or } 839.59\end{array}$ \\
\hline \multicolumn{2}{|l|}{ Torso: } \\
\hline Chest . . . . . . . . . . . . . . . & $\begin{array}{l}\text { 807.0-807.4, 839.61, 839.71, 848.3-848.4, 926.19, 860-862, 901, 953.1, 875, 879.0, 879.1, 922.0, 922.1, } \\
\text { 922.33, or 942.x1-942.x2 }\end{array}$ \\
\hline Abdomen . . . . . . . . . . . . . . . . & $863-866,868,902.0-902.4,953.2,953.5,879.2-879.5,922.2,942 . x 3$, or 947.3 \\
\hline Pelvis and urogenital $\ldots \ldots \ldots \ldots \ldots$ & $\begin{array}{l}808,839.69,839.79,846,848.5,926.0,926.12,867,902.5,902.81-902.82,953.3,877-878,922.4,942 . x 5 \text {, } \\
\text { or } 947.4\end{array}$ \\
\hline Trunk. . . . . . . . . . . . . . . . . . . . . . & 809, 926.8-926.9, 954.1, 954.8-954.9, 879.6-879.7, 922.8-922.9, 911, 942.0, 942.9, or 959.1 \\
\hline Back and buttocks. . . . . . . . . . . . . . . . & $847.9,926.11,876,922.32,922.31$, or $942 . x 4$ \\
\hline \multicolumn{2}{|l|}{ Upper extremity: } \\
\hline Shoulder and upper arm . . . . . . . . . . . . . . . & $810-812,831,840,880,887.2-887.3,943 . x 3-943 . x 6,912,923.0,927.0$, or 959.2 \\
\hline Forearm and elbow . . . . . . . . . . . . . . & $813,832,841,881 . x 0-881 . x 1,887.0-887.1,923.1,927.1$, or 943.x1-943.x2 \\
\hline Wrist, hand, and fingers $\ldots \ldots \ldots \ldots \ldots$ & $\begin{array}{l}\text { 814-817, 833-834, 842, 881.x2, 882-883, 885-886, 914-915, 923.2-923.3, 927.2-927.3, 944, } \\
\text { or 959.4-959.5 }\end{array}$ \\
\hline Other and unspecified upper extremity . . . . . . . . . & $818,884,887.4-887.7,903,913,959.3,923.8-923.9,927.8-927.9,953.4,955$, or 943.x0-943.x9 \\
\hline \multicolumn{2}{|l|}{ Lower extremity: } \\
\hline Нір . . . . . . . . . . . . . . . . & $820,835,843,924.01,928.01$ \\
\hline Upper leg and thigh. . . . . . . . . . . . . & $821,897.2-897.3,924.00,928.00$, or $945 . x 6$ \\
\hline 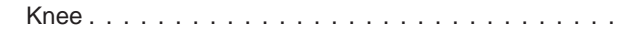 & $822,836,844.0-844.3,924.11,928.11$, or $945 . \times 5$ \\
\hline Lower leg and ankle . . . . . . . . . . . . . . & $823-824,897.0-897.1,837,845.0,924.10,924.21,928.10,928.21$, or $945 . x 3-945 . x 4$ \\
\hline Foot and toes . . . . . . . . . . . . . & 825-826, 838, 845.1, 892-893, 895-896, 917, 924.20, 924.3, 928.20, 928.3, or 945.x1-945.x2 \\
\hline Other and unspecified lower extremity $\ldots \ldots \ldots \ldots$ & $\begin{array}{l}\text { 827, 844.8-844.9, 890-891, 894, 897.4-897.7, 904.0-904.8, 916, 924.4-924.5, 928.8, 928.9, } \\
\text { 959.6-959.7, 945.x0, or 945.x9 }\end{array}$ \\
\hline System-wide . . . . . . . . . . . . . . . . & $\begin{array}{l}\text { 930-939, 960-994, 905-908, 909.0, 909.1, 909.2, 909.4, 909.9, 958, 995.80-995.85, 995.50-995.54, } \\
\text { 995.80, 995.85, 909.0, 909.1, 909.2, 909.4, 909.9 }\end{array}$ \\
\hline Other and unspecified body site injuries. . . . . . . . . & $\begin{array}{l}828,819,902.87,902.89,953.8,947.1-947.2,956,829,839.8-839.9,848.8-848.9,869,879.8-879.9, \\
902.9,904.9,919,924.8-924.9,929,946,947.8-947.9,948-949,953.9,957.1,957.8-957.9 \text { or } 959.8-959.9\end{array}$ \\
\hline Adverse effects and medical complications . . . . . . . . . & $909.3-909.5,995.0-995.4,995.6,995.86-995.89,996-999$ \\
\hline
\end{tabular}

${ }^{1}$ Based on the "Supplementary Classification of External Causes of Injury and Poisoning," International Classification of Diseases, 9th Revision, Clinical Modification (ICD-9-CM) (17).

\section{Definition of terms}

Drug mention-A drug mention is the health care provider's entry on the Patient Record form of a pharmaceutical agent - by any route of administration - for prevention, diagnosis, or treatment. Generic as well as brandname drugs are included as are nonprescription and prescription drugs. Along with all new drugs, the physician also records continued medications if the patient was specifically instructed during the visit to continue the medication. Health care providers may report up to six medications per visit.

Drug visit-A drug visit is a visit at which medication was prescribed or provided by the physician.

Emergency department-An ED is a hospital facility for the provision of unscheduled outpatient services to patients whose conditions require immediate care and is staffed 24 hours a day. If an ED provided emergency services in different areas of the hospital, then all of these ESAs are selected with certainty into the sample.
Off-site EDs that are open less than 24 hours are included if staffed by the hospital's ED.

Emergency service area-An emergency service area is the smallest administrative unit of an ED where separate patient statistics are kept. It may be located on hospital grounds or operated off site by the hospital.

Emergent visit-An emergent visit is one at which the triage practitioner determines that the patient should receive care immediately to combat danger to life or limb and where any 
delay would likely result in deterioration. If the visit was determined to be emergent, "less than 15 minutes" was to be checked in item $2 b$, "immediacy with which patient should be seen" on the Patient Record form.

Episode of care-This term describes the nature of the care provided at the visit; an initial visit versus a followup visit. An episode of care begins with the initial visit for care for a particular problem and ends when the patient is no longer continuing treatment. A problem may recur later, but that is considered a new episode of care. An initial visit may be diagnostic in nature whereas a followup visit may be to check progress or continue therapy.

Followup visit-Care was previously provided for this problem. This is the second or subsequent visit for this problem or complaint.

Hospital-To be in scope for the NHAMCS, a hospital must have an average length of stay for all patients of less than 30 days (short-stay) or be a hospital whose specialty is general (medical or surgical) or children's general, except Federal hospitals, hospital units of institutions, and hospitals with fewer than six beds staffed for patient use.

Illness-related visit-A visit is considered illness related if it was not an injury visit, as in the definition for injury-related visit.

Initial visit-This is the first visit to this ED by this patient for care of this particular problem or complaint.

Injury-related visit-A visit is injury related if "Yes" was checked in response to item 5a, "Is visit related to injury, or poisoning, or adverse effect of medical treatment?;" if a cause of injury or a nature of injury diagnosis was provided; or if an injury-related reason for visit was reported.

\section{Outpatient department-An} outpatient department is a hospital facility where nonurgent ambulatory medical care is provided under the supervision of a physician.

Ownership-Hospitals are designated according to the primary owner of the hospital based on the Verispan Hospital Database.
Voluntary nonprofit-Hospitals are church-related, a nonprofit corporation, or have other nonprofit ownership.

Government, non-FederalHospitals are operated by a State, county, city, city-county, or hospital district or authority.

Proprietary-Hospitals are individually or privately owned or are partnerships or corporations.

Patient_A patient is an individual seeking personal health services who is not currently admitted to any health care institution on the premises. Patients arriving by ambulance are included.

Visit-A visit is a direct, personal exchange between an ambulatory patient seeking care and a physician or other hospital staff member working under the physician's supervision for the purpose of rendering personal health services. Excluded from the NHAMCS are visits where medical care was not provided, such as visits made to drop off specimens, pay bills, and make appointments.

Visit rate-The visit rate is a basic measure of service utilization for event-based surveys. The numerator is the number of estimated visits and the denominator is the corresponding U.S. population estimate for those who possibly could have made the visits. The interpretation is that for every person in the population there are $x$ visits made. It does not mean that $x$ percent of the population made visits because some persons in the population make no visits and others make multiple visits within a given year. The only exception is when an event can occur just once for a person (e.g., if an appendectomy were performed during the visit). The visit rate is best used to compare amounts of utilization across various subgroups of interest such as age, race, or geographic region (e.g., the rate of U.S. emergency department visits in 2003 was 69.3 visits per 100 black or African American persons and 37.2 visits per 100 white persons). 


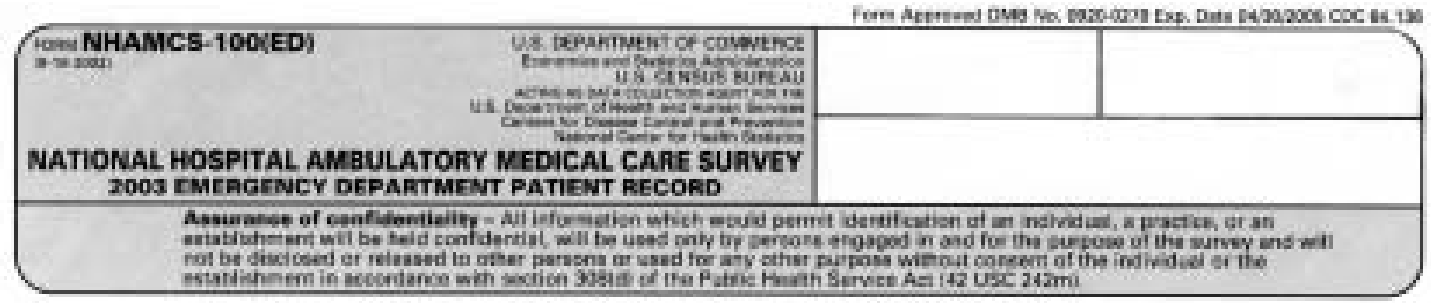

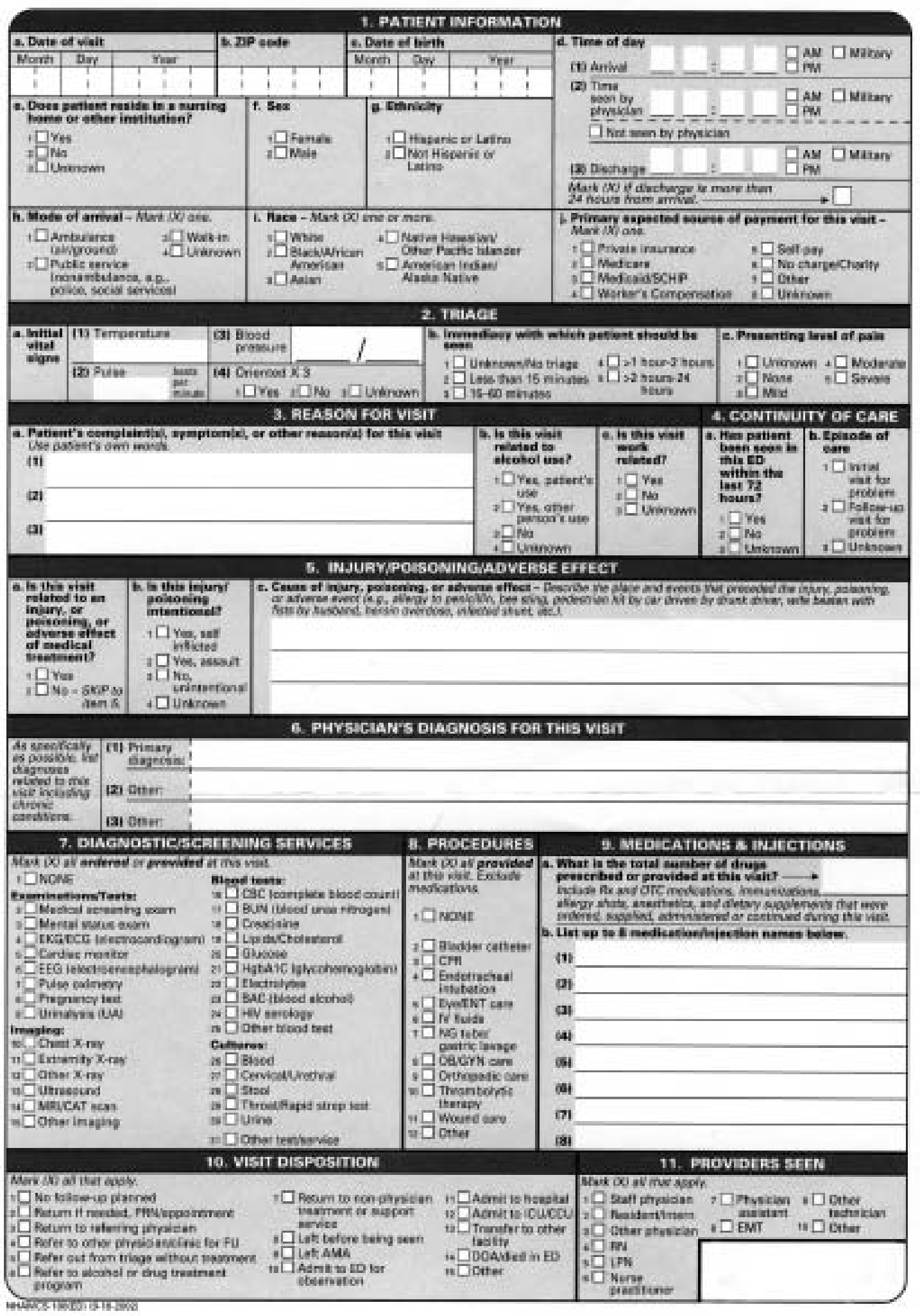

Figure I. Patient Record form 


\section{Suggested citation}

McCaig LF, Burt CW. National Hospital Ambulatory Medical Care Survey: 2003 emergency department summary. Advance data from vital and health statistics; no 358. Hyattsville, Maryland: National Center for Health Statistics. 2005.

\section{Copyright information}

All material appearing in this report is in the public domain and may be reproduced or copied without permission; citation as to source, however, is appreciated.
National Center for Health Statistics

Director

Edward J. Sondik, Ph.D.

Acting Co-Deputy Director Jennifer H. Madans, Ph.D.

Acting Co-Deputy Director Michael H. Sadagursky

\section{U.S. DEPARTMENT OF HEALTH \& HUMAN SERVICES}

Centers for Disease Control and Prevention National Center for Health Statistics 3311 Toledo Road

Hyattsville, Maryland 20782

OFFICIAL BUSINESS

PENALTY FOR PRIVATE USE, $\$ 300$

To receive this publication regularly, contact the National Center for Health Statistics by calling 1-866-441-NCHS (6247)

E-mail: nchsquery@cdc.gov

Internet: www.cdc.gov/nchs

DHHS Publication No. (PHS) 2005-1250 05-0280 (05/05)

\section{Trade name disclaimer}

The use of trade names is for identification only and does not imply endorsement by the Centers for Disease Control and Prevention, U.S. Department of Health and Human Services. 